\title{
Activation patterns in Atrial Fibrillation: Contributions of Body Surface Potential Mapping
}

María de la Salud Guillem Sánchez

Supervisors:

Dr. José Millet

Dr. Francisco Castells

December, 2008 




\section{Agradecimientos}

Quiero expresar mi agradecimiento a todas las personas que me han apoyado durante la realización de esta tesis doctoral y que han sido muchas.

A mi madre y a mi tía, que no sólo me han apoyado anímicamente durante la realización de esta tesis, sino que sin su ayuda, aguja e hilo en mano, sus ideas y su trabajo ni siquiera hubiera podido comenzar a desarrollar esta tesis.

A mis directores de tesis Pepe Millet y Paco Castells. A Pepe quiero agradecerle la confianza que siempre ha depositado en mí y el haberme apoyado siempre en todo lo que le ha sido posible durante estos años. A Paco quiero agradecerle su ayuda durante la última etapa de la realización de la tesis y especialmente su meticulosidad en la redacción científica que tanto ha mejorado la calidad de mi trabajo.

A los doctores Javier Chorro y Vicent Bodí del Hospital Clínico Universitario de Valencia. Ellos fueron los primeros en dedicarme su tiempo y permitirme tomar registros en su consulta con la ayuda de los entonces residentes Mauricio Pellicer y Lorena Peiró.

Al doctor Steven Swiryn y al profesor Alan Sahakian por haberme permitido trabajar con ellos en su grupo en la Northwestern University en EEUU. Ellos supieron transmitirme su dinamismo, su entrega a la investigación y de ellos he aprendido cómo poner en práctica el método científico desde la planificación del trabajo hasta la presentación de resultados.

$\mathrm{Al}$ doctor Andreas Bollmann y a la doctora Daniela Husser del Hospital de Leipzig en Alemania por haber creído en mi proyecto y haberse involucrado activamente en mi investigación desde la toma registros a los pacientes de su hospital al diseño de los estudios o colaboración en la difusión de resultados.

Al doctor Aurelio Quesada del Hospital General de Valencia con quien estamos colaborando actualmente poniendo en práctica los métodos desarrollados en esta tesis. Con él, además de llevar a cabo proyectos muy prometedores, es todo un placer colaborar gracias a su inquietud científica y a su calidad humana.

Al doctor Ramón Brugada, con el que he tenido el honor poder colaborar y con el que espero poder tener el honor de seguir colaborando en el futuro. Con él no sólo estoy teniendo la posibilidad de realizar investigación puntera en el campo de la cardiología, sino que también he tenido la oportunidad de conocer 
a un científico ejemplar y una persona excepcional.

A los pacientes y voluntarios que han participado en los estudios que han dado lugar a esta tesis y que han tenido que soportar con paciencia que les llenara el cuerpo de cables y cintas de una forma totalmente desinteresada. Sin ellos no me hubiera sido posible desarrollar esta tesis.

A los que han sido mis compañeros de laboratorio desde que empecé a interesarme por la investigación durante la realización de mi proyecto final de carrera y que tanto me animaron en los momentos difíciles: Fili, Pepelu, Natalia, Diego, Cibeles, Robin, Vicente, David, Laura, José Antonio y también a todos los que han formado parte del grupo de investigación durante todos estos an̄os, y más especialmente a mis compan̄eros actuales: Vicky, Eduard, Xavi, Lola, Lorena y María. Tampoco quiero olvidarme de Jose Antonio Pérez, técnico de la escuela de Telecomunicación que me ha ayudado desinteresadamente durante todo este tiempo.

A todos los que han tenido que sufrir mis discursos sobre elecrocardiografía, fibrilación auricular, congresos, artículos, etc. quizá más de lo que hubiera sido necesario, y especialmente a Pablo que no sólo me ha escuchado y apoyado sino que fue él quien me animó a entrar en el mundo de la investigación y me ha acompañado siempre en los buenos y los malos momentos.

A Andreu, porque ha aguantado mis quejas y celebrado mis logros como si fueran suyos. Porque me animó a seguir adelante cuando ya casi lo daba todo por perdido y gracias a compartir conmigo sus ideas y a discutirme las mías al final todo cobró forma y puedo ahora presentar esta tesis. Porque esta tesis es también suya. 


\section{Acronyms}

$\begin{array}{ll}\text { AMI } & \text { Acute Myocardial Infarction } \\ \text { AF } & \text { Atrial Fibrillation } \\ \text { AFL } & \text { Atrial Flutter } \\ \text { AV } & \text { Atrioventricular } \\ \text { BBB } & \text { Bundle Branch Block } \\ \text { BSPM } & \text { Body Surface Potential Mapping } \\ \text { DACL } & \text { Dominant Atrial Cycle Length } \\ \text { ECG } & \text { Electrocardiogram } \\ \text { EGM } & \text { Electrogram } \\ \text { FAA } & \text { Dominant frequency corresponding to Atrial Activity } \\ \text { FFT } & \text { Fast Fourier Transform } \\ \text { HCUV } & \text { Hospital Clínico Universitario de Valencia } \\ \text { LHC } & \text { Leipzig Heart Center } \\ \text { LSV } & \text { Least Squared Value } \\ \text { MDL } & \text { Minimum Description Length } \\ \text { MI } & \text { Myocardial Infarction } \\ \text { OVGUH } & \text { Otto-von-Guericke University Hospital } \\ \text { PCA } & \text { Principal Component Analysis } \\ \text { RMS } & \text { Root Mean Squared } \\ \text { SA } & \text { Sinoauricular } \\ \text { SVD } & \text { Singular Value Decomposition } \\ \text { VCG } & \text { Vectorcardiogram } \\ \text { VH } & \text { Ventricular Hypertrophy } \\ \text { WCT } & \text { Wilson Central Terminal }\end{array}$





\section{Contents}

Agradecimientos iii

Acronyms $\quad$ v

$\begin{array}{lll}\text { Sumari } & \text { XV }\end{array}$

Sumario $\quad$ xvii

Abstract $\quad$ xix

1 Introduction 1

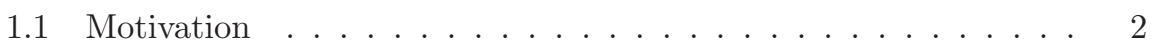

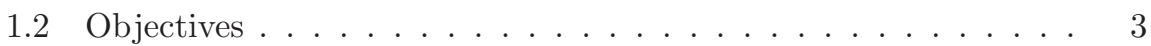

1.3 Structure of the thesis . . . . . . . . . . . . . . 4

2 State of the art $\quad 7$

2.1 Introduction to Electrocardiography . . . . . . . . . . . 8

2.1.1 The heart . . . . . . . . . . . . . 8

2.1.2 The surface electrocardiogram . . . . . . . . . . . 9

2.1.3 The vectorcardiogram ... . . . . . . . . . . 13

2.1.4 Body Surface Potential Mapping . . . . . . . . . . . . 16

2.2 Atrial fibrillation . . . . . . . . . . . . . . . . . 18

2.2.1 Pathophysiology . . . . . . . . . . . . . 18

2.2 .2 Epidemiology . . . . . . . . . . . . . . . 20

2.2.3 Treatment of atrial fibrillation . . . . . . . . . . . 20

2.3 Spatial characterization of ECG signals . . . . . . . . . . . . 21

2.3.1 Derivation of orthogonal leads from the standard electrocardiogram . . . . . . . . . . . . . 21

2.3.2 Study of spatial characteristics of derived atrial loops during atrial fibrillation . . . . . . . . . . . . . . . 23

2.4 Optimization of electrocardiographic leads . . . . . . . . . . . 24

2.4.1 Determination of the number and location of optimum electrocardiographic leads . . . . . . . . . . . . 24 
2.4.2 Repositioning of electrocardiographic leads for the study of atrial fibrilation . . . . . . . . . . . . . . 28

2.5 Organization degree of atrial fibrillation . . . . . . . . . 30

2.5.1 Quantification of the temporal organization of AF . . . 30

2.5.2 Quantification of the spatial organization of AF . . . . . 32

2.5.3 Understanding the mechanisms of atrial fibrillation . . . . 33

2.5.4 Clinical implications .............. 35

3 Materials $\quad 37$

3.1 PTB database . . . . . . . . . . . . . . . . . . . . . . . . . . . . 37

3.2 BSPM recordings . . . . . . . . . . . . . . . . . . . . . . . . . . . . . . . 38

3.2 .1 Acquisition system . . . . . . . . . . . . . . . . . . 38

3.2.2 Study Population . . . . . . . . . . . . . . . . 41

4 Spatial characteristics from the ECG $\quad 47$

4.1 Introduction . . . . . . . . . . . . . . . . . . . 48

4.2 Materials and Methods . . . . . . . . . . . . . . . . 50

4.2.1 Study Population . . . . . . . . . . . . . . . 50

4.2.2 Signal conditioning . . . . . . . . . . . . . . . . . 50

4.2.3 Preprocessing of $\mathrm{P}$ waves .............. . . . . . . 51

4.2.4 Preprocessing of AF waves . . . . . . . . . . . . 52

4.2.5 Optimized transforms for P-wave and QRS complex derivation .................. 52

4.2.6 Stability of transform matrices . . . . . . . . . . . 53

4.2.7 Derivation of orthogonal leads from the ECG . . . . . . 53

4.2.8 Performance on the $\mathrm{P}$ wave and QRS complex . . . . . 53

4.2 .9 Performance on AF waves . . . . . . . . . . . . . . . 54

4.3 Results . . . . . . . . . . . . . . . . . 56

4.3.1 Transform matrices . . . . . . . . . . . . . 56

4.3.2 Stability of transform matrices . . . . . . . . . . 56

4.3.3 Comparison of true vs. derived $\mathrm{P}$ waves . . . . . . . . . 56

4.3.4 Comparison of true vs. derived AF waves . . . . . . . . 63

4.4 Discussion . . . . . . . . . . . . . . . . . 68

4.4.1 Main findings . . . . . . . . . . . . . . . . . 68

4.4.2 Transform matrix optimized for the $\mathrm{P}$ wave . . . . . . . 68

4.4.3 On the derivation of AF loops from the ECG . . . . . . . 69

4.4.4 Limitations of the study . . . . . . . . . . . . . 71

4.5 Conclusions .................... . . 71

5 Optimization of ECG leads $\quad 73$

5.1 Introduction . . . . . . . . . . . . . . . . . 74

5.2 Evaluation of lead selection methods . . . . . . . . . . . . 76

5.2.1 Materials and Methods ............. . . 76

5.2 .2 Results .................... 79 
5.2 .3 Discussion ................... . . 84

5.3 Selection of ECG leads for AF . . . . . . . . . . . . . . 88

5.3.1 Materials and Methods ............. 88

5.3 .2 Results .................... . . 92

5.3 .3 Discussion . . . . . . . . . . . . . . . . 99

5.4 Conclusions . . . . . . . . . . . . . . . . . . . . . 104

6 Non-invasive mapping of human AF 105

6.1 Introduction . . . . . . . . . . . . . . . 106

6.2 Materials and Methods . . . . . . . . . . . . . . . 107

6.2.1 Study Population . . . . . . . . . . . . . . 107

6.2.2 ECG Signal Processing . . . . . . . . . . . . 107

6.2.3 Map display and wavefront propagation descriptors . . . . 109

6.2.4 Assessment of short-term reproducibility . . . . . . . . . . 111

6.3 Results . . . . . . . . . . . . . . . . . . 111

6.3.1 Atrial fibrillatory signals on the body surface . . . . . . . 111

6.3.2 Surface activation patterns during AF . . . . . . . . . 113

6.3.3 Surface activation patterns during AF and similarity among AF signals on the body surface . . . . . . . . . 113

6.3.4 Short-term reproducibility of dominant activation patterns 115

6.4 Discussion . . . . . . . . . . . . . . . . . . . 117

6.4.1 Main findings . . . . . . . . . . . . . . . . 117

6.4.2 Comparison with invasive mapping data . . . . . . . . . 117

6.4.3 Comparison with non-invasive studies . . . . . . . . . . 118

6.4.4 Limitations . . . . . . . . . . . . . . . . . 118

6.5 Conclusions . . . . . . . . . . . . . . . . . . . 119

$\begin{array}{lll}7 & \text { Discussion and conclusion } & 121\end{array}$

7.1 Discussion . . . . . . . . . . . . . . . . . . 121

7.1 .1 Main findings . . . . . . . . . . . . . . . . 122

7.1.2 Comparison with previous studies . . . . . . . . . 123

7.1 .3 Limitations . . . . . . . . . . . . . . . . . . . . 125

7.2 Conclusion . . . . . . . . . . . . . . . 125

7.3 Guides for future work . . . . . . . . . . . . . . . . . 126

7.3.1 Spatiotemporal quantification of atrial organization . . . 126

7.3.2 Clinical applications . . . . . . . . . . . . . . . . 127

7.3.3 Transfer of technology . . . . . . . . . . . . . 127

8 Contributions $\quad 129$

8.1 Publications . . . . . . . . . . . . . . . . . 129

8.1.1 Main contributions of this thesis . . . . . . . . . . 129

8.1.2 Contributions related to this thesis . . . . . . . . . . 130

8.2 Framework of the Dissertation . . . . . . . . . . . . . 131

8.2.1 Research projects ................. 131 
8.2.2 International research stays . . . . . . . . . . . . . 133

8.2 .3 Collaborations . . . . . . . . . . . . . . . 133 


\section{List of Figures}

2.1 Anatomy of the heart, valves and vessels . . . . . . . . . . 8

2.2 Intrinsic conduction system of the heart . . . . . . . . . . . . . 10

2.3 The electric heart vector and its projection on limb leads . . . . 11

2.4 Standard positions of the ECG electrodes . . . . . . . . . . . . 11

2.5 Normal ECG tracing . . . . . . . . . . . . . . . . . . . . . 13

2.6 Electrode positions of Frank's vectorcardiographic system. . . . . 15

2.7 A normal vectorcardiogram . . . . . . . . . . . . . . 15

2.8 Example of isopotential maps . . . . . . . . . . . . . . . . 16

2.9 ECG tracings during sinus rhythm and atrial fibrillation . . . . . 18

2.10 Conceptual models of atrial fibrillation . . . . . . . . . . . . . . . 19

2.11 Vector loops of six consecutive AF waves . . . . . . . . . . . . . . 23

2.12 Adaptation of the standard 12-lead electrocardiogram . . . . . . 29

2.13 Time-frequency analysis of AF signals . . . . . . . . . . . . . 31

2.14 Mapping criteria for classification of the electrical organization degree in $\mathrm{AF}$. . . . . . . . . . . . . . . . . . . . . . . . 32

2.15 Isochrone maps and pseudo-EGs constructed from LA and RA optical recordings of an episode of AF . . . . . . . . . . . 34

3.1 Example of recording from PTB database . . . . . . . . . . . . . 39

3.2 Elements constituting our BSPM system . . . . . . . . . . . . . . 40

3.3 Electrode positioning of the BSPM system in configuration 1 . . 41

3.4 Sketches of the electrode support system for configuration 1 . . . 42

3.5 Arrangement of the electrodes and belt used for their attachment to the patient in configuration 2 . . . . . . . . . . . . . 43

3.6 Sketch of the electrode support system for configuration 2 . . . . 43

3.7 Software interface for the acquisition of BSPM recordings . . . . 44

3.8 Example of 64-lead recording of a healthy subject . . . . . . . . . 45

3.9 Example of 56-lead recording of a one patient with AF . . . . . . 46

4.1 True versus derived ECGs with Dower, PLSV and QLSV transforms of patient $2 \ldots \ldots . \ldots 57$ 
4.2 True versus derived VCGs corresponding to the $\mathrm{P}$ wave with Dower, PLSV and QLSV transforms of patient 2 . . . . . . . 58

4.3 True versus derived VCGs corresponding to the QRS complex with Dower, PLSV and QLSV transforms of patient 2 . . . . . 59

4.4 RMS error of derived versus true measured P-loops using Dower, PLSV and QLSV transforms . . . . . . . . . . . . . . 60

4.5 Root mean squared error of derived versus true measured $\mathrm{P}$ waves in the three orthogonal leads X,Y,Z using Dower, PLSV and QLSV transforms . . . . . . . . . . . . . . 61

4.6 Correlation of derived vs. true measured P-waves . . . . . . . . . 61

4.7 RMS error of derived versus true measured QRS-loops . . . . . . 63

4.8 Root mean squared error of derived versus true measured QRS complexes ...................... 64

4.9 Correlation of derived vs. true measured QRS complexes . . . . . 64

4.10 Examples of different AF loops and their fit to a plane . . . . . . 65

4.11 Histographic representation of the fit to the plane $\left(R^{2}\right)$ of AF waves 65

4.12 Franks versus derived orthogonal AF waves using Dower's inverse transform ..................... 66 66

4.13 Histographic representation of errors in reconstruction of derived waves with Dower's inverse transform . . . . . . . . . . 67

5.1 Leads selected for different datasets and selection methods . . . . 79

5.2 Error for limited lead sets on the whole ECG . . . . . . . . . . . 81

5.3 Correlation coefficients for limited lead sets on the whole ECG . 82

5.4 Error for limited lead sets on the whole ECG for the BBB group 84

5.5 Error for limited lead sets on the QRS complex for the BBB group 85

5.6 Error for limited lead sets on the $\mathrm{P}$ wave . . . . . . . . . . . . 85

5.7 First eight leads selected for the study and test datasets . . . . . 93

5.8 First twenty-three leads selected for the study dataset . . . . . . 94

5.9 Twelve lead system proposed for AF . . . . . . . . . . . . . 95

5.10 Errors for limited lead sets and standard ECG in the derivation of QRS complex, $\mathrm{P}$ wave and atrial fibrillatory waves . . . . . . . 96

5.11 Relative errors for limited lead sets and standard ECG in the derivation of QRS complex, $\mathrm{P}$ wave and atrial fibrillatory waves . 97

5.12 Errors in the derivation of atrial fibrillatory waves versus errors in reconstruction equal to a measured noise level . . . . . . . . . 98

6.1 Selection of AF segments free from ventricular activity . . . . . . 108

6.2 Examples of isopotential and wavefront propagation modes . . . 110

6.310 -second recordings showing the same AF segments as in Figures 6.4 and $6.5 \ldots \ldots \ldots$. . . . . . . . . . . . . . . . . . . . . . . . .

6.410 -second recordings showing the same AF segments as in Figures 6.4 and $6.5 \ldots \ldots \ldots . \ldots \ldots 112$ 
6.5 Wavefront propagation maps of the four selected AF segments from Figure 6.3 . . . . . . . . . . . . . . . . . . . . . . . . 114 



\section{Sumari}

La fibril-lació auricular (FA) és una de les arítmies cardiaques més comuns, afectant al voltant del $10 \%$ dels majors de 70 anys. A pesar de la seua alta incidència en la població, els mecanismes que desencadenen i mantenen la FA són incerts. Malgrat la existència de diversos tractaments quirúrgics i farmacològics, l'èxit dels tractaments contra la FA és molt baix. La causa d'aquesta baixa taxa d'èxit de les diferents teràpies és que no existeixen criteris de selecció de pacients que permeten pronosticar quina teràpia pot ser més efectiva per a cada pacient. Una de les formes que s'han proposat per a determinar el grau de gravetat de l'arítmia en cada pacient i, per tant, predir quin tractament és el més apropiat, és la mesura de l'organització auricular.

Aquesta tesi doctoral s'emmarca dins de la determinació no invasiva del grau d'organització espacial de l'activació del miocardi auricular a partir de l'estudi de registres multiderivació de l'electrocardiograma de superfície (ECG). L'ECG és una representació simplificada del camp elèctric del cor basada en les projeccions d'aquest camp elèctric en 8 eixos. Aquesta simplificació és considerada com acceptable en el cas de ritmes no fibril-lants en els quals l'activació miocàrdica pot ser modelada com un dipol. No obstant això, la seva validesa no ha estat demostrada per al cas de ritmes fibril-lants en els quals l'assumpció d'un model dipolar és qüestionable. Un dels objectius d'aquesta tesi ha estat l'avaluació de l'electrocardiograma de superfície per a l'obtenció de paràmetres espacials de les ones de FA. Es van comparar les representacions tridimensionals de les ones de FA registrades a partir de tres derivacions ortogonals amb les representacions tridimensionals estimades a partir del ECG, arribant a la conclusió que aquestes representacions estimades no són fidels a les representacions registrades. Els resultats del nostre estudi posen de manifest que la falta de dipolaritat de les ones de FA no permet l'estimació de paràmetres espacials a partir del ECG de superfície.

Per a tindre una representació fidedigna de les ones de FA és necessari, per tant, disposar d'un major nombre de derivacions electrocardiogràfiques que ens permeta conèixer els potencials en la superfície completa del tors durant FA. Un altre dels objectius d'aquesta tesi ha estat la determinació del nombre mínim de derivacions necessàries per a poder estimar amb exactitud els potencials en la superfície durant FA a partir de l'anàlisi dels estadístics del 
senyal. Aquest estudi ens ha permès determinar que el grau de complexitat de l'activació elèctrica durant FA obliga a l'ús del triple d'elèctrodes per a obtindre un grau d'exactitud similar en la representació de l'activació elèctrica al de l'electrocardiograma de superfície estandard per a ritmes no fibril-lants.

Fent ús del nombre suficient d'elèctrodes és possible representar de manera espacial la trajectòria de les ones de FA. Aquesta representació espacial ens ha permès observar diferents patrons d'activació elèctrica tals com la propagació d'un únic front d'ona o l'aparició de múltiples ones simultànies. Aquests patrons han pogut ser avaluats per primera vegada de forma no invasiva gràcies a aquest estudi i són coherents amb els patrons observats a partir de mesures invasivas. L'ús de representacions espacials de l'activació elèctrica sobre la superfície corporal durant FA pot permetre un major coneixement del grau d'organització auricular durant FA i pot ajudar, per tant, a seleccionar el tractament més apropiat per a cada pacient. 


\section{Sumario}

La fibrilación auricular (FA) es una de las arritmias cardiacas más comunes, afectando a alrededor del $10 \%$ de los mayores de 70 años. A pesar de su alta incidencia en la población, los mecanismos que desencadenan y mantienen la FA son inciertos. Aunque existen diversos tratamientos quirúrgicos y farmacológicos, el éxito de los tratamientos contra la FA es muy bajo. La causa de esta baja tasa de éxito de las diferentes terapias es que no existen criterios de selección de pacientes que permitan pronosticar qué terapia puede ser más efectiva para cada paciente. Una de las formas que se han propuesto para determinar el grado de gravedad de la arritmia en cada paciente y, por tanto, poder predecir qué tratamiento es el más apropiado es la medida de la organización auricular.

Esta tesis doctoral se enmarca dentro de la determinación no invasiva del grado de organización espacial de la activación del miocardio auricular a partir del estudio de registros multiderivación del electrocardiograma de superficie (ECG). El ECG es una representación simplificada del campo eléctrico del corazón basada en las proyecciones de este campo eléctrico en 8 ejes. Esta simplificación es considerada como aceptable en el caso de ritmos no fibrilantes en los que la activación miocárdica puede ser modelada como un dipolo. Sin embargo, su validez no ha sido demostrada para el caso de ritmos fibrilantes en los cuales la asunción de un modelo dipolar es cuestionable. Uno de los objetivos de esta tesis ha sido la evaluación del electrocardiograma de superficie para la obtención de parámetros espaciales de las ondas de FA. Se compararon las representaciones tridimensionales de las ondas de FA registradas a partir de tres derivaciones ortogonales con las representaciones tridimensionales estimadas a partir del ECG, llegando a la conclusión de que estas representaciones estimadas no son fieles a las representaciones registradas. Los resultados de nuestro estudio ponen de manifiesto que la falta de dipolaridad de las ondas de FA no permite la estimación de parámetros espaciales a partir del ECG de superficie.

Para tener una representación fidedigna de las ondas de FA es necesario, por tanto, disponer de un mayor número de derivaciones electrocardiográficas que nos permitan conocer los potenciales en la superficie completa del torso durante FA. Otro de los objetivos de esta tesis ha sido la determinación del 
número mínimo de derivaciones necesarias para poder estimar con exactitud los potenciales en la superfice durante FA a partir del análisis de los estadísticos de la señal. Este estudio nos ha permitido determinar que el grado de complejidad de la activación eléctrica durante fibrilación auricular obliga al uso del triple de electrodos para obtener un grado de exactitud similar en la representación de la activación eléctrica al del electrocardiograma de superficie para ritmos no fibrilantes.

Haciendo uso del número suficiente de electrodos es posible representar de manera espacial la trayectoria de las ondas de FA. Esta representación espacial nos ha permitido observar diferentes patrones de activación eléctrica tales como la propagación de un único frente de onda o la aparición de múltiples ondas simultáneas. Estos patrones han podido ser evaluados por primera vez de forma no invasiva gracias a este estudio y son coherentes con los patrones observados a partir de medidas invasivas. El uso de representaciones espaciales de la activación eléctrica sobre la superficie corporal durante fibrilación auricular puede permitir un mayor conocimiento del grado de organización auricular durante FA y puede ayudar, por tanto, a seleccionar el tratamiento más apropiado para cada paciente. 


\section{Abstract}

Atrial fibrillation (AF) is one of the most common cardiac arrhythmias, affecting around $10 \%$ of the population older than 70 years. In spite of its high incidence in the population, the mechanisms that trigger and maintain $\mathrm{AF}$ are uncertain. Although diverse surgical and pharmacological treatments exist, their success is very low. The cause of this low rate of success of the different therapies is the lack of a patient selection criterium that allows to predict which therapy will be more effective for each patient. Measurement of atrial organization has been proposed as a marker of the degree of severity of the arrhythmia in each patient, and thus a potential predictor of the success of therapeutical strategies.

This doctoral thesis is related to the noninvasive determination of the degree of spatial organization of the atrial activation from the study of multiple electrocardiographic recordings. The electrocardiogram (ECG) is a simplified representation of the electrical field of the heart based on its projection on 8 axis. This simplification is considered as acceptable in the case of non-fibrillating rhythms in which myocardial activation can be modelled as a dipole. However, its validity has not been demonstrated for the case of fibrillating rhythms in which the assumption of a dipolar model is questionable. One of the objectives of this thesis has been the evaluation of the suitability of the ECG for obtaining spatial characteristics of AF waves. Three-dimensional representations of AF waves obtained from three recorded orthogonal leads were compared to three-dimensional representations obtained from orthogonal leads estimated from the standard ECG. We concluded that estimated three-dimensional representations of AF loops are not accurate. The results of our study show that the lack of dipolarity of AF waves does not allow the estimation of spatial parameters from the standard ECG.

In order to have an accurate representation of AF waves it is necessary, therefore, to have a greater number of electrocardiographic derivations that allow us to determinate the potentials on the entire surface of the torso during AF. An objective of this thesis has been the determination of the minimum number of derivations that are necessary to accurately estimate surface potentials during AF by analyzing of the statistics of the signal. This study has allowed us to determine that the degree of complexity of the electrical activation during $\mathrm{AF}$ forces the use of three times more electrodes to achieve 
similar accuracy than the standard ECG for the representation of the electrical activation during non-fibrillanting rhythms.

Making use of the sufficient number of electrodes it is possible to represent the trajectory of AF waves in the space. This spatial representation has allowed us to observe different patterns of electrical activation such as the propagation of a single wavefront or the appearance of multiple simultaneous wavefronts. This is the first time these patterns, consistent with patterns obtained from invasive recordings, have been evaluated by making use of noninvasive recordings. Spatial representations of the electrical activation on the body surface during AF may provide a deeper knowledge of the degree of atrial organization during $\mathrm{AF}$ and can help, therefore, to select the most appropriate treatment for each patient. 


\section{Chapter 1}

\section{Introduction}

1.1 Motivation

\section{$1.2 \quad$ Objectives}

1.3 Structure of the thesis

Technological advantages in the field of electronics and computing are allowing the development of new instrumentation and methods in all scientific areas. Medicine is one of the areas that has greatly benefited from the introduction of new diagnostic and therapeutical techniques which has resulted in a major increase in the quality of life during the last years. Cardiology is, among other disciplines in medicine, one of the fields in which both the diagnosis and treatment have been greatly improved during the last decades. Introduction of the electrocardiogram (ECG) into the clinical practice has allowed the diagnosis of many cardiac diseases with an electrical manifestation and has been recently complemented by the use of imaging techniques such as echocardiography, computed axial tomography or magnetic resonance imaging, allowing the detection of hemodynamic disorders. Therapeutical procedures have also benefited from technical advantages: open chest surgery is being replaced by minimally invasive therapeutic techniques such as catheter-based ablation in some determined interventions, decreasing the risks associated with surgery.

Atrial fibrillation (AF), the most frequent cardiac arrhythmia, could not be described in animal experiments until 1874 when instrumentation for the measurement of the electrical activity of the heart was available [132]. The first recording of human AF was published in 1906 by W. Einthoven [30], but a significant background noise precluded the identification of atrial activity. It was not until 1910 when fibrillatory atrial waves could be recorded by Sir Thomas 
Lewis [82] who concluded that these waves could only be generated in the atria.

Since then, detection of AF has relied on the visual inspection of the ECG.

However, a minimum understanding of the mechanisms that trigger and maintain AF has not been possible until the recent development of multi-electrode arrays and optical mapping experiments. Current theories about the underlaying causes of AF suggest that the mechanism generating and/or perpetuating AF may be different in each patient. Determination of the clinical condition of each patient with current technology requires the attachment of multiple electrodes on the patient's heart. Electrodes can be attached to the outer surface of the heart (epicardium) during open chest surgery or to the inner surface of the heart (endocardium) during catheter ablation interventions and in either case the mechanism of AF may not be elucidated in all cases. Both epicardial and endocardial mapping have two main problems: (1) they offer a narrow perspective of the electrical activity of the atria which is restricted by the sampled area and (2) they are invasive, either highly invasive (epicardial mapping) or minimally invasive (endocardial mapping), which supposes a certain risk of death during the intervention, risk of infections and also elevated costs.

\subsection{Motivation}

The determination of physiological parameters that can guide the election of a therapy for patients with $\mathrm{AF}$ in a non-invasive manner would be of great interest. With this aim, several approaches have been proposed in the literature based on the measurement of the degree of electrical organization during AF. This organization degree can be studied either from a spatial or a temporal perspective. Temporal approaches usually analyze the spectral content of a single lead $[10,124]$ while spatial approaches analyze the variability in the directions of AF waves [102].

Previous studies regarding the spectral analysis of AF signals from the ECG have been restricted to the study of a single ECG lead at which AF waves appear with highest amplitude, being unable to quantify spatial variations. Spatial organization has been estimated by measuring spatial parameters from a vectorcardiogram (VCG) derived from the surface ECG [102]. However, the authors of this study did not determine whether a derivation method based on assumptions only valid for a non-fibrillating ventricle would be accurate for the derivation of the atrial VCG or not. From a theoretic point of view it is difficult to assume that the electrical activity during $\mathrm{AF}$ can be accurately represented by a single stationary dipole with its center located in the ventricles, but the degree of accuracy of this model has not been experimentally measured.

A more general model should not assume dipolarity of the electrical activity in the atria. Instead, it should include the possibility that certain areas on 
the body surface may reflect preferentially the activity of the closest region of the atria that may not be recorded at other surface areas. In order to record these local potentials a great number of electrodes should be needed as opposed to the limited number of electrodes in conventional ECG or VCG recordings. Previous studies have dealt with number and location of electrodes needed to record most meaningful information on the body surface by making use of body surface potential mapping (BSPM) systems in which the electrical activity in the whole body surface is recorded. In these studies the number of electrodes needed to account for most electrical information during ventricular depolarozation and repolarization were estimated to be of the order of 32 [85]. However, the number and location of electrodes needed to record most diagnostic information during $\mathrm{AF}$ has not been investigated in real BSPM recordings.

The use of non-standard lead systems or a BSPM system is only justified in case some diagnostic features can be extracted. Until now, no study in the literature has reported the generation of BSPM maps during AF. This may be caused by the low signal to noise ratio of AF recordings and the difficulty in interpreting and obtaining measurements from these chaotic signals. However, if these difficulties could be overcome and BSPM maps during AF could be constructed and measured, they could be potentially used for the assessment of the degree of organization in the atria and thus provide diagnostic information valuable for the selection of the best treatment in each patient.

\subsection{Objectives}

Extraction of diagnostic features in AF recordings apart from the simple detection of the arrhythmia in a non-invasive manner are of great interest for cardiologists nowadays. The aim of this dissertation is to evaluate the possibility of extracting spatial organization measurements from the surface ECG both from standard and non-standard recordings. The main objectives of this thesis can be established as:

- Evaluate the possibility of extracting spatial parameters from the standard ECG.

With this aim, a transform matrix for deriving orthogonal leads from the standard ECG and optimized for atrial electrical signals will be obtained. Spatial indicators will then be obtained both from recorded VCG signals and from derived VCGs by making use of this newly computed transform matrix and other transform matrices traditionally used. Spatial indicators of AF loops obtained from recorded orthogonal leads and derived orthogonal leads will be compared in order to determine the accuracy of derived spatial indicators and thus validate whether orthogonal leads can be derived from the standard ECG with accuracy. 
- Determine the number and location of exploring ECG electrodes most appropriate for the recording of atrial electrical activity during AF.

In order accomplish this objective, lead selection algorithms proposed in the literature will be evaluated on a large database for determining which lead selection algorithm offers a better performance. Then, we will apply this lead selection algorithm for the selection of lead sets optimized for the recording of $\mathrm{AF}$ signals and determine the minimum number of leads required to capture most electrical information present on the body surface as well as the location of these leads.

- Extract of spatial organization patterns from BSPM recordings during $\mathrm{AF}$.

New techniques for constructing BSPM maps will be developed given that there are no previous studies with this same objective. Spatial organization measurements similar to those reported in invasive studies will be computed from non-invasive BSPM recordings. These spatial organization measurements are of potential interest for determining the clinical condition of each patient and thus selecting the most appropriate treatment for each individual and plan clinical procedures in advance in a non-invasive manner.

\subsection{Structure of the thesis}

This thesis is structured in the following chapters:

Chapter 2: State of the art: This is a review chapter that reflects the state of the art in the different disciplines that are involved in this work. Non-invasive techniques for the recording of the electrical activity of the heart and the advantages and assumptions of each of them are detailed. Current knowledge of the mechanisms underlaying AF is summarized in this chapter. Technical issues such as derivation of orthogonal leads from the ECG and selection of leads from BSPM recordings are also discussed. Finally, methods employed in the literature for the measurement of atrial organization during AF are summarized.

Chapter 3: Databases: In this chapter the databases used for the development of this thesis are described. A public database of simultaneous VCG and ECG recordings was used. However, there are no existent databases containing BSPM recordings of patients with AF. For this reason we had to acquire new BSPM recordings during AF. In this chapter we describe the database that was acquired for the development of this thesis containing healthy subjects, patients with various diagnosis and patients with AF that are used for the development of our methods and for extracting our conclusions respectively. 
Chapter 4: Spatial characteristics from the ECG: In this chapter we evaluate spatial indicators obtained from the standard ECG. The suitability of existent derivation methods is evaluated and a derivation method optimized for atrial activity is described. The validity of derived VCGs to describe atrial loops during AF is evaluated by computing the accuracy of both existent derivation methods and this atrial-based derivation method.

Chapter 5: Optimization of ECG leads: Results of the evaluation of two different methods for the selection of leads in our large database of patients in sinus rhythm is reported. After evaluation in this large database, we report our results of the number and location of ECG leads that account for most electrical information during AF.

Chapter 6: Non-invasive mapping of human AF: In this chapter we describe the new methods that we have developed for the construction and evaluation of BSPM maps during atrial fibrillation. Our first observations on non-invasive human AF are reported.

Chapter 7: Discussion and conclusions: The contributions of this work are discussed in detail in this chapter, emphasizing the most relevant advances, but also their limitations. The fulfilment degree of the objectives established in the previous section is also analyzed in this chapter. Finally, some guides for future work are provided to overcome the limitations of this thesis and to extract the maximum profit from it.

Chapter 8: Contributions: The main scientific contributions derived from the work developed in this dissertation are listed in this section. The scientific framework in which this work has been involved is also described, including related research projects and international collaborations. 



\section{Chapter 2}

\section{State of the art}

\section{$2.1 \quad$ Introduction to electrocardiography}

\section{$2.2 \quad$ Atrial fibrillation}

\subsection{Spatial characterization of ECG signals}

\section{$2.4 \quad$ Optimization of ECG leads}

2.5 Organization degree of atrial fibrillation

In this chapter the main areas that are covered in this dissertation are explained, providing a sufficient background of the main concepts that are involved in the following chapters.

Fundamental concepts about heart electrophysiology and non-invasive techniques for the recording of this electrical activity of the heart are described. These techniques include the standard electrocardiogram, the vectorcardiogram and Body Surface Potential Mapping. Current knowledge of the pathophysiology of atrial fibrillation is also summarized in this chapter together with some unresolved issues which are tightly related with the content of this thesis.

The most relevant techniques that have been proposed in the literature for an improvement in the diagnosis of atrial fibrillation are described. These techniques include the analysis of spatial characteristics of AF signals from the surface ECG and from intracardiac recordings aiming at estimating the degree of organization in the atria during this arrhythmia. A different approach for improving the diagnosis of AF that is covered in this thesis is a repositioning of ECG electrodes. Methods for the determination of optimum lead sets are 
also described in this chapter.

\subsection{Introduction to Electrocardiography}

\subsubsection{The heart}

The heart is a muscular organ that lies in the center of the thoracic cavity whose primary function is to pump blood throughout the body by repeated, rhythmic contractions.

The heart is composed mostly of cardiac muscle called myocardium. It is divided by a central wall, or septum, into left and right halves. Each half consists of an atrium, which receives blood returning to the heart from the blood vessels, and a ventricle which pumps blood out into the blood vessels. The right side of the heart receives blood from the tissues and sends it to the lungs for oxygenation. The left side of the heart receives newly oxygenated blood from the lungs and pumps it to tissues throughout the body. Although blood flow in the left and right side of the heart is separated, the two sides of the heart contract in a coordinated fashion: first the atria contract together, then the ventricles contract. The heart has four valves: between the right atrium and ventricle lies the tricuspid valve, and between the left atrium and ventricle is the mitral valve. The pulmonary valve lies between the right ventricle and the pulmonary artery, while the aortic valve lies in the outflow tract of the left ventricle. Anatomy of the heart, valves and vessels is depicted in Figure 2.1.

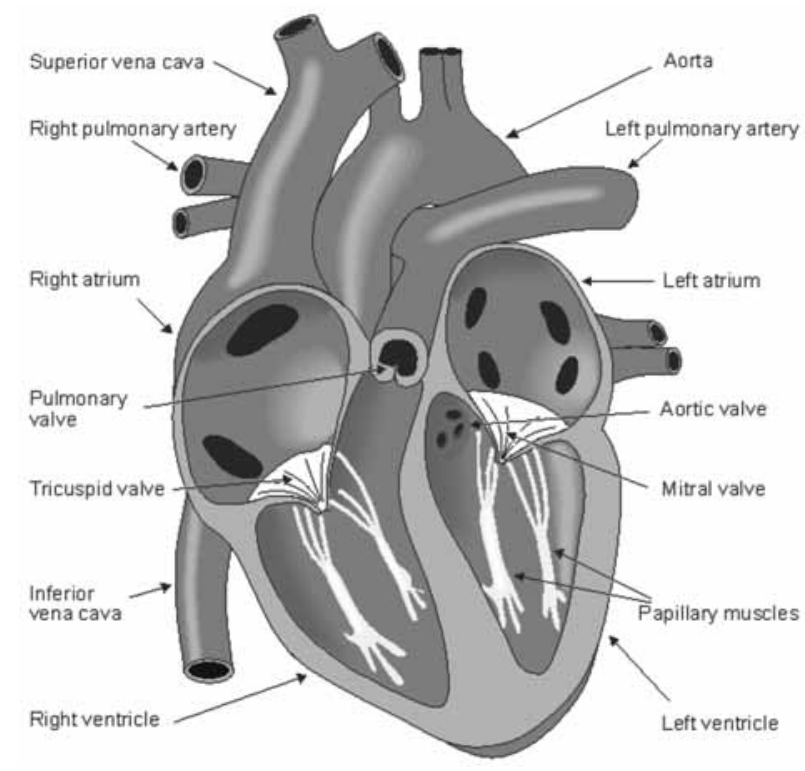

Figure 2.1: Anatomy of the heart, valves and vessels [90]. 
The rhythmic contractions of the heart depend on an electrical system which conducts electrical impulses along its conduction pathway. The marker of electrical stimulation, the action potential, is created by a sequence of ion fluxes through specific channels in the membrane of myocardial cells. If we were able to measure the intracellular potential with a voltmeter, the cathode measuring the potential inside the cell and the anode measuring outside, it could be observed that during a non-activity period the membrane voltage would remain constant around $-80 \mathrm{mV}$. In this state it is said that the cell is polarized, this is to say that the cell is at its resting potential. When a muscular cell is stimulated its membrane potential necessarily changes (there exists ion flow through the ionic channels of the membrane), characterized by a decrease in the normally negative resting voltage (depolarization) which triggers the activity of the cell, i.e. the contraction. After stimulation the membrane voltage returns to its original value (repolarization).

The impulse conducting system (Figure 2.2) consists of specialized cells that initiate the heart beat and electrically coordinate contractions of the heart chambers. The initiation of a cardiac cycle occurs in a small mass of specialized cells located in the wall of the right atrium with the ability to spontaneously depolarize and generate an action potential. This small mass of cells with pacemaker properties is called the sinoatrial (SA) node. Electrical impulses then spread rapidly through the internodal tracts that connect the SA node to the atrioventricular (AV) node, a group of autorhythmic cells near the floor of the right atrium. From the AV node, electrical impulses move into the bundle of His, which perforates the interventricular septum posteriorly. Within the septum, the bundle of His bifurcates into the left and right bundles. Finally, Purkinje fibers transmit the electrical impulses to the ventricular muscle. For a more detailed description of the anatomy and function of the heart see $[83,114]$.

\subsubsection{The surface electrocardiogram}

An electrocardiogram, or ECG, is a recording of the electrical activity of the heart made from electrodes placed on the surface of the skin. In 1908 Willem Einthoven published a description of the first clinically important ECG measuring system [31]. By using electrodes placed on the arms and legs, Einthoven described the tracings that can be observed during the cardiac cycle of a healthy subject and named the parts of the ECG as we know them today.

The ECG describes the electrical activity of the heart at each time instant of the cardiac cycle and represents a summation in time and space of the action potentials generated in myocardial cells. Each group of cells which is simultaneously depolarizing can be represented as an equivalent current dipole source to which a vector is associated, describing the dipole's time-varying position, orientation and magnitude. According to this dipolar model, the recorded ECG can be interpreted as the projection of the heart vector on the 


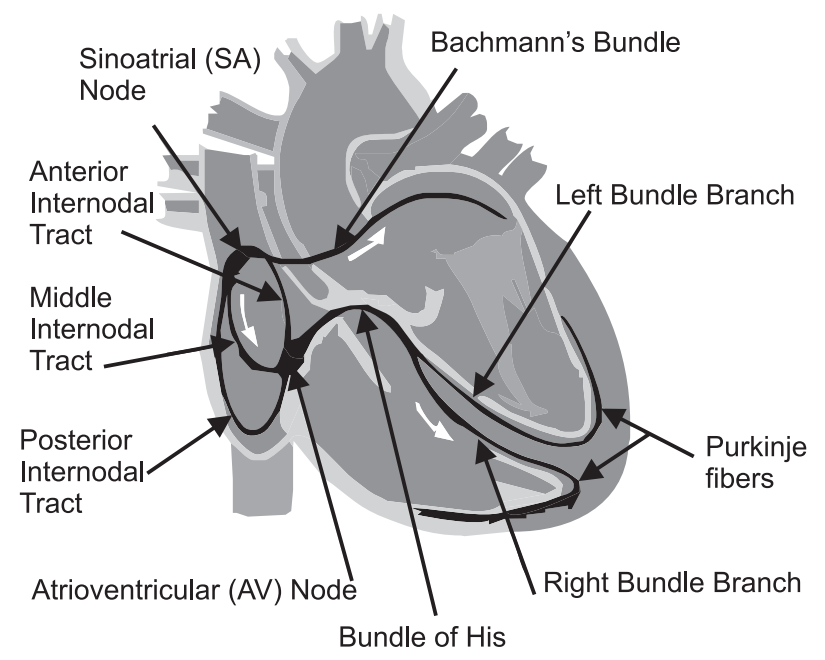

Figure 2.2: Intrinsic conduction system of the heart

lead vector defined by the exploring electrodes [90] as it is depicted in Figure 2.3 .

An standard ECG consists on the recording of six bipolar limb leads and six precordial leads which are constructed from nine exploring electrodes whose position on the body surface can be observed in Figure 2.4.

The Einthoven limb leads (standard leads) are defined in the following way:

$$
\begin{aligned}
& \text { Lead I: } \quad V_{I}=\phi_{L}-\phi_{R} \\
& \text { Lead II: } \quad V_{I I}=\phi_{F}-\phi_{R} \\
& \text { Lead III: } \quad V_{I I I}=\phi_{F}-\phi_{L}
\end{aligned}
$$

where

$$
\begin{aligned}
V_{I} & =\text { the voltaje of Lead I } \\
V_{I I} & =\text { the voltaje of Lead II } \\
V_{I I I} & =\text { the voltaje of Lead III } \\
\phi_{L} & =\text { potential at the left arm } \\
\phi_{R} & =\text { potential at the right arm } \\
\phi_{F} & =\text { potential at the left foot }
\end{aligned}
$$

Electrode positions on the left arm, right arm and left leg define an equilateral triangle with the heart at its center called Einthoven's triangle.

Three additional limb leads were obtained by Goldberger by measuring the potential between a single limb electrode and the midpotential of the two remaining electrodes, chosen as reference. These additional leads are called 

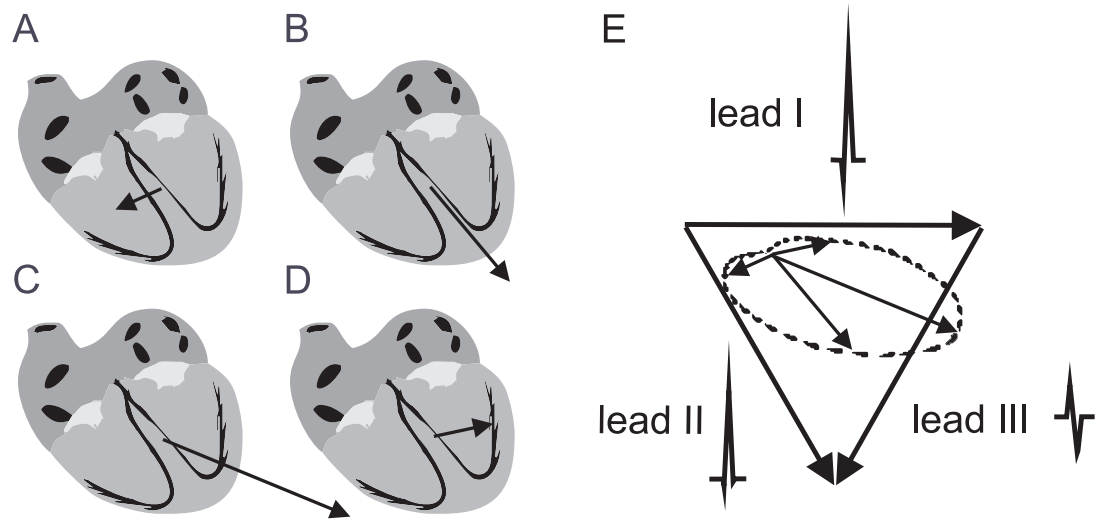

Figure 2.3: The electric heart vector and its projection on limb leads. Panels A-D show the equivalent heart vectors at 4 instants during ventricular depolarization. Panel E shows the projection of heart vectors on limb leads, with different amplitudes and morphologies of the signals.

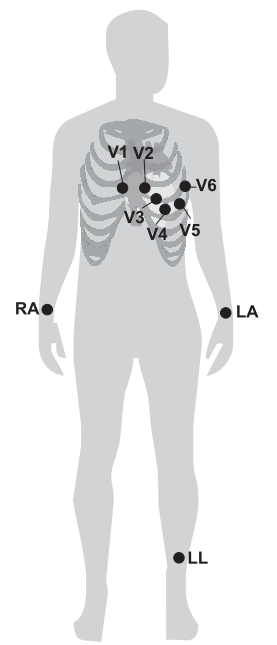

Figure 2.4: Standard positions of the ECG electrodes 
augmented leads due to the augmentation of the signal. Notice that the Goldberger augmented leads are fully redundant with respect to the Einthoven leads I, II and III, with the following relationship:

$$
\begin{aligned}
& a V_{R}=\phi_{R}-\frac{\phi_{L}+\phi_{F}}{2} \\
& a V_{L}=\phi_{L}-\frac{\phi_{R}+\phi_{F}}{2} \\
& a V_{F}=\phi_{F}-\frac{\phi_{R}+\phi_{L}}{2}
\end{aligned}
$$

The six limb leads examine the electrical forces in the frontal plane of the body. However, since electrical activity travels in three dimensions, recordings from a perpendicular plane are also essential. This is accomplished by the use of six electrodes placed on the anterior and left lateral aspect of the chest, creating the precordial leads, by convention labelled $V_{1}, \ldots V_{6}$. These leads, $V_{1}-V_{6}$ are located over the left chest as described in Figure 2.4. The points $V_{1}$ and $V_{2}$ are located at the fourth intercostal space on the right and left side of the sternum; $V_{4}$ is located in the fifth intercostal space at the midclavicular line; $V_{3}$ is located between the points $V_{2}$ and $V_{4} ; V_{5}$ is at the same horizontal level as $V_{4}$ but on the anterior axillary line; $V_{6}$ is at the same horizontal level as $V_{4}$ but at the midaxillary line. Precordial leads are related to a central terminal (called Wilson Central terminal or WCT) which is defined as the center of Einthoven's triangle and can be computed as average of the voltages measured on the right and left arms and the left leg:

$$
V_{W C T}=\frac{\phi_{L}+\phi_{R}+\phi_{F}}{3}
$$

The normal electrocardiogram is illustrated in Figure 2.5. Depolarization of the atria result in a first deflection in the electrocardiogram named $\mathrm{P}$ wave. After the atria depolarizes, the electrical impulse is delayed in the AV node and an inactive period can be observed in the ECG. Then, depolarization of the ventricles occur, and it is reflected in the QRS complex. Notice that the amplitude of the QRS complex is higher than the $\mathrm{P}$ wave because more myocardial mass is involved in ventricular depolarization than in atrial depolarization. Some time after depolarization of the ventricles, there is a last wave in the ECG reflecting repolarization of the ventricles named $\mathrm{T}$ wave. Different intervals can be defined in the ECG according to the names of the waves already defined. The PQ segment is the time between the beginning of the $\mathrm{P}$ wave and the beginning of the QRS complex and is an indirect measurement of the atrioventricular delay. The QT (or QRST-T) segment comprises the time between the onset of the QRS complex and the offset of the $\mathrm{T}$ wave and represents a measurement of the time at which ventricular 
cells are depolarized (approximate measurement of the action potential duration). The time between the offset of the QRS complex and the onset of the $\mathrm{T}$ wave is called ST segment. The ST-T segment is defined between the offset of the QRS complex and the offset of the $\mathrm{T}$ wave and represents the repolarization time of the ventricles. The TP segment comprises the time between the offset of the $\mathrm{T}$ wave and the onset of the $\mathrm{P}$ wave. TP segment represents a resting time in the heart at which no electrical activity is taking place in a healthy heart. The time interval between the offset of the $\mathrm{T}$ wave and the onset of the QRS complex is called TQ segment and represents the resting period of the ventricles. The time between two adjacent QRS complexes is called RR interval.

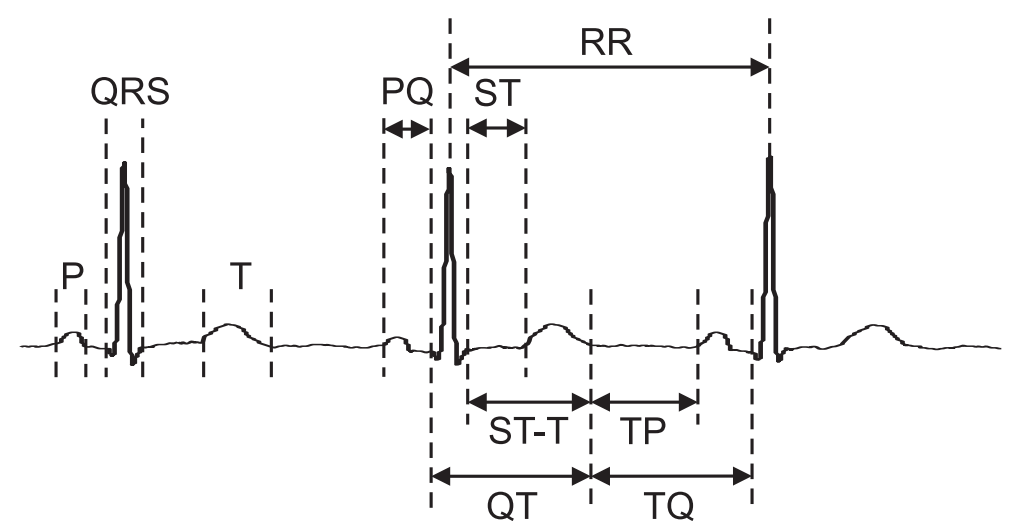

Figure 2.5: Normal ECG tracing. Waves and intervals defined for the ECG are also displayed.

\subsubsection{The vectorcardiogram}

Because it is possible to describe the electrical activity of the heart as an equivalent dipole or heart vector with reasonable accuracy, one representation form of this electrical activity of the heart is in vector form. This spatial representation of the electrical activity of the heart is called vectorcardiogram (VCG). An ideal lead system for vectorcardiography consists of three orthogonal leads and with the same sensitivity [20]. Although many lead systems for vectorcardiography were proposed in the first half of the $20^{\text {th }}$ century, the only lead system that is used in clinical practice is that proposed by Ernest Frank in 1956 [44] based on his published data of image surface [42]. Frank used models of the human torso filled with tap water in which a small finite dipole was inserted. He first determined where the dipole should be located inside the human model and for that reason he determined the electrical center of the ventricular depolarization in the human heart [41]. 
Frank did not expect that this center would be the WCT since Wilson assumed that the body is spherical in shape with the heart centrally located inside the torso. He performed cancellation experiments both in a human subject and in a torso model of this same subject and searching for the same mirrowing patterns of the QRS complex in the model and in the human subject. After performing these experiments, he defined where the center of the electrical depolarization of the ventricles should be placed. As Frank himself stated in [41], his determination of the center of the heart was only valid for the QRS complex as the centers of $\mathrm{P}$ and $\mathrm{T}$ activity are not the same as that of the QRS complex.

In Frank's VCG lead system, seven electrodes are used in order to obtain three weighted orthogonal components (see Figure 2.6). The rational of using seven electrodes instead of the four electrodes that are strictly necessary is to decrease the error due to interindividual variation in the heart location and body shape. In this system, electrodes A, C, E, I and M are situated on the same transverse level (the fifth intercostal space). Electrode $\mathrm{A}$ is placed on the left midaxillary line; electrode I is placed on the right midaxillary line, electrodes $\mathrm{E}$ and $\mathrm{M}$ are applied on the midline anteriorly and posteriorly, respectively, and finally, electrode $\mathrm{C}$ is located midway between the anterior midline (electrode $\mathrm{E}$ ) and the left midaxillary line (electrode A). Electrode $\mathrm{F}$ is placed on the left leg, and electrode $\mathrm{H}$ on the back of the neck. $\mathrm{X}, \mathrm{Y}$ and $\mathrm{Z}$ components are derived according to the following relationships:

$$
\begin{aligned}
& V_{X}=0.610 \cdot V_{A}+0.171 \cdot V_{C}-0.781 \cdot V_{I} \\
& V_{Y}=0.655 \cdot V_{F}+0.345 \cdot V_{M}-1.000 \cdot V_{H} \\
& V_{Z}=0.736 \cdot V_{M}+0.133 \cdot V_{A}-0.264 \cdot V_{I}-0.374 \cdot V_{E}-0.231 \cdot V_{C}
\end{aligned}
$$

The lead axes of Frank's lead system are labelled $x$ for transverse, $y$ for vertical and $z$ for sagittal. Two of these lead axes are used to display VCG planes, so that the transverse plane uses the $x z$ axes, the sagittal plane uses $z y$ axes and the frontal plane uses the $x y$ axes. A normal vectorcardiogram can be observed in Figure 2.7.

The VCG contains both electrical and spatial information of the events taking place in the heart. This spatial information can be useful to determine the location at which a specific electrical event takes place or the contribution of a specific tissue region on the morphology of VCG loops. An important spatial parameter that can be used as a risk stratifier in post myocardial patients is the angle between the spatial QRS loop and the T wave loop [4], which is based in the concept of the ventricular gradient and is therefore an expression of the spatial inhomogeneities in local duration of excitation. The VCG may also be applied to the study of atrial loops. Date et al. [22] could identify the role of the cardiac tissue surrounding the pulmonary veins in the formation of the $\mathrm{P}$ wave and thus the changes that pulmonary vein isolation 


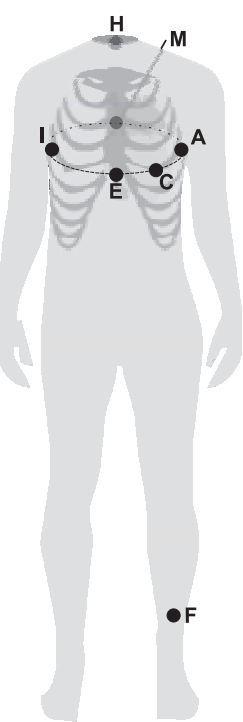

Figure 2.6: Electrode positions of Frank's vectorcardiographic system.

TRANSVERSE PLANE
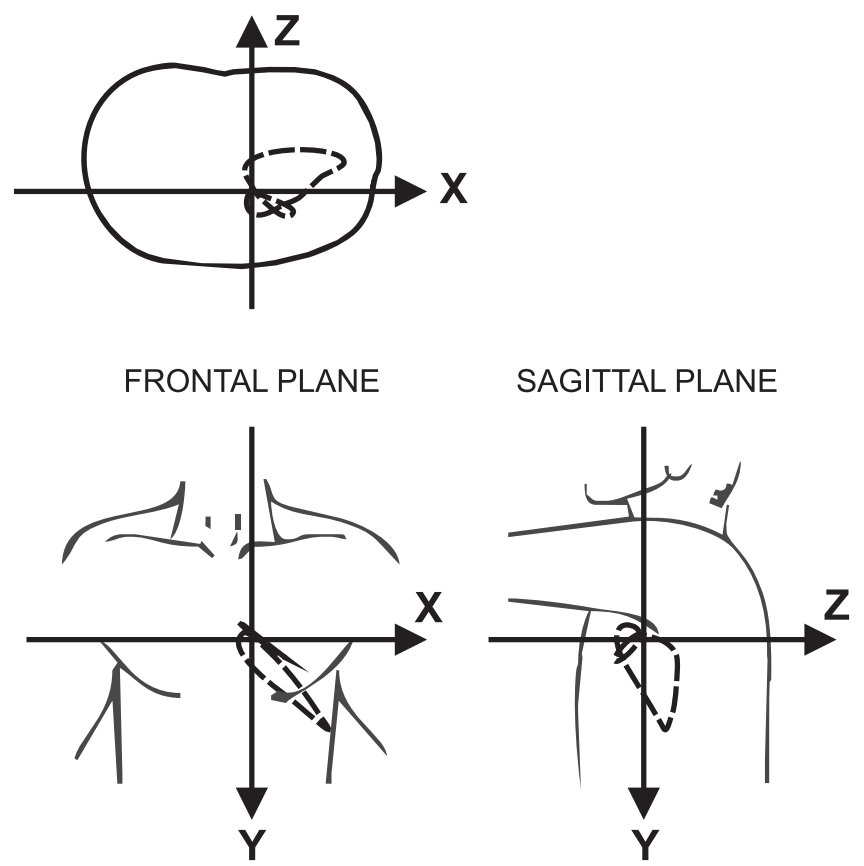

Figure 2.7: A normal vectorcardiogram in the transverse, frontal and sagittal planes 
produces on the $\mathrm{P}$ loop.

\subsubsection{Body Surface Potential Mapping}

Body Surface Potential Mapping (BSPM) was, historically, the first method used in human electrocardiography [133]. Unlike the standard 12-lead ECG, which records electrical signals from nine sites, BSPM depicts the cardiac electrical field as reflected on the entire surface of the torso.

Later studies suggested that the cardiac electrical field could be approximated by a single rotating dipole [14] and thus the use of a BSPM system was unnecessary. Supporting this hypothesis, Frank estimated that approximately 95 per cent of the QRS complex at any body surface point in a healthy subject could be attributed to a fixed-location equivalent dipole [43]. However, this single dipole theory was contradictory with the observations by Taccardi [126] who reported that during larger portions of the ventricular depolarization than those studied by Frank, several maxima and minima could be observed on the body surface (see Figure 2.8). Taccardi attributed this finding to the existence, within the heart, of multiple, separate groups of electrically active fibers each group exerting a preponderant influence on certain regions of the chest surface, what he called proximity potentials. After the studies from Taccardi, Horan et al., by performing principal factor analysis found that the first three factors accounted for an average of 85.4 per cent of the available information on the man chest [64]. During the last decades, many studies have demonstrated that BSPM contain more diagnostic and prognostic information than the elicited from the 12-lead ECG [127]. Despite these advantages, BSPM has not supplanted electrocardiography nor has it become a routinely used clinical method.

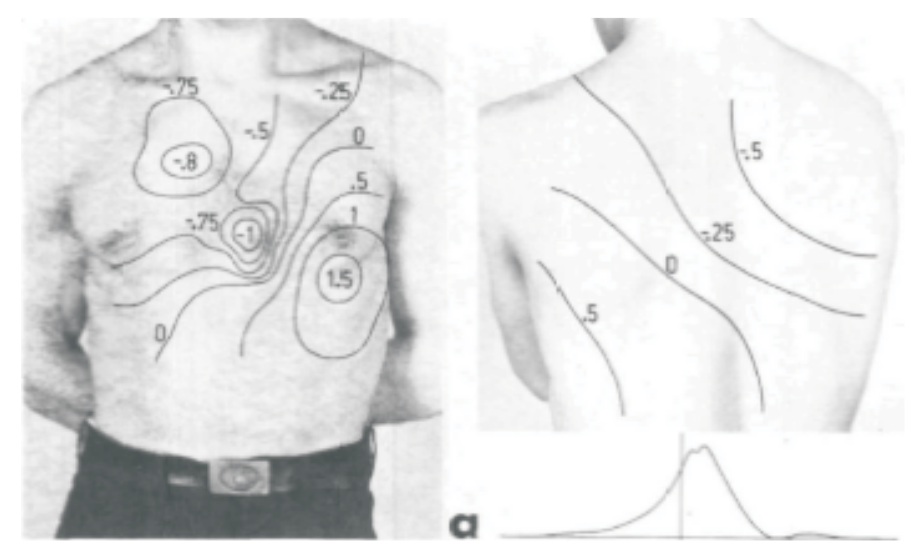

Figure 2.8: Example of isopotential maps of the thoracic surface of a healthy subject showing the existence of two simultaneous potential minima [126] 
Number of electrodes used in BSPM range from 32 up to 240. There is no agreement in their number neither in their location, and every research group uses its own configuration. Electrodes are always placed on the anterior and the posterior chest from the level of the top of the sternum to low below the rig margins. More electrodes are commonly placed on the anterior than on the posterior torso because higher potentials and steeper potential gradients are found on the anterior than on the posterior chest wall. Most authors defend that there is great redundancy in BSPM recordings and thus the number of electrodes with independent information ranges from 30 to 64 [88, 61].

First BSPM recordings were obtained by photographing the screen of cathode ray tubes while displaying up to five simultaneous ECG tracings $[43,126]$. In current BSPM systems, potentials from each electrode are simultaneously amplified, converted to digital form at a sampling rate of 250-4096 samples per second and stored at computers where maps are automatically computed by using specialized software.

The most common format for displaying body surface electrical information is the equipotential map in which the electrical potential at any position on the body surface is represented in a given time instant according to a color scale. Also, isointegral maps of QRS, ST-T or QRST waveforms have been widely used as they summarize in a single map the information contained in tens of instantaneous maps. Among integral maps, the QRST integral map has the property of expressing the body surface distribution of the ventricular gradient [135].

The application that has accounted for most BSPM studies is the diagnosis of myocardial infarction. Flowers et al. described mid and late abnormalities in BSPM maps of QRS complexes of patients with a recent acute myocardial infarction (AMI) not detectable in the standard ECG [39] and in patients not showing abnormalities in the QRS complex $[99,96]$. Location and size of myocardial infarction has been determined from isointegral analysis of BSPM maps [129] as well as analysis of maps of abnormal Q waves and changes in ST elevation [6]. These findings have allowed an improvement in diagnosis of anterior and inferior AMI with discriminant analysis [73, 74] and later other infarction locations [97] even when associated with additional complications such as bundle branch block (BBB) [95, 94]. Diagnosis of AMI is improved by the use of BSPM with an overall sensitivity of 80 per cent compared to 57 per cent with the standard ECG [103].

Body Surface Mapping has been specially useful in the understanding Brugada Syndrome which lead to a change in the position of the ECG electrodes for diagnosing this disease, placing electrodes on the parasternal second and third intercostal spaces [112, 111]. Also, BSPM was shown to be useful in differentiating patients with symptomatic Brugada syndrome from those with asymptomatic Brugada Syndrome [60].

Other studies have allowed the identification of at least seven preexcitation 
sites in patients with Wolf-Parkinson-White syndrome [5], localization of ectopic focus of atrial tachycardia $[118,117]$, rotation direction in atrial flutter $[115]$ and localization of atrial fibrillation trigger sites [116].

\subsection{Atrial fibrillation}

\subsubsection{Pathophysiology}

Atrial fibrillation (AF) is a supraventricular arrhythmia in which the electrical activation in the atria shows an uncoordinated pattern. This chaotic electrical activity results in a loss of effective contraction of the atria which is reflected on the surface ECG by a lost of the $\mathrm{P}$ wave that appears during sinus rhythm and the presence of AF waves (see Figure 2.9).

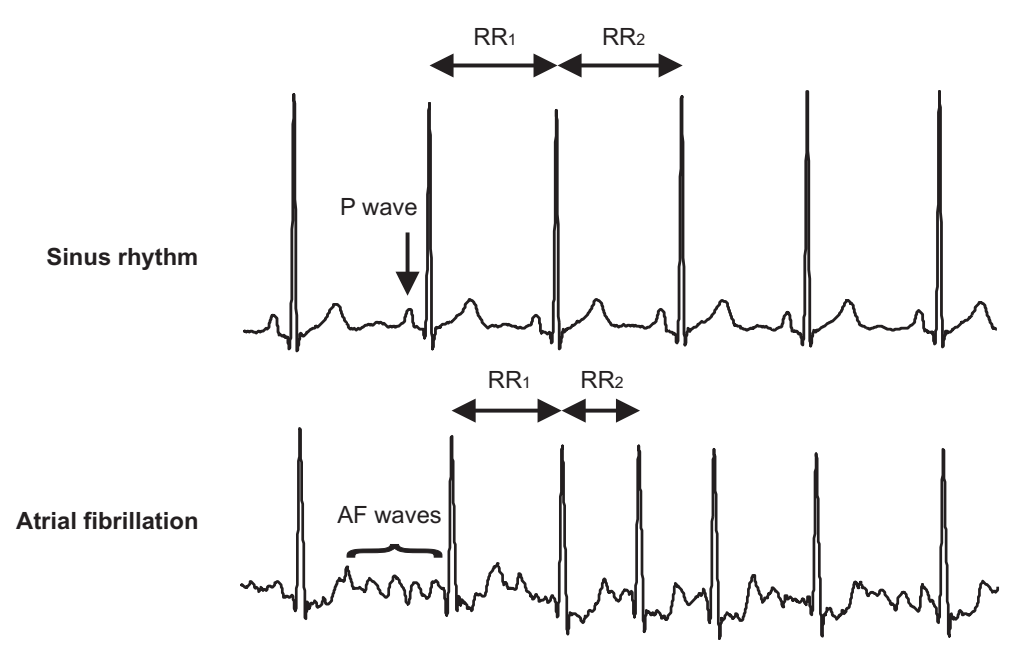

Figure 2.9: ECG tracings during sinus rhythm and atrial fibrillation

Normally, heart rate is controlled by the SA node and adapts to body's needs in a range of 60 beats per minute at rest to 200 beats per minute at peak exercise. During AF, atrial cells fire at rates of 300 to 600 beats per minute. If each atrial impulse were conducted to the ventricles, such a rapid ventricular rate would result in a loss of the effective contraction of the ventricles and a rapid death. This is prevented by the filtering function of the AV node allowing only a part of the impulses to reach the ventricles. As a consequence of the irregular electrical activity of the atria and the effect of the AV node, the ventricular rate is also irregular (see Figure 2.9).

The mechanisms underlaying development and maintenance of AF are still not well understood. During the twentieth century different theories about the origin of AF coexisted: existence of a rapidly discharging atrial ectopic foci (Figure 2.10a), by a single reentry circuit (Figure 2.10b) or by multiple 
functional reentrant circuits (Figure 2.10c). For both the rapid focal and single reentrant circuit theories, irregularity is presumed to result from interactions between wavefronts produced by the main generator while for the multiple circuit reentry irregularity is a consequence of the primary arrhythmia mechanism [100].
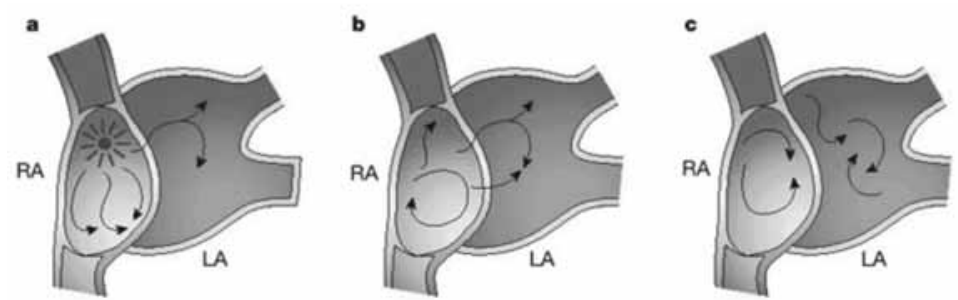

Figure 2.10: Conceptual models of atrial fibrillation. (a) ectopic foci, (b) single reentrant circuits and (c) multiple reentrant circuits [100]

Recent observations on the initiation of AF from the area of the pulmonary veins [59] and optical mapping studies that found mother rotors and fibrillatory conduction as the mechanism underlying perpetuation of AF [69] suggest that a complex mechanism in which ectopic activity, single circuit reentry and wavefront fractionation may all be involved.

A better understanding of a number of processes collectively referred to as atrial remodeling has added to the complexity of the pathophysiology of AF [1]. Remodeling is the name given to the alteration of several properties in the atria as a consequence of AF that favours the ease of inducing and mantaining the arrhythmia [100]. Remodeling can be subdivided into electric remodeling, contractile remodeling and structural remodeling. Electrical remodeling refers to the alteration of electrophysiological properties in the atria that result in the shortening of atrial refractoriness. It develops within the first days of $\mathrm{AF}$ and contributes to an increase in stability of AF. Contractile remodelling refers to a loss of contractility in myocardial muscle that persists after the restoration of sinus rhythm. Atrial structural remodeling is associated to atrial fibrosis which occurs when $\mathrm{AF}$ is perpetuated during long periods of time. One of the main reasons to make distinction among types of remodeling is that the first two types of remodeling seem to be reversible, whereas the last is less so [137].

Clinicians distinguish three basic types of atrial fibrillation: paroxysmal, persistent and permanent [45]. Paroxysmal AF is characterized by $\mathrm{AF}$ episodes that initiate and terminate spontaneously. In this type of $\mathrm{AF}$ triggered activity appears to be the dominant factor and thus elimination of the trigger is expected to terminate the arrythmia. Persistent AF requires an intervention for termination to occur. In this type of $\mathrm{AF}$, electrical remodeling is present while none or little structural remodeling exists and thus this form of AF is reversible. Permanent AF is characterized by extensive fibrosis in the 
atria that makes impossible to restore sinus rhythm despite aggressive therapy.

\subsubsection{Epidemiology}

Atrial fibrillation is the most common sustained arrhythmia, affecting an estimated 2.3 million adults in the United States [134]. The prevalence of atrial fibrillation increases markedly with age in older adults, from $<1$ per cent in adults younger than 55 to 10 per cent in adults older than 80 years. Projections are that by the year 2025, 3.3 million adults in the United States will have atrial fibrillation, with $>35$ per cent of affected persons 80 years or older.

In most patients, $\mathrm{AF}$ is associated with some other cardiac or non-cardiac process including hypertension, congestive heart failure, diabetes and previous myocardial infarction. However, some patients seem to have AF as their only diagnosis. In those patients genetic factors may be important [13], although genetic linkage has only been reported in a small part of the population with $\mathrm{AF}$.

Hospitalizations for atrial fibrillation as the principle diagnosis increased from 154.086 in 1985 to 376.487 in 1999 in the United States. In the same period, hospitalizations for atrial fibrillation as a secondary diagnosis increased from 787.750 to 2.283 .673 . In-hospital mortality among patients with a principle diagnosis of atrial fibrillation was $<1$ per cent in patients 35 to 74 years of age, 1.5 per cent in those 75 to 84 years of age and 3 per cent in patients 85 years or older [134].

\subsubsection{Treatment of atrial fibrillation}

One of the primary clinical problems associated with AF is thromboembolism. The loss of atrial contraction that leads to stasis of blood in the atria is the responsible to clot formation and the occurrence of thromboembolism which tends to propagate to other organs including the brain, kidneys or the heart itself [100]. Thromboembolic risk is reduced by the administration of anticoagulants, but they increase the risk of bleeding complications.

In contrast to other cardiac arrhythmias for which safe and effective therapies have been developed, AF continues to be a challenge for both pharmacologic and non-pharmacologic approaches to treatment. Pharmacologic approaches include antiarrhythmic drugs while non-pharmacologic approaches include termination of the arrhythmia by applying controlled electrical impulses (electrical cardioversion) and the destruction of the cardiac tissue that triggers atrial fibrillation (ablation therapy).

The best treatment will be dependent on the basic mechanism of perpetuation of $\mathrm{AF}$ in each individual. Patients whose underlaying $\mathrm{AF}$ mechanism is trigger activity may benefit from isolation of the proarrythmic 
tissue that is most commonly found in the proximity of the pulmonary veins. In patients with persistent AF in which electrical remodeling has initiated to take place, the arrythmia can terminate after electrical cardioversion. Addition of antiarrhythmic drugs to electrical cardioversion have shown to decrease recurrence rates [23]. For patients with permanent AF in which structural remodeling is the dominant feature, attempts to restore sinus rhythm are abandoned and their preferable therapy aims at controlling the heart rhythm by a pharmacologic approach. Measurement of the organization degree of the electrical activity of the atria during AF may help in determining the mechanism maintaining AF in each individual.

\subsection{Spatial characterization of ECG signals}

\subsubsection{Derivation of orthogonal leads from the standard electrocardiogram}

The 12-lead ECG is the most extended noninvasive technique in the diagnosis of AF. However, the standard ECG does not provide spatial information that can be obtained with a vectorcardiogram (VCG). However, the VCG is not usually recorded during clinical practice and it can be derived from the 12-lead ECG. All the transformation methods described in the literature have modelled the derivation of orthogonal leads from the standard electrocardiogram as a linear transformation, assuming the simplification of the voltage at any point in the surface as a projection of a fixed vector.

$$
\left[\begin{array}{lll}
\mathbf{x} & \mathbf{y} & \mathbf{z}
\end{array}\right]^{T}=\mathbf{L}\left[\begin{array}{llllllll}
V_{1} & V_{2} & V_{3} & V_{4} & V_{5} & V_{6} & I & I I
\end{array}\right]^{T}
$$

where $\mathbf{L}$ is a $3 \times 8$ matrix that defines the linear transformation that allows the derivation of $\mathbf{x}, \mathbf{y}, \mathbf{z}$ leads from the eight independent leads of the standard ECG.

The most common transformation matrix used for the derivation of orthogonal leads from the standard ECG is the so-called Dower's inverse transform. Dower published in 1968 a lead synthesizer of standard ECG leads from Frank's orthogonal leads [25]. Dower based his calculation of all the coefficients of his transformation matrix on the same torso model that Frank used for the definition of his VCG system [42]. It must be emphasized that Frank used a torso model filled with water with a fixed electrical dipole located in a similar position to the position of the ventricles inside the thoracic surface. Dower visually estimated the level at which electrodes corresponding to V1 to V6 should be placed on Frank's image surface of a human torso and derived his coefficients directly using Frank's experiments, although after a few trials he decided to consider the chest leads to be slightly higher than his original estimate because the morphology of $\mathrm{P}$ waves and $\mathrm{T}$ waves was not accurately reproduced [28]. 
As previously stated, Dower defined his transformation matrix for the derivation of standard leads from recorded orthogonal leads, aiming at reducing the electronics needed for data acquisition. After testing his derivation method he concluded that although the differences between the ECG and the derived ECG would not always appear negligible to an engineer, to a cardiologist, who perceives such differences in the light of his experience with intra- and inter-individual variation and their interpretative significance, the differences appear minor $[27,26]$.

Later studies have made use of Dower's results for the opposite derivation: obtention of orthogonal leads from the standard 12-lead ECG. For this derivation they used the inverse of the coefficient matrix published by Dower, the so-called Dower's inverse transform:

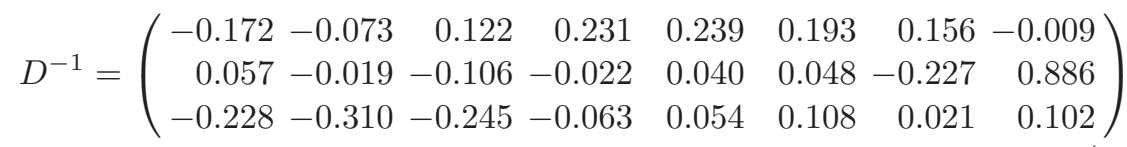

A number of studies have tested the accuracy of Dower's inverse transform and other transforms for the derivation of orthogonal waves from the standard ECG with disparate results. Levkov proposed a transformation matrix based on least squares optimization and concluded that his transformation matrix offered a better reconstruction of orthogonal leads than the lead vector approach proposed by Dower [81]. Kors et al. [77] compared a transformation matrix computed by multiple regression (see equation 2.11) to Dower's inverse transform and concluded that his proposed transform was preferable to other methods tested.

$$
K=\left(\begin{array}{rrrrrrrr}
0.38 & -0.07 & -0.13 & 0.05 & -0.01 & 0.14 & 0.06 & 0.54 \\
-0.07 & 0.93 & 0.06 & -0.02 & -0.05 & 0.06 & -0.17 & 0.13 \\
0.11 & -0.23 & -0.43 & -0.06 & -0.14 & -0.20 & -0.11 & 0.31
\end{array}\right)
$$

Edenbrandt et al. [29] concluded that Dower's inverse transform achieved good results in derivation of orthogonal leads, although they did not compare to the transformation matrices previously defined by Levkov or Kors. Finally, Rubel [110] et al. compared the transformation matrices proposed by Dower, Levkov and Kors and concluded that Kors' matrix offered better reconstruction performance over all the matrices tested. Despite of these results, Dower's inverse transform is the only transformation matrix that is used in practice and even some ECG recorders implement it for the derivation of orthogonal leads from the standard ECG.

Aforementioned studies focused on the evaluation of the performance of different transform matrices for the derivation of QRS complexes and/or the T wave. Carlson et al. [16] tested the accuracy of Dower's inverse transform for 
the derivation of $\mathrm{P}$ waves in patients with a history of AF. They correlated Frank leads with derived leads in a lead-by-lead basis and concluded that Dower's inverse transform preserved P wave morphology.

\subsubsection{Study of spatial characteristics of derived atrial loops during atrial fibrillation}

Derived atrial loops were first used for the characterization of atrial flutter [101]. Planes of best fit to each atrial loop were calculated. Flutter loops were found to be consistent with expected anatomic orientation of the reentry circuit parallel to the tricuspid valve. In a later study, $\mathrm{Ng}$ et al. [102, 105] applied a similar method for the characterization of AF waves. They found that some patients presented $30 \%$ or more vector loops with planes within 30 degrees of each other (see Figure 2.11). They associated this lower variability in the direction of AF loop planes to a higher degree of organization. They also stated that they could determine the direction of the dominant patterns of activation. Based on these observations, they claimed that the study of AF loops derived from the standard ECG allowed them to evaluate the degree of organization of AF signals.

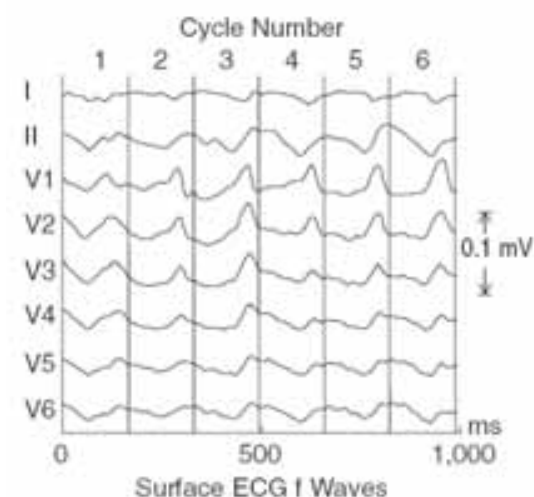

(a)
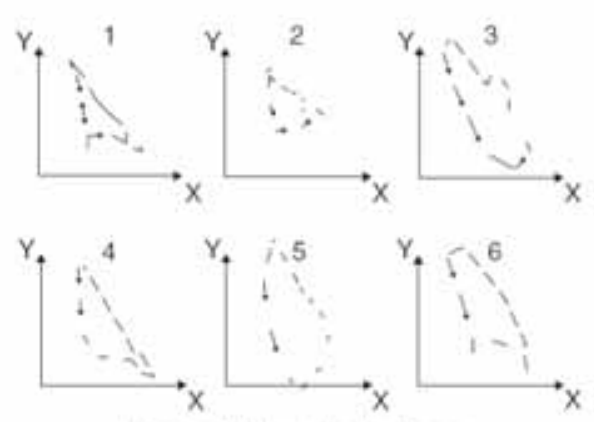

Derived $\mathrm{f}$ Wave Vector Loops

(b)

Figure 2.11: Vector loops of six consecutive AF waves [105]

Following the observations from Ng, Richter et al. [106] studied the spatial characteristics of AF from the surface ECG. They derived orthogonal leads from the standard ECG by using Dower's inverse transform. They correlated the planes of best fit of AF loops and other spatial parameters such as planarity of the loops with atrial fibrillatory frequency, and they observed a weak correlation among spatial parameters and AF frequency. They concluded that those spatial parameters are useful for the characterization of the degree of organization of the atria in a non-invasive manner.

Van Oosterom et al. [131], however, questioned whether the actual atrial 
electrical dipole could be accurately represented by Frank's VCG system which was defined for representing the dipole vector related to ventricular activity. They used a biophysical model of AF in which the atrial dipole was computed from Gabor-Nelson equations and potentials on the entire body surface were derived by using different torso models. They observed that a shift in Frank's electrode positions one intercostal space up together with weighting coefficients calculated specifically for atrial activity decreased the error in estimation of atrial electrical dipoles from $39 \%$ to $28 \%$. A retrieval of the atrial electrical dipole from the standard electrocardiogram different from that proposed by Dower and optimized for AF signals produced 30\% error.

\subsection{Optimization of electrocardiographic leads}

The ECG allows the detection of many pathological heart conditions: arrythmias, bundle branch block, abnormal wave intervals duration, etc. However, many heart diseases affect to a localized segment of the myocardium (i.e. occlusion of a coronary artery) and consequently, electrical manifestations of these localized cardiac diseases are also localized on the body surface [87]. The standard ECG does not sample all regions of the chest and often undersamples electrical manifestations that reflect an underlying disease. Alternative lead systems are being proposed during the last years in journal special issues and scientific symposiums which aim at capturing most diagnostic ECG information.

\subsubsection{Determination of the number and location of optimum electrocardiographic leads}

BSPM is the most complete technique for the characterization of the electrical activity of the heart in a non-invasive manner. The tedious of the procedure of attaching tens of electrodes to each patient and the added cost of the recording equipment have relegated BSPM to research studies. However, BSPM studies have revealed that the standard electrocardiogram does not always contain diagnostic features that can be observed with BSPM maps. BSPM information has been used to define lead systems that contain most diagnostic information but a limited number of leads, and thus more practical than a complete BSPM system. A lead system with a limited number of leads is considered to be optimal if it consists of the minimum number of electrodes required for achieving an accurate reconstruction of body surface potentials [61].

Various approaches can be followed to estimate how many leads are necessary to obtain most relevant information of cardiac electrical activity [61]:

- Analysis of the spatial correlation among recording sites [88]. 
- Analysis of the number of independent signals based on the minimum description length estimate [130].

- Identification of the added diagnostic value of a selection of BSPM leads $[73]$.

\section{Spatial correlation approach}

Lux et al. [88] proposed a sequential algorithm for the selection of limited lead sets from BSPM recordings. This lead selection method is an iterative process where, at each iteration, the lead that maximizes the information contained in the lead selection is added to the lead set from the previous iteration.

Considering the potential $L \times K$ matrix $\mathbf{X}$, where $L$ is the number of electrodes, $K$ is the number of samples and each row represents the sequence of potentials at each electrode, its covariance matrix $\mathbf{C}_{\mathbf{x}}$ is computed as:

$$
\mathbf{C}_{x}=\mathrm{E}\left[\mathbf{X X}^{\mathrm{T}}\right]
$$

where $\mathrm{E}[\cdot]$ is the expectation operator.

Each step of the iterative algorithm involves the estimation of the non-selected leads from the leads selected in the previous step. The estimation of the $N$ non-selected leads $\hat{\mathbf{X}}_{\left\{i_{1}, \ldots, i_{N}\right\}}$ from the $M$ selected leads is performed following a least squares optimization approach:

$$
\hat{\mathbf{X}}_{\left\{i_{1}, \ldots, i_{N}\right\}}=\mathbf{T X}_{\left\{j_{1}, \ldots, j_{M}\right\}},
$$

where $\mathbf{X}_{\{\cdot\}}$ is a matrix containing the rows of $\mathbf{X}$ corresponding to the indexes in $\{\cdot\},\left\{i_{1}, \ldots, i_{N}\right\}$ are the indexes of the non-selected leads, $\left\{j_{1}, \ldots, j_{M}\right\}$ the indexes of the selected leads and $\mathbf{T}$ is the transform that optimizes the estimation, which can be computed as follows:

$$
\mathbf{T}=\mathbf{C}_{12}^{T} \mathbf{C}_{11}^{-1}
$$

where $\mathbf{C}_{12}$ is the $M \times N$ cross covariance matrix of $\mathbf{X}_{\left\{j_{1}, \ldots, j_{M}\right\}}$ and $\mathbf{X}_{\left\{i_{1}, \ldots, i_{N}\right\}}$ :

$$
\mathbf{C}_{12}=\mathrm{E}\left[\mathbf{X}_{\left\{\mathrm{j}_{1}, \ldots, \mathrm{j}_{\mathrm{M}}\right\}} \mathbf{X}_{\left\{\mathrm{i}_{1}, \ldots, \mathrm{i}_{\mathrm{N}}\right\}}^{\mathrm{T}}\right]
$$

and $\mathbf{C}_{11}$ is the $M \times M$ covariance matrix of $\mathbf{X}_{\left\{j_{1}, \ldots, j_{M}\right\}}$ :

$$
\mathbf{C}_{11}=\mathrm{E}\left[\mathbf{X}_{\left\{\mathrm{j}_{1}, \ldots, \mathrm{j}_{\mathrm{M}}\right\}} \mathbf{X}_{\left\{\mathrm{j}_{1}, \ldots, \mathrm{j}_{\mathrm{M}}\right\}}^{\mathrm{T}}\right]
$$

Let us now define the error matrix $\mathbf{X}_{e}$ as the difference between the true potentials $\mathbf{X}_{\left\{i_{1}, \ldots, i_{N}\right\}}$ and their estimates $\hat{\mathbf{X}}_{\left\{i_{1}, \ldots, i_{N}\right\}}$

$$
\mathbf{X}_{e}=\mathbf{X}_{\left\{i_{1}, \ldots, i_{N}\right\}}-\hat{\mathbf{X}}_{\left\{i_{1}, \ldots, i_{N}\right\}}
$$


as well as its covariance matrix $\mathbf{C}_{e}$ :

$$
\mathbf{C}_{e}=\mathrm{E}\left[\mathbf{X}_{\mathrm{e}} \mathbf{X}_{\mathrm{e}}^{\mathrm{T}}\right] \text {. }
$$

From the covariance matrix $\mathbf{C}_{e}$, an index $I$ that accounts for the global amount of new information (i.e. non redundant information with respect to the information contained by the selected leads) provided by each of the non selected leads is defined:

$$
I(n)=\frac{\mathbf{c}_{n} \mathbf{c}_{n}^{\mathrm{T}}}{c_{n n}}
$$

where $\mathbf{c}_{n}$ is the vector corresponding to the coefficients in the $n^{\text {th }}$-row of $\mathbf{C}_{e}$, and $c_{n n}$ is the diagonal coefficient in row and column $n$.

The row that maximizes the index $I$ is associated to the electrode with highest covariance values with all the other sites. The lead selection algorithm is an iterative procedure where (1) the potentials at the non-selected sites, $\hat{\mathbf{X}}_{\left\{i_{1}, \ldots, i_{N}\right\}}$, are estimated from the leads selected in the previous iteration (2) the error matrix $\mathbf{X}_{e}$ and its covariance matrix are computed $\mathbf{C}_{e}(3)$ the index $I$ is computed for each electrode and (4) the electrode $i_{n}$ that maximizes $I$ is selected. This algorithm is initialized with $\hat{\mathbf{X}}_{\left\{i_{1}, \ldots, i_{N}\right\}}$ being a zero $L \times K$ matrix and hence $\mathbf{X}_{e}$ being initially equal to $\mathbf{X}$, and is repeated until the desired number of electrodes is reached.

By using this approach, Lux et al. concluded that 32 appropriately located leads provides an accurate estimate of the total body surface distribution of electrocardiographic potentials [85]. However, for a given number of leads there are many lead sets which will perform comparably with insignificant differences between them. In this sense, Lux pointed that there is no absolute optimum array, but a many near optimal sets.

Later studies have compared the performance of this lead selection method and semi-exhaustive search algorithms. Finlay et al. [37] implemented a sequential algorithm in which at each iteration the lead that allows a better reconstruction of the potentials on the entire body surface is added to the leads included in the previous iteration. At each iteration, all leads not included in the previous iteration are added to the previous lead set, then potentials on the entire body surface are estimated from all included leads and the error in reconstruction is measured. Although the position of selected leads differs from one method to another, they reported a similar level of performance.

\section{Signal independency approach}

Uijen et al. proposed in later studies $[130,61]$ the application of information theory for the determination of the number of independent signals in BSPM recordings. They formulated their detection of the number of independent signals from the minimum description length (MDL) function. 
By using this information criteria, the number of independent signals in BSPM signals in the QRS complex and the $\mathrm{T}$ wave was found to be of the order of 10 [61] for all individuals.

This MDL approach allows the determination of the number of independent signals in BSPM recordings but fails at defining the number of ECG leads needed to retrieve those independent signals as well as the location of those leads. The number of electrodes needed to obtain these independent signals needs to be larger than the number of independent signals. The first reason is that it is impossible to select set of leads which all measure independent information of the ECG. Also, the eigenvectors associated with the independent signals are different for different subjects and for subjects with different diseases.

Later studies [67] have developed an iterative search algorithm for the selection of optimum lead sets based on an information content criterion related to this MDL approach. This algorithm iteratively adds to the lead set from the previous step the lead that maximizes a parameter which aims to reflect the amount of information contained in a certain lead set. The parameter $\alpha_{M}$ is derived from the singular value decomposition (SVD) of the $M$ selected leads $\mathbf{X}_{\left\{j_{1}, \ldots, j_{M}\right\}}$, according to the following expression:

$$
\alpha_{M}=\frac{\sigma_{M}}{\sigma_{1}},
$$

where $\sigma_{M}$ and $\sigma_{1}$ are the smallest and largest singular values, respectively. According to [67], the larger $\alpha_{M}$, the more information is contained in the lead set, and consequently the higher performance is achieved. This lead selection algorithm is an iterative procedure where (1) the lead $i_{n}$ of the non-selected group is added to the $M$ selected leads from the previous step, hence obtaining the potential matrices $\mathbf{X}_{\left\{j_{1}, \ldots, j_{M}, i_{n}\right\}}$, for each $n, 1 \leq n \leq N$ (2) update $M=$ $M+1$ and compute $\alpha_{M}$ for each of those matrices. This provides an index $\alpha_{M}$ for each of the non-selected leads (3) the electrode $i_{n}$ that maximizes $\alpha_{M}$ is selected and added to the lead set output in the previous iteration. This algorithm is repeated until the desired number of electrodes is reached.

\section{Discriminant analysis approach}

Kornreich et al. proposed to select optimum lead sets from BSPM recordings by determining the leads that showed the greatest statistically significant signal difference for separating data from diagnostic group pairs [73, 76, 74].

Discriminant maps can be computed by subtracting at each electrode site the mean voltage of one group of patients from the other group of patients. These discriminant maps were mostly used to discriminate between healthy subjects and myocardial infarction patients $[73,74]$ or ventricular hypertrophy patients [76]. Discriminant indexes for each electrode site and each group comparison were obtained by further dividing each resulting difference by the composite standard deviation computed from the population under 
consideration. Multivariate analysis was used for optimal separation of patients based on discriminant indexes together with other diagnostic measurements namely duration of the ST-T segment, mid-QRS voltage measurement, etc. This analysis allowed the identification of optimum leads for discriminating patients with myocardial infarction, most of them outside the area sampled by the conventional ECG and showing better classification rates than the standard ECG [75].

However, implementation of such an approach is problematic in that one must know the diseases to be tested before selecting the leads to be used for optimal detection and discrimination [87].

\subsubsection{Repositioning of electrocardiographic leads for the study of atrial fibrilation}

Most studies regarding the optimization of electrocardiographic leads have focused in the optimization of the amount of information recorded during ventricular activity. However, the origin of electrical activity during atrial contraction differs from the electrical activity in its spatial location, orientation and amplitude. This different location and impulse propagation pattern makes the optimization of electrocardiographic leads for the study of atrial activity a different problem to that of ventricular activity.

The existence of indiscernible $\mathrm{P}$ waves in the standard electrocardiogram in some patients has stimulated the search for non-standard leads that better capture atrial activity. The so-called Lewis lead recorded between the second and forth intercostal spaces, adjacent to the sternum, has been occasionally used for recording atrial activity. However, Madias et al. [89] found no difference in amplitude in P waves recorded with the standard ECG and the Lewis lead.

Lux et al. followed their method proposed for the optimization of electrocardiographic leads as explained in section 2.4.1 for the optimization of recording leads for the $\mathrm{P}$ wave by exploring the entire body surface during atrial depolarization. They found that P waves $33 \%$ taller than in lead II could be obtained in non-standard lead placements [86].

Electrical activity during AF shows a completely different pattern of activation which is nearly chaotic as opposed to a more regular activation of the ventricle during non-fibrillating rhythms. This makes optimization of electrocardiographic leads for the study of AF a separate problem than optimization of electrocardiographic leads for the study of the $\mathrm{P}$ wave.

Husser et al. proposed a non-standard set of surface electrodes for the analysis of atrial fibrillation [66] by empirically placing more electrodes anteriorly and posteriorly over the atria. They found that different characteristics of AF waves could be identified in the anterior and posterior torso and thus more information about the fibrillatory process in each patient could be obtained by placing electrodes on non-standard positions. 
A more systematic approach for determining the position of non-standard leads for the study of AF was followed by Ihara et al. [67]. They followed an information content optimization criterion as explained in section 2.4.1 for a repositioning of the 12-lead electrocardiographic leads. Body surface potentials of simulated AF were used to determine both the number of independent leads and the optimum location of a limited lead set for the study of AF. The MDL function revealed the presence of almost 60 independent signals. However, a selection of 64 leads was shown to contain 16 independent signals, which agreed with the conclusions previously obtained for non-fibrillating systems in the sense that a number of electrodes higher than the number of independent signals are needed in order to capture all independent information. An alternative 12lead system to the standard electrocardiogram was proposed for a retrieval of a higher number of singular values than the standard ECG (see Figure 2.12).
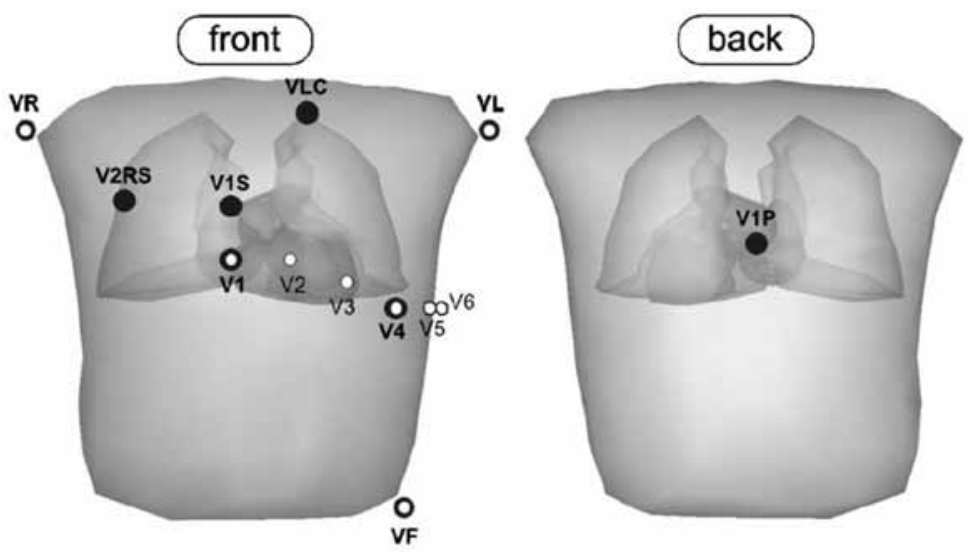

Figure 2.12: Adaptation of the standard 12-lead electrocardiogram based on an information content index applied to simulated AF signals [67]. White dots indicate the standard 12-lead positions. Heavy dots indicate electrode locations of the proposed lead system.

The accuracy of this lead system in representing the atrial electrical dipole was tested by van Oosterom et al. [131] and compared to the standard ECG, Frank's lead system, a modified version of Frank's system and a set of 64 electrodes in simulated AF signals. While the standard ECG produced errors of $30 \%$ and a modified version of Frank's VCG system produced 27\% error, the lead system proposed by Ihara produced $22 \%$ error. Errors lower than $10 \%$ could only be achieved by employing 64 leads. 


\subsection{Organization degree of atrial fibrillation}

Although AF was often described as a disorganized or random phenomenon, there is evidence supporting an underlaying organization in some patients. Gerstenfeld et al. [46] showed transient similarities in the direction of wavelet propagation in the majority of their patients under study. These transient similarities are known as transient linking, or a maintenance in the direction of atrial depolarization waves within a range of 30 degrees. This underlaying organization can be studied both in the temporal and spatial domains.

\subsubsection{Quantification of the temporal organization of AF}

Organization of electrical activity during AF can also be quantified in a temporal domain at any recording electrode. An organized electrical activation results in some degree of periodicity in EGM recordings. This periodicity can be determined by analyzing the similarity in morphology of consecutive atrial beats in lead by lead in endocardial recordings [36].

Most authors have estimated temporal organization of AF electrograms by analysis in the frequency domain [35]. The magnitude spectrum of the AF wavefront often shows a single dominant peak with other multiple peaks in the range of $2-20 \mathrm{~Hz}$. Organization index is defined as the area under the dominant peak and three of its harmonics. This organization index is theorized to represent the number of propagating AF wavelets: single wavelets with a dominant wavefront will manifest in a high organization index, while multiple wavelets manifest in many frequency components and low organization indexes.

Frequency analysis of endocardial electrograms have two main drawbacks: (1) electrograms are an invasive measurement with associated costs and risk for the patient and (2) they offer a narrow field of view of the electrical activity of the atria. For this reason, many research groups have focused in determining the atrial organization degree from the surface ECG given that spectral features of surface ECG recordings during AF have shown to be highly correlated with intracardiac measurements [108].

Analysis of the frequency content of AF signals from the surface ECG is complicated by the simultaneous presence of atrial and ventricular activity. Characterization of spectral features of AF signals require for the isolation of atrial activity from ventricular activity in the surface ECG. Atrial and ventricular activity overlap in the spectrum and thus they cannot be separated by linear filtering. Several techniques have been proposed in the literature for the extraction of AF signals from the surface ECG. A review of this techniques can be found in [11]. Average beat subtraction is the most commonly used technique for AF signal extraction and relies on the fact that $\mathrm{AF}$ is uncoupled to the ventricular activity [120]. It is based on the regularity of ventricular components compared to the irregularity in atrial components. A QRS-T template can be obtained by averaging successive QRST waves which is 
subtracted to the ECG signal at every position at which a QRS-T complex is detected. Since average beat subtraction is performed in individual leads, it becomes sensitive to alterations in electrical axis, which are manifest in large QRS-T residuals. This effect can be suppressed by using spatiotemporal QRS-T cancellation in which the average beats of adjacent leads are combined with the average beat of the analyzed lead in order to produce optimal cancellation [123]. For performing a lead-by-lead cancellation, principal component analysis (PCA) can be used to extract both the main QRS-T pattern and its dynamic changes over time [18]. Another approach to atrial signal extraction exploits the property that atrial and ventricular activities arise from different bioelectrical sources. These two sources can be separated by independent component analysis thanks to the differences in their statistical properties [107]. A comparison of the performance of these atrial signal extraction algorithms can be found in [79].

Having the atrial signal extracted from the surface ECG, its spectrum can be obtained using either Fourier-based spectral analysis of time-frequency analysis. Fourier-based spectral analysis is usually approached by using non-parametric analysis (e.g. Welch's periodogram) in order to reduce the variance in the spectral estimation. Segment length is critical in fourier-based analysis since it determines spectral resolution, and thus the use of at least a few seconds is recommended. The power spectrum of AF signals extracted from the ECG is concentrated in the 4-9 Hz band as it can be observed in Figure 2.13. However, since there are reasons to believe that the fibrillatory waveforms have timedependent properties, time-frequency analysis can be used in order to obtain a more detailed temporal characterization of atrial signals [125].
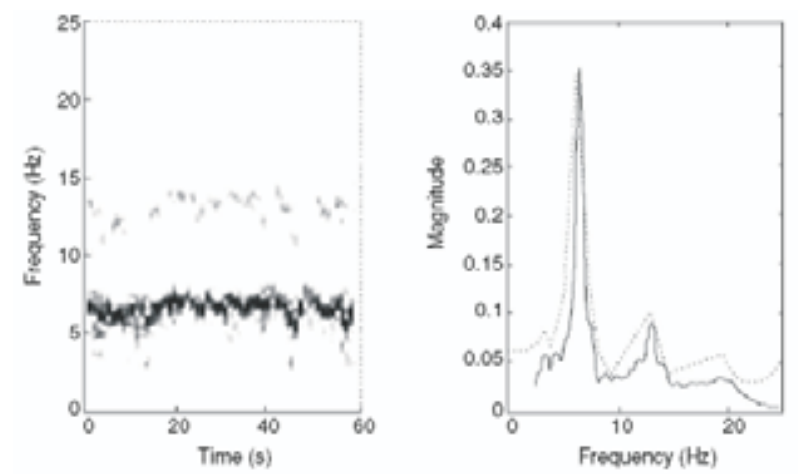

Figure 2.13: Time-frequency analysis of AF signals from surface ECG lead V1 [125] 


\subsubsection{Quantification of the spatial organization of AF}

Mapping of the human right atrium during induced AF confirmed the existence of a certain degree of spatial organization of AF. Konings et al. [71] used a 244-lead epicardial mapping electrode for recording right atrial activation during induced AF in 25 patients. Although in all cases the right atrium was activated by non-uniform wavefronts, there were large interindividual variations in the organization of the wavefronts. In some patients, the right atrium was activated by a single wavefront with minimum degree of blockade. In other patients, activation of the right atrium was highly fragmented by various arcs of conduction block. Konings et al. proposed a criteria for quantifying the degree of complexity of atrial activation during AF based on the analysis of epicardial isochronal maps (see Figure 2.14):

- Type I: single broad wavefronts propagating without significant conduction delay, exhibiting only short arcs of conduction block or small areas of slow conduction not disturbing the main course of propagation.

- Type II: activation patterns characterized either by single waves associated with a considerable amount of conduction block and/or slow conduction or the presence of two wavelets.

- Type III: presence of three or more wavelets associated with areas of slow conduction and multiple arcs of conduction block.
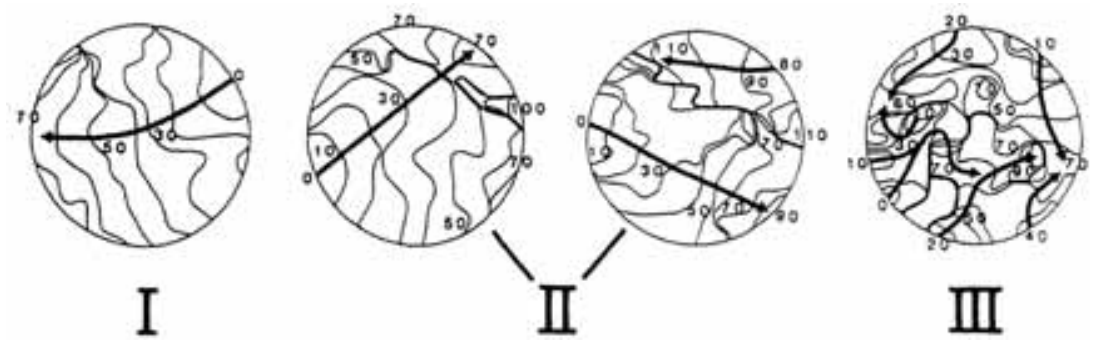

Figure 2.14: Mapping criteria for classification of the electrical organization degree in AF from [71]

Patients were classified according to their predominant type of fibrillation. An incidence of more than $50 \%$ of type I or type III beats was chosen as a boundary between the types of fibrillation. In this way, $40 \%$ patients were classified as having type I, $32 \%$ patients as having type II and $28 \%$ patients as having type III AF. This activation pattern in the right atrium was consistent in the left atrium in 5 of the 8 patients in which the left atrium was simultaneously mapped, whereas in 3 patients the degree of organization in the right atrium was different from that in the left atrium. According to 
Konings et al., activation patterns found could be related to the mechanism maintaining AF in each patient: type III fibrillation is based on multiple reentering wavelets while type I fibrillation is more consistent with a single macroreentrant circuit.

In further studies from the group of Allesie et al. [65] epicardial maps of induced AF were automatically analyzed and the direction of each wavefront was extracted. Electrical organization during AF was then estimated from the measurement of the anisotropy in conduction, given by the ratio of the velocity of conduction of longitudinal and transverse propagation.

The main drawback of epicardial mapping is the need for open chest surgery. A less invasive approach involves the recording of endocardial electrograms (EGMs). Organization indexes can be extracted by comparing endocardial EGMs of two or more sites, based on the observation that atrial beats during organized electrical activity resemble to other atrial beats, while disorganized atrial activity results in atrial beats that do not resemble beats recorded at different sites. Many approaches have been reported in the literature for measuring this resemblance among atrial beats in ECG recordings. Ropella et al. [109] proposed the use of the coherence spectrum, a quantification of the relation between spectral components that shows increasing values with increasing organization indexes. Botteron et al. [12] measured the degree of correlation among activation sequences during $\mathrm{AF}$ in five closely spaced electrodes. Sih et al. [113] proposed the quantification of AF organization by mean squared error in the linear prediction between two consecutive cardiac EGMs. More recently, Faes et al. [36] described a morphology-based approach in which regularity of atrial beats depends on a shape analysis.

The amount of spatial information conveyed in these organization indexes extracted from endocardial recordings is limited by the number of EGMs that can be acquired simultaneously. Although there are no technical complications for simultaneously recording a high number of electrodes, there are practical complications related to the attachment of the electrodes to the atrial wall without compromising blood circulation through the atria. Also, these techniques do not allow to plan the procedure in advance to the intervention. Promising results have arisen from the use of a non-contact multielectrode catheter showing similar activation patterns to those described by Konings [70]. However, clinical evidence supporting the reliability of this technique is needed before its implantation in clinical practice.

\subsubsection{Understanding the mechanisms of atrial fibrillation}

Recent experimental studies with animal models have improved the current understanding of the mechanisms of atrial fibrillation and its relation to spectral analysis. Skanes et al. [119] identified sequential wavefronts with temporal periodicity and similar propagation patterns by optical mapping 
studies in Langendorff-perfused sheep hearts. In those recordings in which spatiotemporal periodicity was found, the cycle length of spatiotemporal periodic waves correlated well with the dominant frequency in bipolar EGMs, although this correlation was higher in the left atrium than in the right atrium. Sources of periodic activity were seen occasionally as rotors (see Figure 2.15.A) or periodic breakthroughs although in most cases (see Figure 2.15.B) periodic waves entered the mapping area from the edge of the field of view.

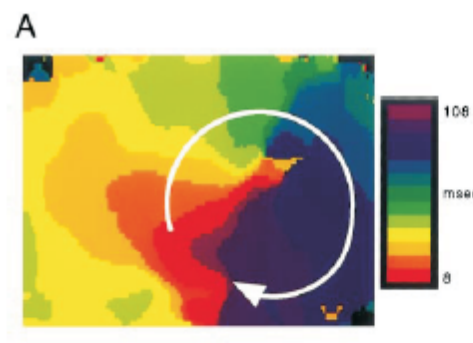

B Pseudo EG of LA

c
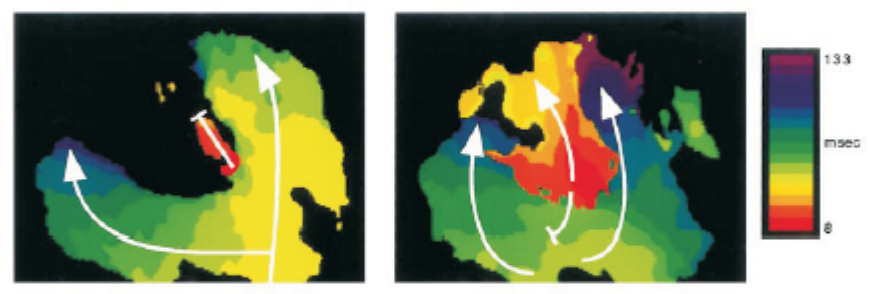

D

Pseudo $E G$ of RA

E

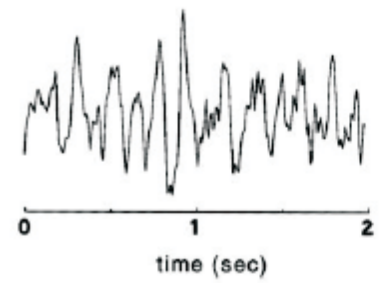

Bipolar EG

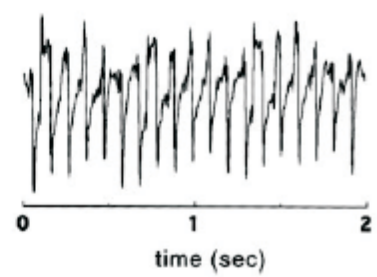

Figure 2.15: Isochrone maps and pseudo-EGs constructed from LA and RA optical recordings of an episode of AF. [119]

These rotors have been interpreted as the primary engines of fibrillation [69] as opposed to the multiple wavelet theory postulated by Moe [98]. Rotors may be stationary or they may drift. In either case, they may give rise to vortices of electrical waves (spiral waves) or complex fractionation patterns typical in AF. Observation of multiple wavefronts in previous mapping experiments may result from the fractionation of a single source. 
The location of periodic sources responsible for the maintenance of AF has found to be the left atrium in most cases according to optical mapping experiments by the group of Jalife et al. in isolated sheep hearts. By mapping the dominant frequencies (DF) in both atria, different DF domains could be observed [93] together with a left-to-right impulse propagation. Electrograms at the boundaries of areas with different DFs present a higher degree of fractionation (and higher frequency components) than at which the dominant frequency is constant. These observed left-to-right frequency gradients and fractionated electrograms were explained by high-frequency sources in the left atrium that are intermittently blocked in the right atrium, giving rise to lower activation frequencies and complex patterns in the right atrium [68]. Results in humans are also consistent with this left-to-right frequency gradients in paroxysmal AF, while no frequency gradient was found in persistent AF [80]. This hypothesis could explain the findings by Holm et al., who found a spatial dispersion in cycle length in patients with chronic AF by using precordial and esophageal recordings, suggesting intraindividual frequency distribution [63].

\subsubsection{Clinical implications}

Recent clinical and experimental studies have allowed the development of therapeutic strategies for terminating AF, including pulmonary vein ablation and electrical or pharmacologic cardioversion.

The hypothesis of rapid wavefronts emanating from a relatively stable rotor near the pulmonary veins supported by a left-to-right frequency gradient are consistent with the termination of the arrhythmia preferentially in patients with paroxysmal AF. Determination of whether the arrhythmia has its origin in the pulmonary veins may be of interest for predicting the success of this therapy in each individual patient.

Determination of the degree of atrial organization may be also of interest for selecting a cardioversion procedure. Everett et al. demonstrated that atrial defibrillation was more effective when electrical shocks were delivered during periods of high organization [32].

Also, atrial organization previous to electrical cardioversion has been linked to recurrence indexes [7]. Efficacy of certain drug therapies such as ibutilde have also been related to atrial organization [10]. Consequently, characterization of the degree of organization of the atria as a measurement of the degree of electrical remodeling appears to be essential for the selection of the best treatment for each patient. 



\section{Chapter 3}

\section{Materials}

\subsection{PTB database}

3.2

BSPM

recordings

The database and materials employed in this thesis are described in this section. This includes a public database, the Physikalisch-Technische Bundesanstalt (PTB) database which contains simultaneous standard ECG recordings and Frank's VCG leads and a custom database that we recorded with our BSPM system in cooperation with Hospital Clínico Universitario de Valencia, (HCUV, Valencia, Spain), Otto-von-Guericke University Hospital (OVGUH, Magdeburg, Germany) and Leipzig Heart Center (LHC, Germany).

We used PTB database for testing the accuracy in derivation of orthogonal leads from the standard electrocardiogram both during sinus rhythm and during atrial fibrillation. We additionally used the PTB database for studying spatial characteristics of atrial fibrillatory waves relative to the suitability of VCG systems in the analysis of atrial fibrillation.

We used our BSPM database for comparing different methods for computing optimum lead sets specific to a given clinical condition and then for determining the optimum lead set for the study of AF. In a second step, we used our BSPM database for constructing wavefront propagation maps and quantifying the degree of organization of atrial fibrillation in each patient.

\subsection{PTB database}

We used the Physikalisch-Technische Bundesanstalt (PTB) Diagnostic ECG database available on PhysioNet's webpage [40]. This database consists of 11 
simultaneously recorded signals: the conventional 12-lead ECG (with only the 8 independent leads available) and 3 Frank orthogonal leads. Signals were acquired for two minutes with 16 bit resolution over a range of $\pm 16.384 \mathrm{mV}$ and stored with a sampling frequency of $1 \mathrm{KHz}$. Figure 3.1 shows an example of the recordings from one patient.

The PTB database contains recordings from 294 subjects with different diagnoses. Diagnoses were established prior to the study phase based on annotations in the database (when available) and by visual inspection of ECG recordings by a cardiologist:

- 13 patients with atrial fibrillation.

- 1 patient with atrial flutter

- 247 without atrial arrhythmias, pacemaker implantation or AV block.

- 33 patients with other diagnosis.

\subsection{BSPM recordings}

\subsubsection{Acquisition system}

Our BSPM system consists on a commercial 64-lead recording system for biopotential measurements, Active One, Biosemi. This recording system has a bandwidth from 0 to $500 \mathrm{~Hz}$, sampling frequency of $2048 \mathrm{~Hz}$ and a quantization resolution of $1 \mu \mathrm{V} /$ bit. The system consists of a set of electrodes, battery, amplifier with an optical fiber output and a receiver that transmits the signals to a computer. The transmission via optical fiber assures isolation of the patient with the power supply, increasing the safety of the system. Electrodes used in Active One system provide unipolar measurements referred to a common electrode that can be placed at any location on the body surface. These electrodes perform a first step of amplification in the same electrode, reducing the influence of noise introduced by the wires that connect the electrodes to the amplifier and thus improving the signal quality. These active electrodes are reusable and, as opposed to disposable electrodes, they do not provide any fixation system to be attached to the body of the patient. Without any specific support system for the electrodes, attachment of 64 electrodes to the body of the patient at repeatable positions requires an excessive amount of time and/or may not assure good contact of the electrodes to the body of the patient.

Good contact of the electrodes with the skin of the patient is necessary for achieving an acceptable signal quality. With the the aim at providing good contact and allow reasonable acquisition times, electrodes were mounted in a custom-made support system, patented by our group [56], which is then attached to the patient's body. A sketch of the whole system is depicted in 

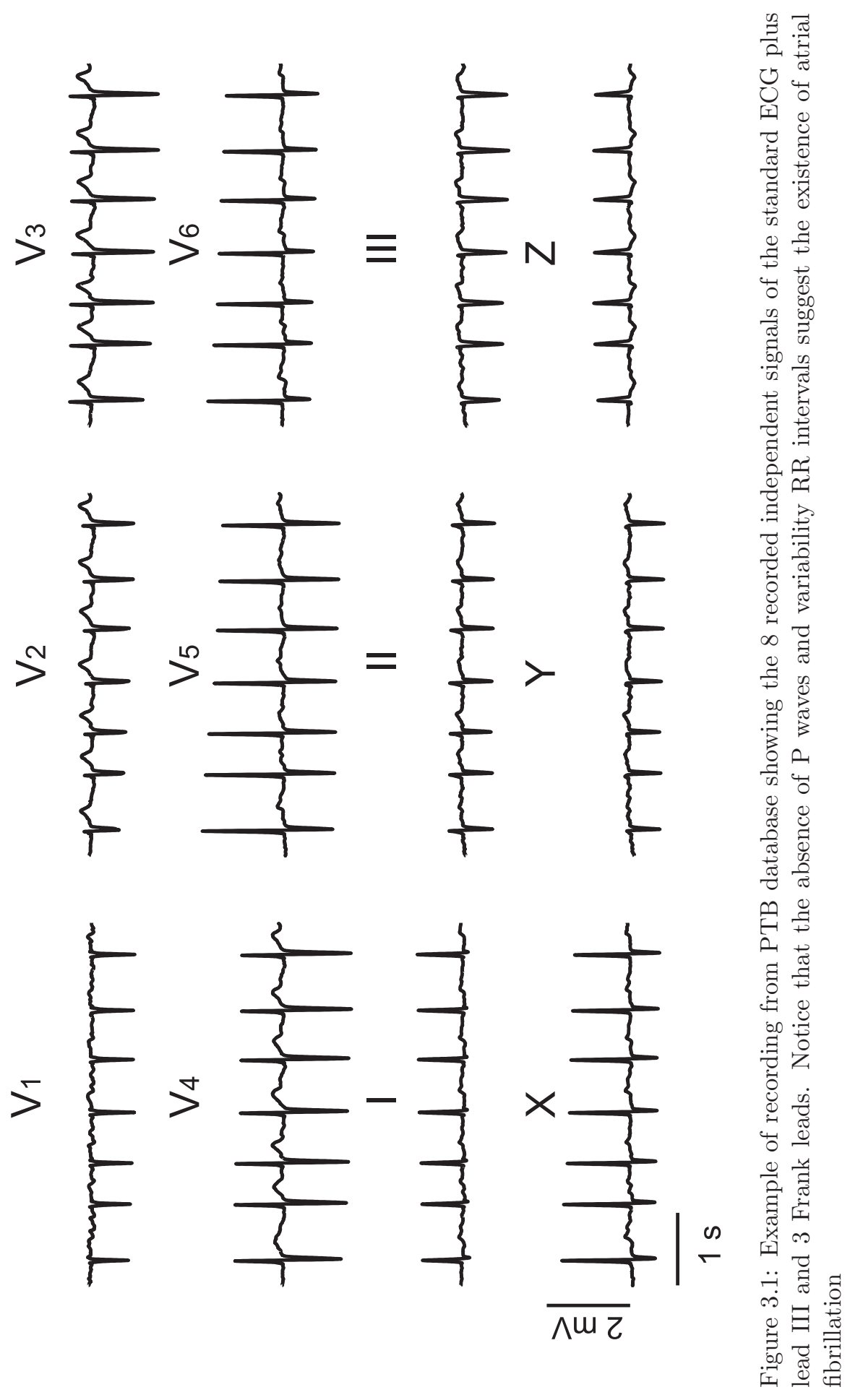
Figure 3.2. This support system was constructed in two different configurations: configuration 1 with electrodes covering the entire body surface and configuration 2 with electrodes covering the area adjacent to the atria. In both configurations perforations were applied to a supporting material: a vest in configuration 1 and a belt in configuration 2 according to the electrode grid designed. Elastic bands cover the perforations providing fixation of the electrodes and applying the needed pressure. This attachment system allows repeatability in the measurements at reasonable attachment times -under 5 minutes- and provides a good contact of the electrodes with the skin of patient, reducing movement artifacts.

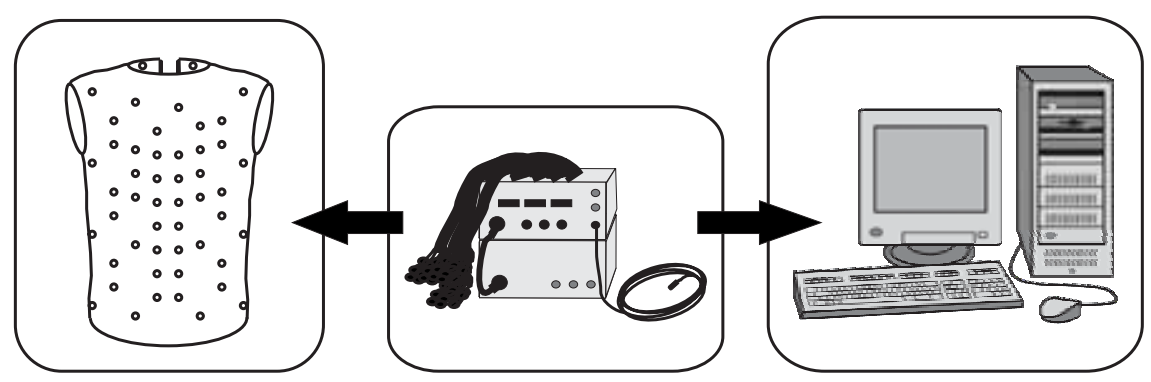

Figure 3.2: Elements constituting our BSPM system: electrode supporting system, Active One, PC

In configuration 1, a total of 64 electrodes were distributed non-uniformly upon the chest, with 16 electrodes on the back and 48 on the anterior side, with a highest density at positions overlaying the heart (see Figure 3.3). Support system for the electrodes is depicted in figure 3.4.

In configuration 2, a total of 56 chest and back leads were acquired simultaneously for each subject in addition to the standard limb leads. Chest leads $(\mathrm{N}=40)$ were arranged as a grid around $V_{1}$ with an inter-electrode distance of $2.2 \mathrm{~cm}$ while back leads $(\mathrm{N}=16)$ were arranged in a similar fashion around a lead opposite to $V_{1}\left(V_{1 \text { post }}\right)$ as depicted in Figure 3.5. Notice that the front and back parts of the belt are joined with stripes of adjustable length, adapting to the shape of the patient. In Figure 3.6 a sketch of the system is depicted.

In all cases, signal quality was visually observed during the recording by making use of the software provided by the supplier which runs under Labview 6.i. In case that an electrode showed significant noise, the contact of the electrode with the skin of the patient was revised. Figure 3.7 shows the interface of the application for the acquisition of BSPM recordings. 

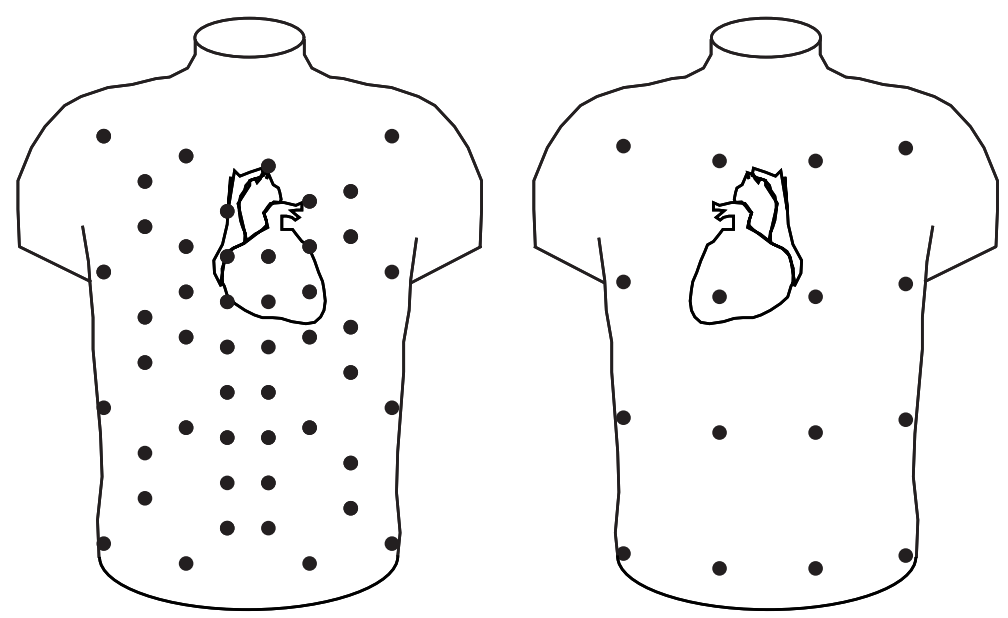

Figure 3.3: Electrode positioning of the BSPM system in configuration 1. Left: front part of the thorax. Right: back. Each dot represents an electrode position

\subsubsection{Study Population}

We acquired BSPM recordings from 151 patients with different diagnosis and using the two configurations described:

- 10-minute recordings of 17 patients with persistent atrial fibrillation using configuration 1 from OVGUH. These recordings were used for the determination of the number of the minimum number of leads and their location for the reconstruction of body surface potentials during AF (chapter 5).

- 10-minute recordings of 14 patients with persistent atrial fibrillation using configuration 2 from OVGUH $(\mathrm{N}=6)$ and LHC $(\mathrm{N}=8)$. These recordings were used for the construction of non-invasive potential maps during AF (chapter 6).

- 10-second recordings of 18 healthy volunteers using configuration 1.

- 1-minute recordings of 46 patients with an old anterior or inferior myocardial infarction (MI) using configuration 1 from HCUV.

- 1-minute recordings of 33 patients with bundle branch block (BBB) using configuration 1 from HCUV.

- 1-minute recordings of 23 patients with ventricular hypertrophy (VH) using configuration 1 from HCUV. 


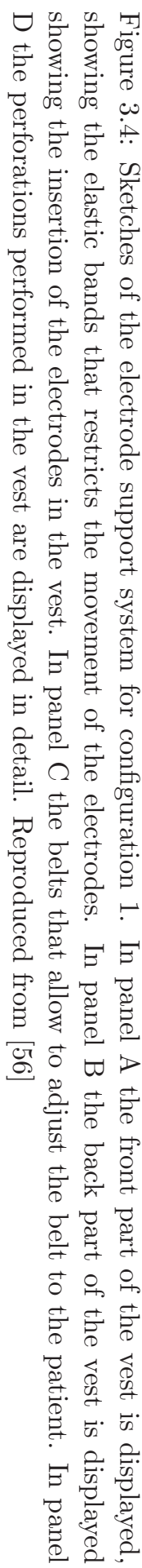

$\curvearrowright$

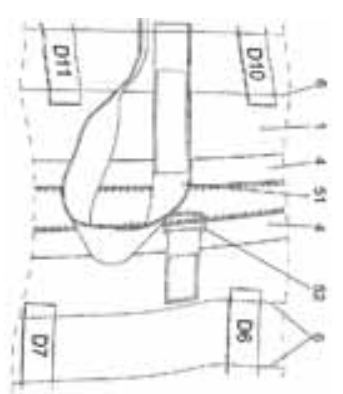

0

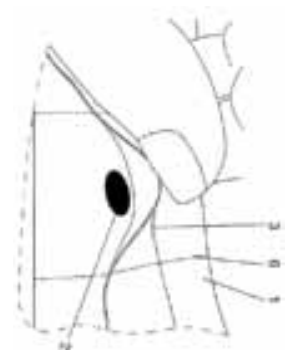

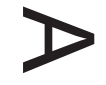

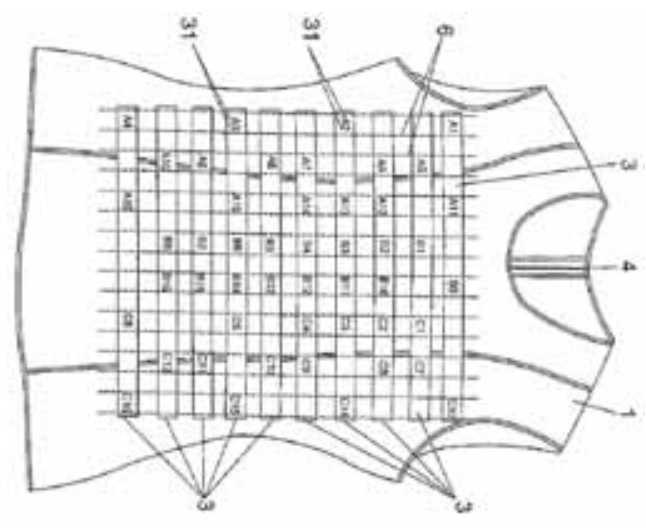

Ш

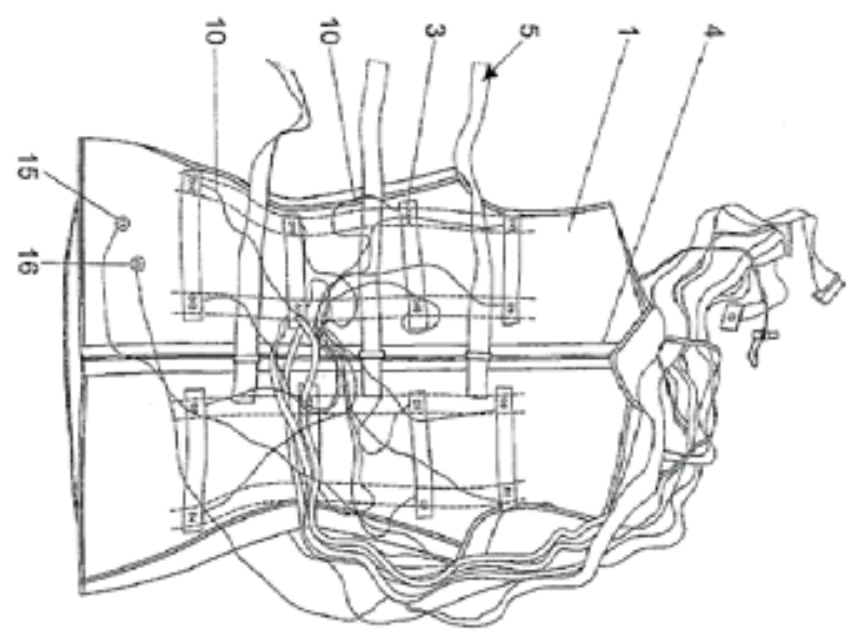




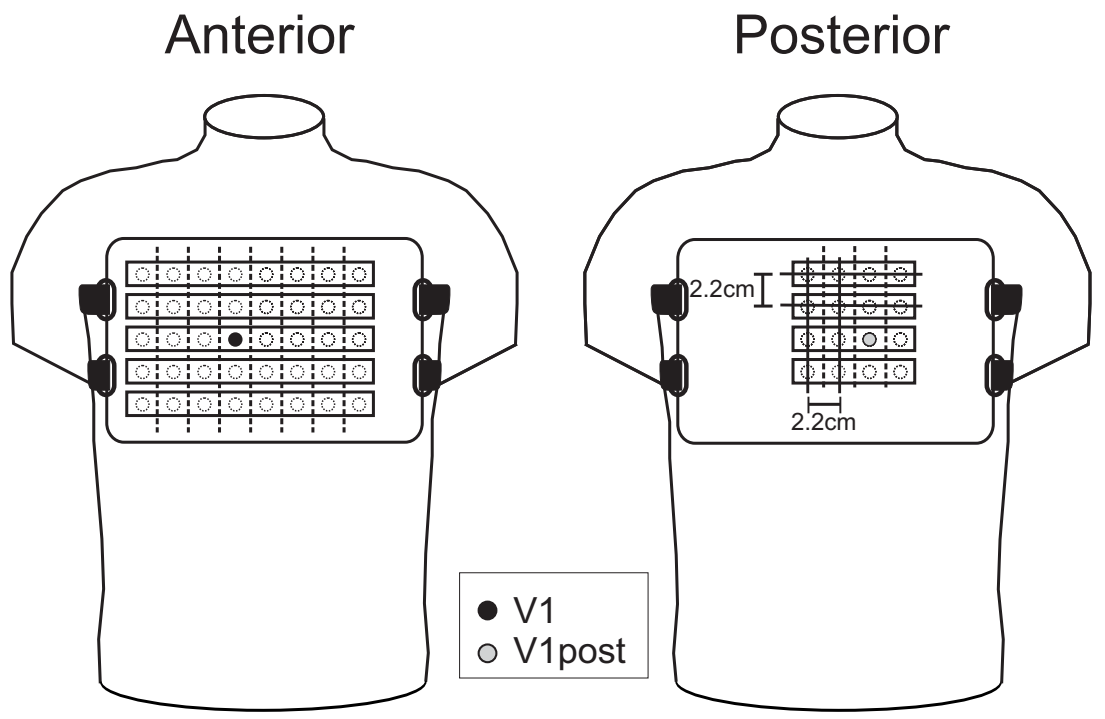

Figure 3.5: Arrangement of the electrodes and belt used for their attachment to the patient in configuration 2. Electrode positions are represented as open circles while V1 and V1post are denoted by black and grey circles respectively. Electrodes were placed around $V_{1}$ and $V_{1 \text { post }}$ as a uniform grid.

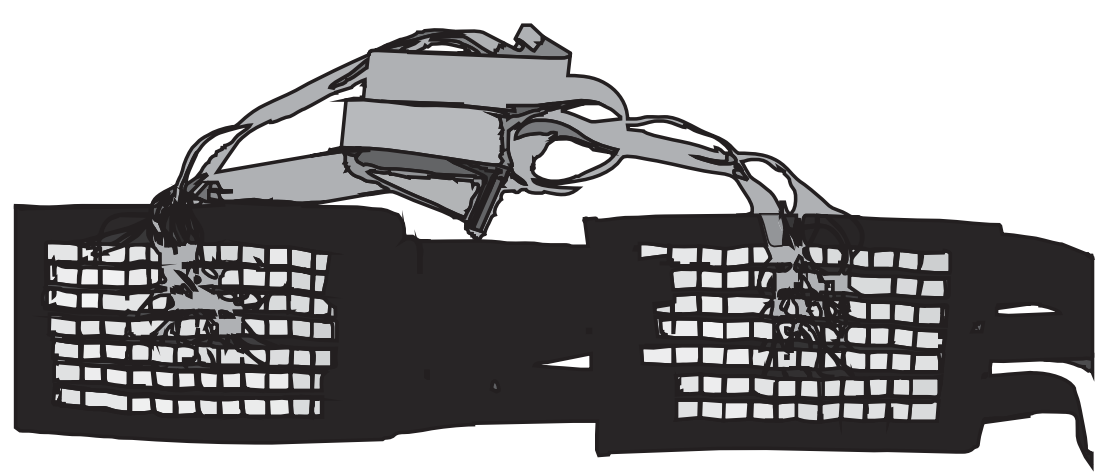

Figure 3.6: Sketch of the electrode support system for configuration 2, showing the elastic bands and belts for adjusting the system to the patient and assuring good contact of the electrodes with the skin. 


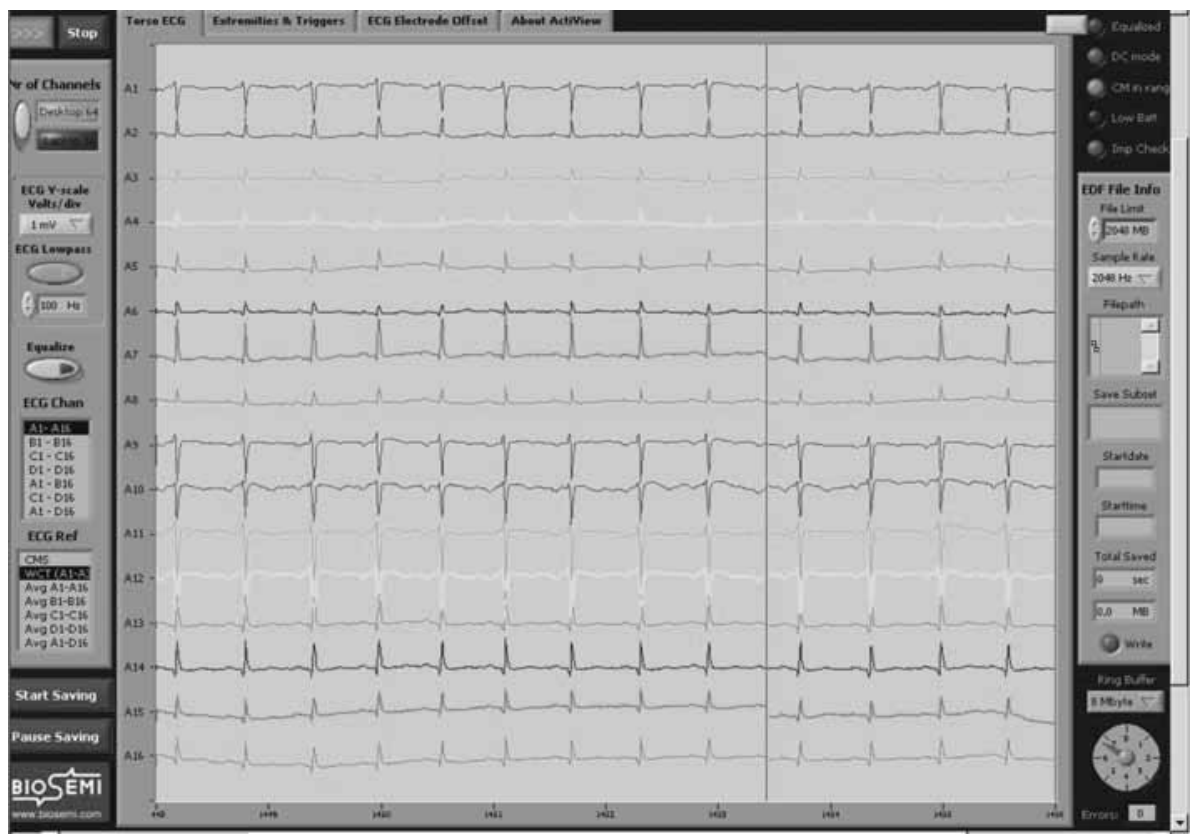

Figure 3.7: Software interface for the acquisition of BSPM recordings.

Recordings from healthy volunteers, MI, BBB and VH patients were used for comparing the performance of different lead selection methods and for computing reference performance values for the interpretation of the performance of limited lead systems in non-fibrillating rhythms (chapter $5)$.

Figure 3.9 shows an example of 64-lead recordings from a healthy subject using configuration 1. 


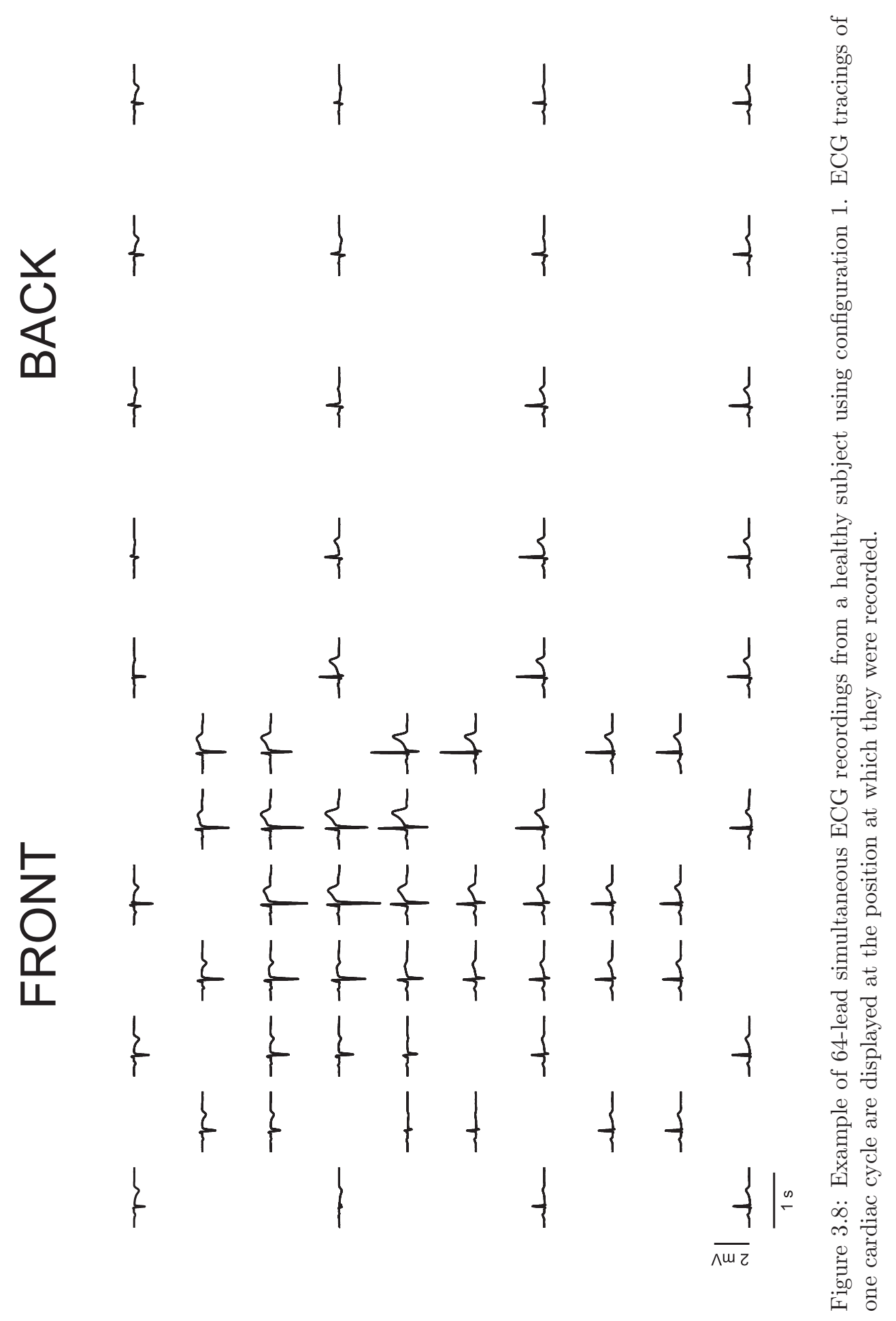




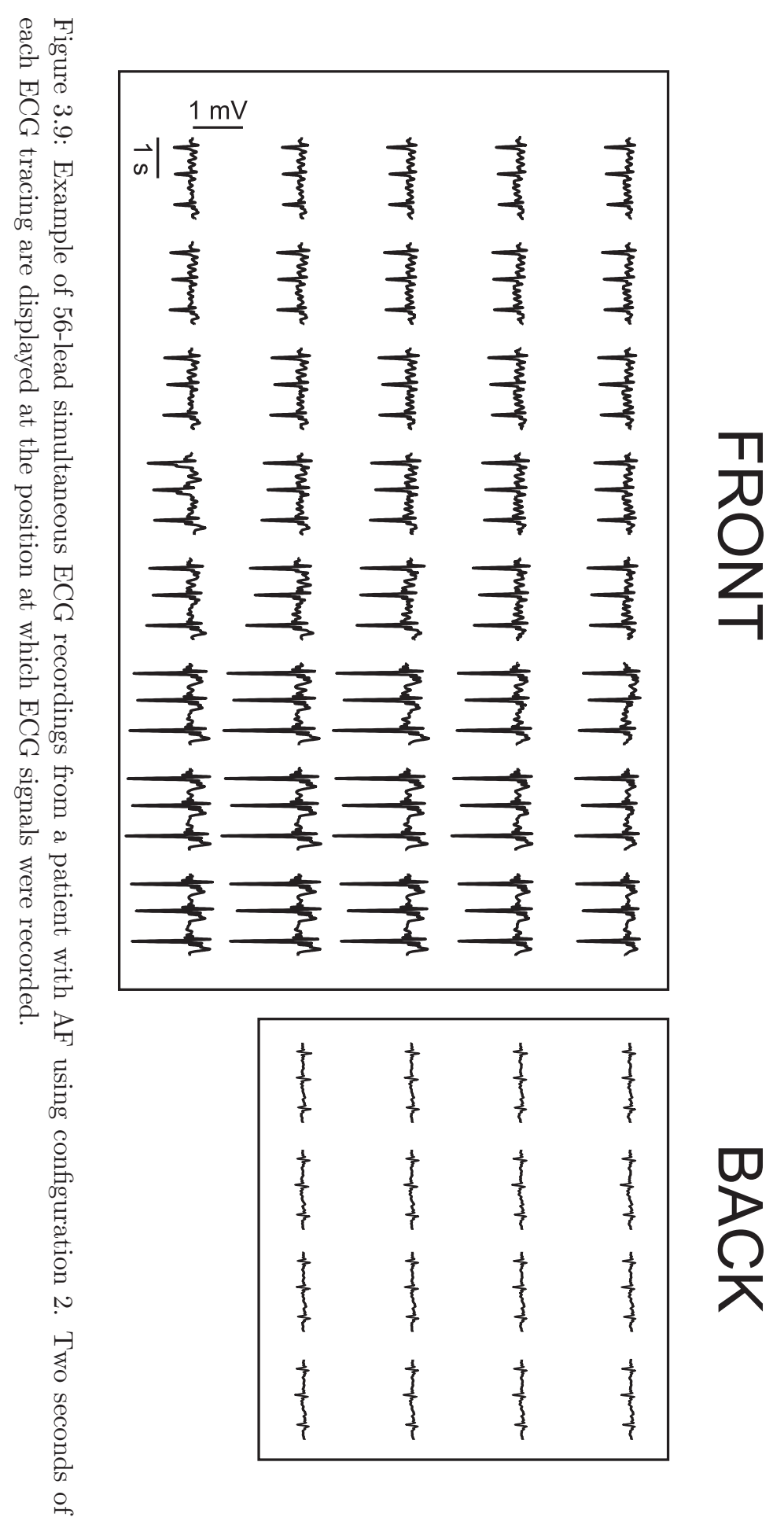




\section{Chapter 4}

\section{Spatial characteristics from the $\mathbf{E C G}^{1}$}

$\begin{array}{ll}4.1 & \text { Introduction } \\ 4.2 & \text { Materials and Methods } \\ 4.3 & \text { Results }\end{array}$

4.1 Introduction

4.3 Results

\author{
4.4 Discussion \\ 4.5 Conclusions
}

In this chapter we evaluated the possibility of determining spatial parameters of atrial activation from the standard ECG. Derivation of spatial indicators from the standard ECG is usually accomplished by the derivation of Frank's VCG. However, Frank's model established the center of coordinates in the ventricles and thus both his weighting coefficients and the relation among his orthogonal leads may be inaccurate for the atria. More importantly, derivation of nonrecorded leads from a limited number of recorded leads, that is, the standard ECG, can only be accurate in case that recorded leads do contain most electrical information. Accuracy of derivation methods based on the standard ECG have not been previously tested for the case of atrial fibrillation. In this chapter we evaluate the validity of classical derivation transforms for retrieving orthogonal leads from the standard ECG and considering both the effect of the difference in

${ }^{1}$ Chapter based on the manuscripts:

- M. S. Guillem, A. V. Sahakian and S. Swiryn. Derivation of orthogonal leads from the 12-lead electrocardiogram. Performance of an atrial-based transform for the derivation of P loops. Journal of Electrocardiology 41: 19-25, 2008.

- M. S. Guillem, A. M. Climent, A. Bollmann, D. Husser, J. Millet and F. Castells. Limitations of Dower's inverse transform for the study of atrial loops during atrial fibrillation. Pacing and Clinical Electrophysiology (Under revision). 
location of the atria with respect to the ventricles and the and the effect of the insufficient sampling of the surface of the body accomplished by the standard ECG irrespective to the location of the atria and the ventricles.

\subsection{Introduction}

The 12-lead electrocardiogram (ECG) is the most extended noninvasive technique in the diagnosis of atrial arrythmias. However, the standard ECG does not provide spatial information that can be obtained with a vectorcardiogram (VCG). The VCG allows the observation of the contribution of a specific tissue region on the morphology of $\mathrm{P}$ waves after isolation of the pulmonary veins [22] and spatial parameters derived from the VCG constitute a risk factor in post myocardial infarction patients [4]. Also, the study of the VCG during AF allows the extraction of spatial characteristics of AF waves that have been related to the organization of the atrial activity during AF [102].

Despite these advantages, the VCG is not usually recorded in clinical practice due to the inconvenience of placing additional electrodes and storage of additional signals. In case that vectorcardiographic studies are to be performed when the orthogonal leads are not recorded, these orthogonal leads need to be derived from the conventional 12 leads.

Previous studies on the VCG have made use of the so-called Dower's inverse transform for the derivation of the VCG from the 12-lead ECG $[25,28]$. Dower based the derivation of his transform coefficients on the experiments performed by Frank [42, 44], and thus assumed the hypothesis of a single stationary electrical dipole varying in direction and amplitude immersed in a homogeneous medium. The location of this stationary dipole was computed to be in the center of ventricular depolarization. Because of the simplicity of this model, the accuracy of this derivation has been previously tested for the QRS complex and compared to other derivation methods such as the Least Squares Value (LSV) optimization [81] and multiple regression analysis [77]. Although results from LSV optimization and multiple regression analysis were shown to be superior to Dower's, it is generally accepted that the degree of accuracy of Dower's transform is high enough to be considered as a satisfactory method of derivation of the QRS complex.

Although Dower's inverse transform has been tested on the QRS complex, it has been used for the derivation of atrial waves in case of AFL and AF [101, 102]. $\mathrm{Ng}$ et al. studied the orientation of atrial loops by computing the plane of best fit of AF loops and observed that some patients presented preferential orientations that were attributed to higher organization degrees. More recent studies [106] have made use of a similar approach and also used Dower's inverse transform for the derivation of $\mathrm{AF}$ loops and concluded that it is possible to relate $\mathrm{AF}$ frequency and spatial characteristics. 
However, the accuracy of Dower's inverse transform for the derivation of atrial waves is questionable. Dower based the determination of the coefficients of his transform on an ideal location of the electrical dipole corresponding to myocardial electrical activation. The location of the origin of the electrical dipole was designed with the purpose of providing good performance on ventricular depolarization. As initially pointed out by Frank [41], his determination of the center of the heart was only valid for the QRS complex as the centers of $\mathrm{P}$ and $\mathrm{T}$ activity are not the same as that of the QRS complex. Computations of transform coefficients making use of Frank's model may also not be as accurate for atrial waves than for the QRS complex. First experiments by Dower soon demonstrated that his transform was less accurate for the derivation of $\mathrm{P}$ waves that occasionally appeared with reverse polarity or changes in their morphology. For this reason, Dower changed empirically the coefficients of his transformation in order to provide better results for the derivation of $\mathrm{P}$ waves. Dower concluded that although the differences between original and derived waves would not always appear negligible to an engineer, to a cardiologist, who perceives such differences in the light of his experience with intra- and inter-individual variation and their interpretative significance, the differences appear minor $[27,26]$.

These differences, however, may result in non negligible deviations in spatial indicators specially in the case of atrial waves. This degree of inaccuracy of derived versus true recorded $\mathrm{P}$-waves was studied by Carlson et al. [16] for a database of 41 subjects. These authors concluded that $\mathrm{P}$ waves derived by Dower's transform accurately reproduced $\mathrm{P}$ wave morphology by measuring morphological parameters from the vectorcardiogram and correlation of true versus derived orthogonal leads. They observed that morphological indicators of the propensity to develop atrial fibrillation were preserved. However, they did not report any result in the accuracy of spatial indicators of derived atrial waves.

In the present work, we tested the accuracy of Dower's inverse transform for the derivation of orthogonal atrial waves from a spatial perspective. We made use of a database of 294 subjects in which simultaneous orthogonal leads and conventional 12 leads were available.

In a first step, we tested the accuracy of Dower's inverse transform in the derivation of sinus atrial waves. Also, we developed a transform optimized for retrieving atrial activity on the derivation of orthogonal leads from the 12-lead ECG and superior to Dower's inverse transform. We assumed that this new transform would offer better performance for the derivation of the P-wave than Dower's inverse transform, but worse performance for the derivation of the QRS complex. In order to test this hypothesis, we compared true recorded $\mathrm{P}$ waves versus $\mathrm{P}$ waves derived with Dower's inverse transform and our new developed transforms both for the P-wave and the QRS complex.

This P-wave optimized transform was calculated by LSV optimization 
performed on the recorded ECGs and VCGs of 123 subjects out of the 247 subjects in sinus rhythm. Once this $\mathrm{P}$-wave optimized transform was developed, and assuming that the P-wave optimized transform might be stable with independency of the individuals used for its computation, we compared its performance to the performance of a QRS complex optimized transform and to the original Dower inverse transform on a test set consisting of the remaining 124 subjects in the database.

In a second step, we investigated the suitability of Dower's inverse transform and our P-wave optimized transform for the derivation of AF loops. We made use of the ECG and VCG signals of the 13 patients in our database in which AF was present. We derived Frank leads from the 12-lead ECG by using Dower's inverse transform and compared Frank leads to derived orthogonal leads. We calculated the orientation of AF loops of both recorded orthogonal leads and derived leads and measured the difference in estimated orientation. Additionally, we attempted to link our findings to other characteristics of $\mathrm{AF}$ waves, such as amplitude, fit to a plane or $\mathrm{AF}$ frequency.

\subsection{Materials and Methods}

\subsubsection{Study Population}

We used the PTB Diagnostic ECG database available on PhysioNet's webpage. This database consists of 15 simultaneously recorded signals: the conventional 12-lead ECG and 3 Frank orthogonal leads. For a detailed description of this database see section 3.1.

The PTB database contains recordings from 294 subjects with different diagnoses. However, for purposes of our study, patients with diagnosis of AV block and/or implanted pacemakers were excluded. Those diagnoses were established prior to the study phase based on annotations in the database (when available) and by visual inspection of ECG recordings by a cardiologist blinded to the results of the study.

Patients with sinus rhythm $(\mathrm{N}=247)$ were used for the evaluation of Dower's inverse transform in the derivation of orthogonal $\mathrm{P}$ waves and for the computation transform matrices optimized for the QRS complex and $\mathrm{P}$ wave derivation. Patients with atrial fibrillation $(\mathrm{N}=13)$ were used for the evaluation of Dower's inverse transform in the derivation of atrial fibrillatory waves.

\subsubsection{Signal conditioning}

ECG signals were downloaded from PhysioNet, and read and preprocessed using algorithms implemented by our group under Matlab 7.0.1 (The Mathworks Inc, Natick, USA). 
Signals were band-pass filtered with a passing band between 0.7 and $70 \mathrm{~Hz}$ in order to reject noise and preserve ECG components [121]. Power line interference (at $50 \mathrm{~Hz}$ ) was evaluated in each lead by the Welch spectrum estimator, using a Hamming window of $8 \mathrm{~s}$, an overlap of $4 \mathrm{~s}$ and FFT with 16000 points. Leads presenting a power level in the $49-50 \mathrm{~Hz}$ band greater than $0.3 \%$ of the total power of the lead were further processed in order to eliminate this $50 \mathrm{~Hz}$ contribution. Then, QRS peaks were detected by using a modified version of Tompkins algorithm [104]. Detections were visually inspected in order to avoid incorrect detections of the algorithm.

\subsubsection{Preprocessing of $\mathrm{P}$ waves}

A representative $\mathrm{P}$ wave was computed for each lead and patient by using a template matching algorithm. Two different matching algorithms were implemented: a PQRST based matching and a $\mathrm{P}$ wave based matching. For the PQRST matching algorithm, QRS peaks were detected using a modified version of Pan and Tompkin's algorithm [104]. A window starting $350 \mathrm{~ms}$ before each QRS peak detected in lead 1 (QRSp1) and $400 \mathrm{~ms}$ after QRSp1 was selected to perform the correlation and subsequent averaging after positive matching. This window size had to be increased in 12 patients with a long PR interval. The correlation coefficient established for positive matching in the algorithm was set to 0.97 . For the $\mathrm{P}$ wave matching algorithm, correlation was performed on the $\mathrm{P}$ wave only, and the same matching threshold of 0.97 was used. After averaging, templates were low-pass filtered using a Butterworth filter with a cutoff frequency of $50 \mathrm{~Hz}$. Due to the noise level present in our signals, the number of $\mathrm{P}$ waves exceeding this correlation threshold was low, which didn't allow us to find an acceptable number of P-waves for averaging. As a consequence, $\mathrm{P}$ waves computed with a PQRST matching presented lower errors than those averaged after a $\mathrm{P}$ wave based matching and thus we decided to use waves averaged by using the PQRST based template matching algorithm. We based our decision on the assumption that P-wave morphology would remain similar for the time interval studied (2 minutes). Also, accepting that in some patients this assumption could be incorrect, we preferred the inaccuracy due to $\mathrm{P}$-wave changes during 2 minutes to the level of noise present when a low number of $\mathrm{P}$-waves is used for averaging.

A step prior to the analysis of individual waves in the ECG is the delineation of QRS and $\mathrm{P}$ wave onset and offset points. Onset and offset points were automatically detected for each lead among local minima of a function that estimates the radius of curvature, as described in [21]. After automatic wave detection, fiducial points were displayed, resulting in an incorrect detection of either $\mathrm{P}_{\text {onset }}$ or $\mathrm{P}_{\text {offset }}$ in 46 patients and incorrect $\mathrm{QRS}_{\text {onset }}$ or $\mathrm{QRS}_{\text {offset }}$ in 37 patients, for which fiducial points were edited manually. 


\subsubsection{Preprocessing of AF waves}

Spatial analysis was performed in AF segments free from ventricular activity (TQ segments) and with duration equal to the dominant atrial cycle length (DACL). Initial and final points of the QRS complex $\left(\mathrm{QRS}_{\text {onset }}, \mathrm{QRS}_{\text {offset }}\right)$ and $\mathrm{T}$ wave $\left(\mathrm{T}_{\text {offset }}\right)$ were detected and visually inspected. AF segments free from ventricular activity were defined as starting $25 \mathrm{~ms}$ after the last ( $\mathrm{T}_{\text {offset }}$ ) found in the 15 leads and ending $25 \mathrm{~ms}$ before the first $\left(\mathrm{QRS}_{\text {onset }}\right)$ detected. Only AF segments longer than $250 \mathrm{~ms}$ were considered for our study, whereas shorter segments were discarded. AF segments were again band-pass filtered between 2 and $30 \mathrm{~Hz}$ in order to reject noise components that do not correspond to the spectral content of AF [122].

In order to obtain the DACL, atrial fibrillatory signals were estimated from the surface ECG by cancelling the ventricular activity from the subtraction of a matching QRS-T template. This template was computed for each beat as a linear combination of the principal components previously obtained from the analysis of all beats in the ECG segment [18]. Welch's periodogram was used to obtain the power spectral density of atrial signals (hamming window of 5.12 seconds and $50 \%$ overlap). The dominant frequency $\left(\mathrm{F}_{\mathrm{AA}}\right)$ was defined as the peak with higher spectral density in the power spectrum. The DACL was calculated as the inverse of the dominant frequency found in lead $\mathrm{V}_{1}$. Filtered AF segments were split into shorter segments with a length equal to the DACL.

\subsubsection{Optimized transforms for P-wave and QRS complex derivation}

Half of the patients in sinus rhythm in the database (every other patient among those not previously excluded, $\mathrm{N}=124$ ) were selected as a study set for the computation of optimal matrices for the QRS complex and the P-wave.

Transform matrices were computed by making use of recorded Frank leads:

$$
\mathbf{V C G F}=\left[\begin{array}{lll}
\mathbf{x}_{F} & \mathbf{y}_{F} & \mathbf{z}_{F}
\end{array}\right]^{T}
$$

And the eight independent standard ECG leads

$$
\mathbf{E C G 8}=\left[\begin{array}{lllllll}
\mathrm{V}_{1} & \mathrm{~V}_{2} & \mathrm{~V}_{3} & \mathrm{~V}_{4} & \mathrm{~V}_{5} & \mathrm{~V}_{6} & \mathrm{I} \\
\mathrm{II}
\end{array}\right]^{\mathrm{T}}
$$

For each patient, an individual transform matrix optimized for the recovery of the $\mathrm{P}$ wave $\left(P L S V_{i}\right)$ was obtained by applying LSV optimization in $V C G F$ and $E C G 8$ matrices over the time interval included between the $P_{\text {onset }}$ and the Poffset .

$$
\mathrm{PLSV}_{i}=\mathrm{VCGF}_{\left\{\mathrm{P}_{\mathrm{onset}} \ldots \mathrm{P}_{\mathrm{offset}}\right\}} \cdot \mathbf{E C G}_{\left\{\mathrm{P}_{\mathrm{onset}}^{\dagger} \ldots \mathrm{P}_{\mathrm{offset}}\right\}}^{\dagger},
$$

where ${ }^{\dagger}$ denotes the Moore-Penrose pseudo-inverse of a matrix and $i$ stands for the number of the individual patient evaluated. In the same fashion, an 
individual transform matrix was obtained for the QRS complex $\left(\mathbf{Q L S V} \mathbf{V}_{i}\right)$ by solving the coefficients of the transformation by the least squares method on the QRS interval.

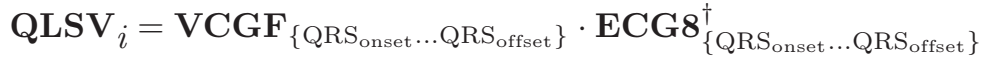

The optimal transform matrix for the recovery of the $\mathrm{P}$ wave (PLSV) was calculated as the average of all $\mathbf{P} \mathbf{L} \mathbf{S} \mathbf{V}_{i}$ s matrices.

$$
\mathbf{P L S V}=\frac{1}{\mathrm{~N}} \cdot \sum_{i=1}^{\mathrm{N}} \mathbf{P L S V}_{i},
$$

where $\mathrm{N}$ is the number of patients.

The optimal transform for the recovery of the QRS complex (QLSV) was computed as the mean of all $\mathbf{Q L S} \mathbf{V}_{i}$ s matrices.

$$
\mathbf{Q L S V}=\frac{1}{\mathrm{~N}} \cdot \sum_{i=1}^{\mathrm{N}} \mathbf{Q L S V}_{i}
$$

\subsubsection{Stability of transform matrices}

In order to assess the stability of the optimized transforms with the population included in the computation of the matrix, we calculated the optimized PLSV and $\mathbf{Q L S V}$ transforms for the test set $\left(\mathbf{P L S V} \mathbf{V}_{\mathrm{TEST}}\right)$ and $\left(\mathbf{Q L S V} \mathbf{V}_{\mathrm{TEST}}\right)$. These transform matrices were not used for the derivation of orthogonal leads on the test set. However, we evaluated the resemblance of those transform matrices calculated with different patient sets by computing the correlation among coefficients of the different transforms.

\subsubsection{Derivation of orthogonal leads from the ECG}

Orthogonal leads were derived from the standard ECG by making use or Dower's inverse transform, PLSV and QLSV transforms:

$$
\begin{aligned}
\text { VCGD } & =\mathrm{D}^{-1} \cdot \mathbf{E C G 8} \\
\text { VCGP } & =\mathrm{PLSV} \cdot \mathbf{E C G 8} \\
\text { VCGQ } & =\mathrm{QLSV} \cdot \mathbf{E C G 8}
\end{aligned}
$$

\subsubsection{Performance on the $\mathrm{P}$ wave and QRS complex}

Patients not included in the study set constituted the test set $(N=123)$. For every patient in the test set, derived cardiac cycles of the XYZ leads obtained 
by the three studied optimal transforms were compared with true recorded XYZ leads. In order to compare the accuracy of the recovery, we measured for each lead (X, Y and Z), derivation method (Dower, QLSV and PLSV) and wave (P and QRS), the correlation coefficients between derived and true measured waves for each lead and the root mean squared error. We define the correlation index between recorded and derived $\mathrm{x}$ lead on the $\mathrm{P}$ wave:

$$
\mathrm{R}_{\mathrm{x}}=\frac{n \sum_{i} \mathbf{x}_{\mathrm{d}}(i) \cdot \mathbf{x}_{\mathrm{F}}(i)-\sum_{i} \mathbf{x}_{\mathrm{F}}(\mathrm{i}) \cdot \sum_{i} \mathbf{x}_{\mathrm{d}}(i)}{\sqrt{n \sum_{i} \mathbf{x}_{\mathrm{F}}(i)^{2}-\left(\sum_{i} \mathbf{x}_{\mathrm{F}}(i)\right)^{2}} \cdot \sqrt{n \sum_{i} \mathbf{x}_{\mathrm{d}}(i)^{2}-\left(\sum_{i} \mathbf{x}_{\mathrm{d}}(i)\right)^{2}}}
$$

Where $i=\mathrm{P}_{\text {onset }} \ldots \mathrm{P}_{\text {offset }}, \mathbf{x}_{F}$ stands for recorded lead $\mathbf{x}, \mathbf{x}_{d}$ for derived orthogonal lead $\mathrm{x}$ and $n$ is the number of sample points between $\mathrm{P}_{\text {onset }}$ and $\mathrm{P}_{\text {offset }}$. Correlation indexes were computed for all leads $\left(\mathrm{R}_{\mathrm{x}}, \mathrm{R}_{\mathrm{y}}, \mathrm{R}_{\mathrm{z}}\right)$, derivation methods and wave ( $\mathrm{P}$ wave and QRS complex).

We define the Root Mean Squared (RMS) error for derived $\mathrm{x}$ lead on the $\mathrm{P}$ wave as follows:

$$
\varepsilon=\sqrt{\frac{\sum_{i}\left(\mathbf{x}_{\mathrm{F}}(i)-\mathbf{x}_{\mathrm{d}}(i)\right)^{2}}{n}}
$$

Also, we computed the mean squared errors for leads y and $\mathrm{z}$ and of derived VCG loops. We define the instant error in derived waves:

$$
\mathbf{e}_{i}=\|\mathbf{V C G D}(i)-\mathbf{V C G F}(i)\|
$$

Being VCGD the orthogonal leads derived by any of the derivation methods specified in the previous section.

RMS error in derivation of orthogonal leads was computed as follows:

$$
\varepsilon=\sqrt{\frac{\sum_{i=1}^{N} \mathbf{e}(i)^{2}}{N}},
$$

where $\mathrm{N}$ stands for the number of samples of the wave under consideration.

Mean errors and correlations obtained with the different methods were compared with paired t-tests.

\subsubsection{Performance on AF waves}

We computed RMS error in derived waves as defined in equation 4.13. Also, we computed the amplitude of $\mathrm{AF}$ waves:

$$
A=\sqrt{\frac{\sum_{i=1}^{N}\|\operatorname{VCGF}(i)\|^{2}}{N}},
$$


where $\mathrm{N}$ is again the number of sample points in each $\mathrm{AF}$ wave. Relative error was defined as:

$$
\varepsilon_{R}=\frac{\varepsilon}{A}
$$

Errors in estimation of orientation of AF waves were also computed. Planes of best fit of each AF segment were computed by linear regression both for Frank orthogonal leads and for derived orthogonal leads. The fit to the plane was measured as the coefficient of determination $R^{2}$, which quantifies the amount of variation in the points in the VCG loop that can be explained by the plane of best fit. Orientation of an AF segment was determined by the direction of the normal vector to the plane of best fit. Error in the estimation of the orientation of an $\mathrm{AF}$ segment $\left(\varepsilon_{D}\right)$ was computed as the angle between the directions of the normal vectors to the planes computed for derived $\left(\mathbf{n}_{d}\right)$ versus non-derived $\left(\mathbf{n}_{F}\right)$ orthogonal waves:

$$
\varepsilon_{D}=\arccos \left[\frac{\left|\left\langle\mathbf{n}_{F}, \mathbf{n}_{d}\right\rangle\right|}{\left\|\mathbf{n}_{F}\right\| \cdot\left\|\mathbf{n}_{d}\right\|}\right]
$$

Notice that $\varepsilon_{D}$ ranges from 0 to 90 degrees. Wave residuum was computed by eigenvalue decomposition:

$$
r e s=\frac{\sum_{i=4}^{8} \lambda_{i}}{\sum_{i=1}^{8} \lambda_{i}}
$$

Where $\lambda_{i}$ stands for the $i$-th eigenvalue of the autocorrelation matrix of the eight independent components of the standard ECG. The first three eigenvectors resulting from eigenvalue decomposition represent the dipolar components of the ECG while $5^{\text {th }}$ to $8^{\text {th }}$ components represent the non dipolar content of AF signals. When res is close to 0, the ECG signal within this segment is mainly the result of a dipolar activation as it can be represented in a three-dimensional space. The higher the value of res, the less dipolar the ECG within the studied segment is.

In order to obtain descriptive statistics of the parameters measured, the mean value of each parameter was calculated for each patient. Then, mean and standard deviation values were obtained.

Correlation of the mean values of $\varepsilon_{R}$ and $\varepsilon_{D}$ of each patient with $F_{A A}$ was computed as the Pearson correlation coefficient. Correlation among calculated errors $\left(\varepsilon_{R}\right.$ and $\left.\varepsilon_{D}\right)$ of individual waves and other parameters measured ( $A$, res, $R^{2}$ ) was also computed aiming at determining whether a higher error is related to a low amplitude of AF waves, high residuum or poor plane fit. In this case, the same number of waves of each patient (23 waves per patient, total: 299 waves) were taken and Pearson correlation value was obtained. In both cases, a $\mathrm{p}$ value $<0.05$ was considered to be statistically significant. 


\subsection{Results}

\subsubsection{Transform matrices}

Computed PLSV and QLSV transform coefficient matrices were:

$$
\begin{aligned}
& \mathbf{P L S V}=\left(\begin{array}{rrrrrrrr}
-0.266 & 0.027 & 0.065 & 0.131 & 0.203 & 0.220 & 0.370 & -0.154 \\
0.088 & -0.088 & 0.003 & 0.042 & 0.047 & 0.067 & -0.131 & 0.717 \\
-0.319 & -0.198 & -0.167 & -0.099 & 0.009 & 0.060 & 0.184 & -0.114
\end{array}\right) \\
& \text { QLSV }=\left(\begin{array}{rrrrrrrr}
-0.147 & -0.058 & 0.037 & 0.139 & 0.232 & 0.226 & 0.199 & -0.018 \\
0.023 & -0.085 & -0.003 & 0.033 & 0.060 & 0.146 & -0.146 & 0.503 \\
-0.184 & -0.163 & -0.190 & -0.119 & -0.023 & 0.043 & 0.085 & -0.130
\end{array}\right)
\end{aligned}
$$

\subsubsection{Stability of transform matrices}

As it can be observed in Table 4.1, PLSV matrix showed a correlation of 0.99

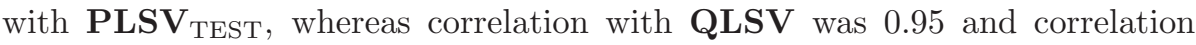
with Dower's inverse transform was 0.89. QLSV matrix showed a correlation of 0.99 with QLSV $_{\text {TEST }}$, whereas correlation with Dower's inverse transform was 0.91 .

\begin{tabular}{|c|c|c|c|c|}
\hline & PLSV & QLSV & PLSV $_{\text {TEST }}$ & QLSV $_{\text {TEST }}$ \\
\hline DOWER & 0.89 & 0.91 & 0.90 & 0.90 \\
\hline PLSV & & 0.95 & 0.99 & 0.95 \\
\hline QLSV & & & 0.95 & 0.99 \\
\hline PLSV $_{\text {TEST }}$ & & & & 0.96 \\
\hline
\end{tabular}

Table 4.1: Comparison of the different transform matrices. Correlation values between the coefficients of the matrices used: PLSV, QLSV and Dower Inverse with matrices calculated for the test set. $(p<0.001$ in all cases $)$

\subsubsection{Comparison of true vs. derived $P$ waves}

For each patient in the test group, orthogonal leads were derived using the transform matrices already described. Results of this derivation for one patient can be observed in Figure 4.1. It is noticeable leads derived with Dower's inverse transform differ from true measured orthogonal leads and especially for Z lead. Similar results can be observed for the derived loops reconstructed from the orthogonal leads for both the $\mathrm{P}$ wave (see Figure 4.2) and the QRS complex (see Figure 4.3). 

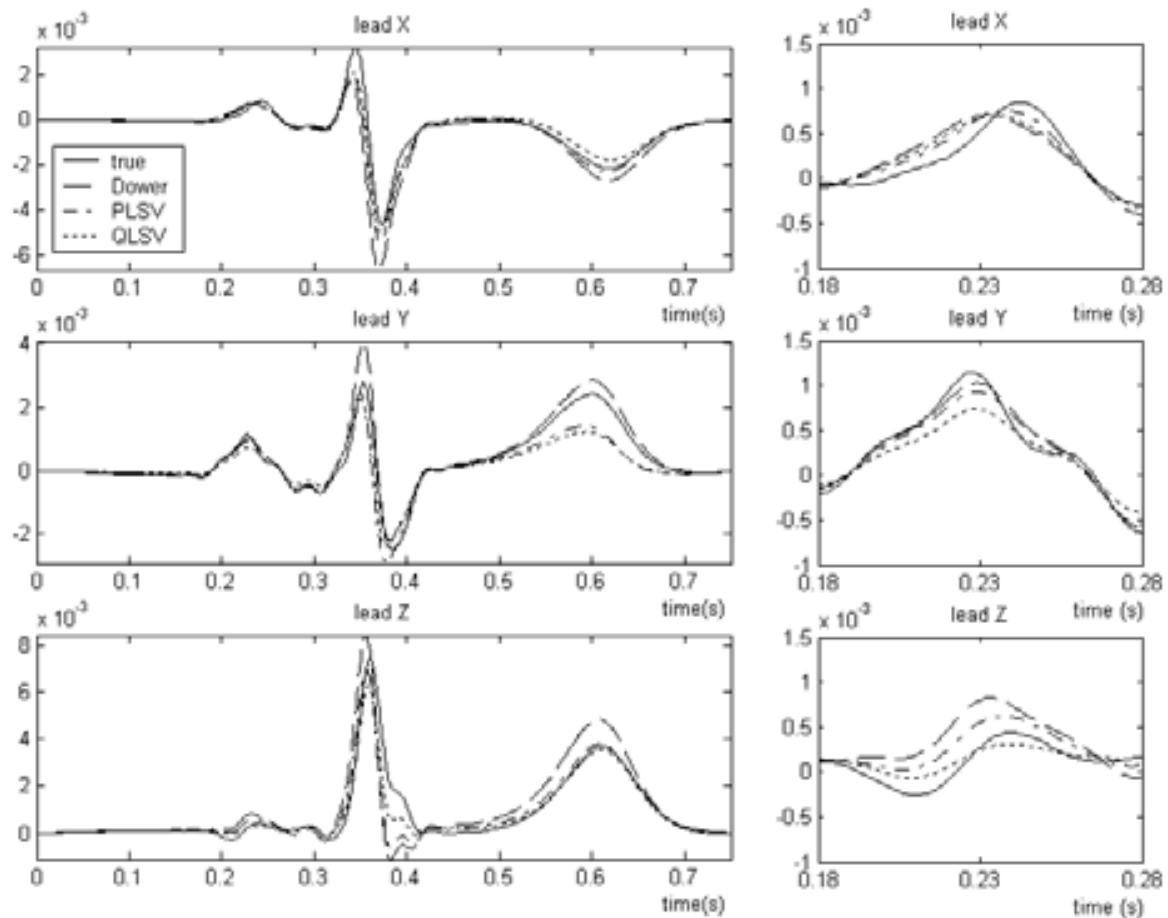

Figure 4.1: True versus derived ECGs with Dower, PLSV and QLSV transforms of patient 2. On the right, only $\mathrm{P}$-waves of the orthogonal leads are displayed for the same patient. Note that the scale of the vertical axes have been changed. 
P wave

\section{Transverse}
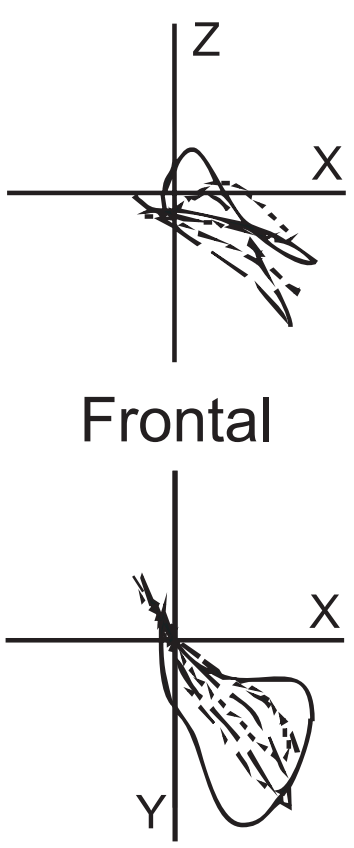

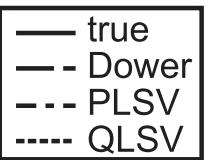

Sagital

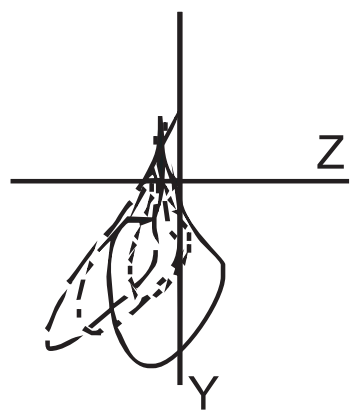

Figure 4.2: True versus derived VCGs corresponding to the $\mathrm{P}$ wave with Dower, PLSV and QLSV transforms of patient 2. 


\section{QRS complex}

\section{Transverse}

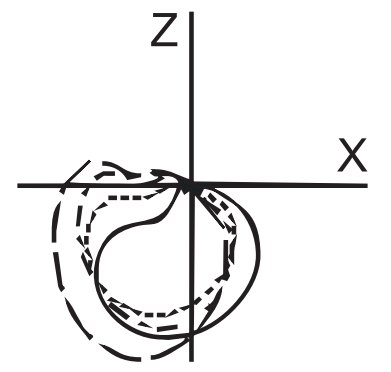

Frontal

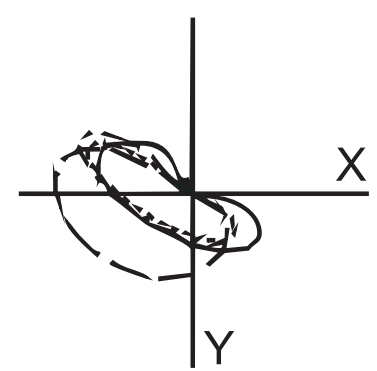

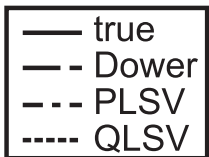

Sagital

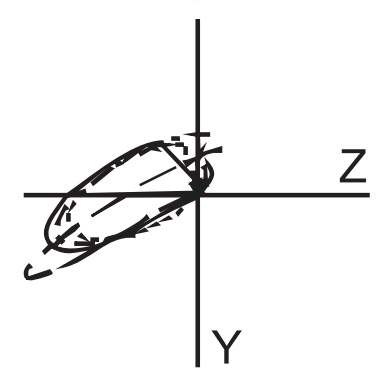

Figure 4.3: True versus derived VCGs corresponding to the QRS complex with Dower, PLSV and QLSV transforms of patient 2. 
Average results for the test set on the $\mathrm{P}$ wave can be observed in Figures 4.4-4.6 and Tables 4.2-4.4. The PLSV transform globally performed better than Dower's and QLSV for the derivation of the P wave (see Figure 4), with a mean loop root squared error of 12.2 $\mu$ VRMS vs. Dower: $14.4 \mu$ VRMS, $(\mathrm{p}<0.01)$ and QLSV: $16.0 \mu$ VRMS $(\mathrm{p}<0.01)$. Root mean squared errors were lower for leads $\mathrm{X}$ and $\mathrm{Z}(\mathrm{p}<0.01)$ and higher for lead $\mathrm{Y}(\mathrm{p}<0.01)$. Correlation values of true vs derived P-waves showed a similar behavior: performance on leads $\mathrm{X}$ and $\mathrm{Y}$ was also higher for PLSV than for both Dower's and QLSV $(\mathrm{p}<0.01)$ and similar for lead $\mathrm{Y}(\mathrm{p}<0.05)$.

\section{P wave}

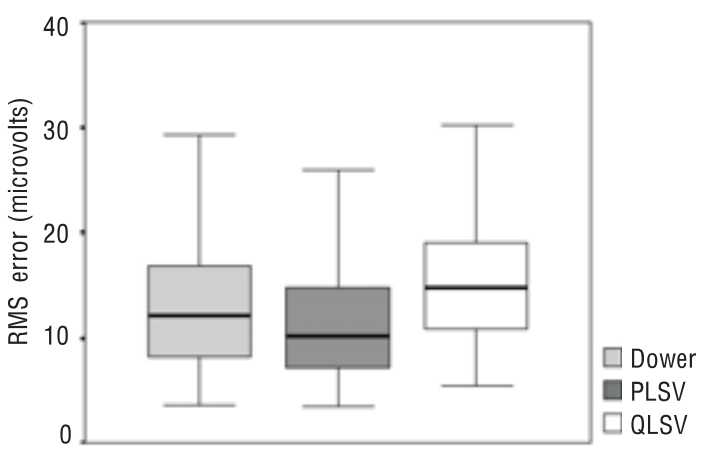

Figure 4.4: RMS error of derived versus true measured P-loops using Dower, PLSV and QLSV transforms

\begin{tabular}{|c|c|c|c|}
\hline & $\begin{array}{c}\text { Dower } \\
\text { Mean } \pm \text { SD }\end{array}$ & $\begin{array}{c}\text { PLSV } \\
\text { Mean } \pm \text { SD }\end{array}$ & $\begin{array}{c}\text { QLSV } \\
\text { Mean } \pm \text { SD }\end{array}$ \\
\hline RMS P & $14.4 \pm 9.5$ & $12.2 \pm 7.96$ & $16.0 \pm 7.38$ \\
\hline RMS Q & $121.3 \pm 65.8$ & $91.1 \pm 43.8$ & $84.8 \pm 43.8$ \\
\hline
\end{tabular}

Table 4.2: Comparison of true vs. derived orthogonal loops using Dower's, PLSV and QLSV transforms. Values represent the root mean squared error of the loop in microvolts.

In the derivation of the QRS complex from the 12-lead ECG, QLSV globally performed better than both PLSV and Dower (see Figures 4.7-4.9 and Tables 4.2-4.4, with a mean loop error of $84.8 \mu$ VRMS vs. Dower: 121.3 $\mu$ VRMS $(\mathrm{p}<0.01)$ and PLSV: $91.1 \mu$ VRMS $(\mathrm{p}<0.05)$. Root mean squared errors per lead and correlation values (true vs. derived QRS complexes) also demonstrated better performance of the QLSV transform for the derivation of the QRS complex than Dower's $(\mathrm{p}<0.01)$ and PLSV $(\mathrm{p}<0.05)$ with higher 


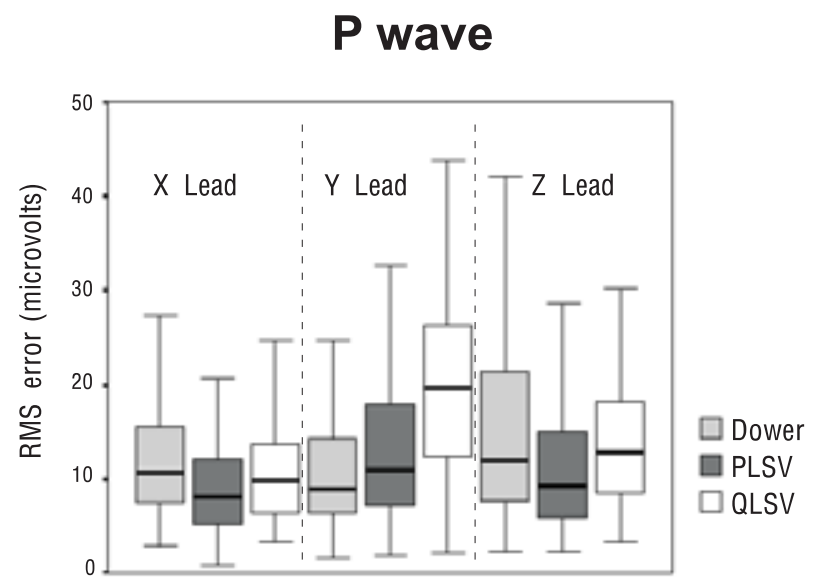

Figure 4.5: Root mean squared error of derived versus true measured $\mathrm{P}$ waves in the three orthogonal leads X,Y,Z using Dower, PLSV and QLSV transforms

P wave

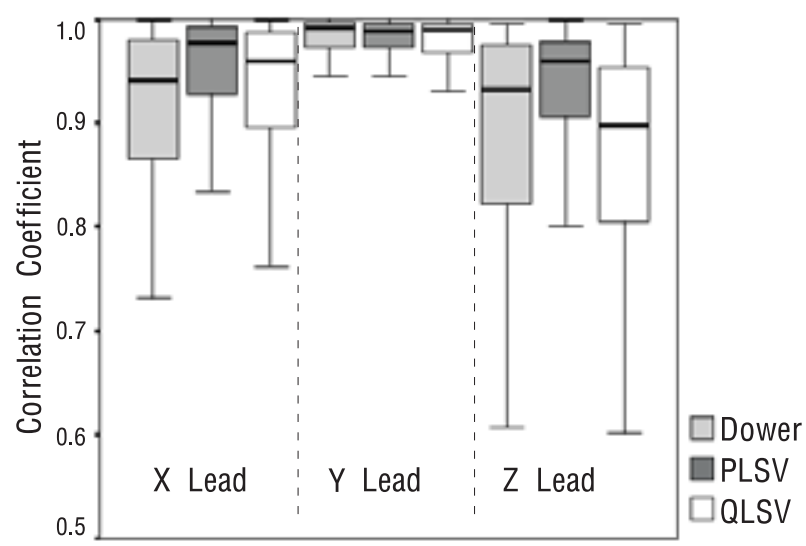

Figure 4.6: Correlation of derived vs. true measured P-waves. Correlation coefficients for Dower, PLSV and QLSV transforms 


\begin{tabular}{|c|c|c|c|c|}
\hline \multicolumn{2}{|c|}{} & Dower & PLSV & QLSV \\
\hline \multirow{3}{*}{$\mathrm{P}$} & $\mathrm{X}$ & $12.5 \pm 7.0$ & $9.3 \pm 5.6$ & $11.3 \pm 6.5$ \\
\cline { 2 - 5 } & $\mathrm{Y}$ & $13.8 \pm 16.7$ & $15.3 \pm 15.4$ & $21.9 \pm 14.7$ \\
\cline { 2 - 5 } & $\mathrm{Z}$ & $16.7 \pm 13.6$ & $11.8 \pm 8.6$ & $14.4 \pm 8.2$ \\
\hline \multirow{3}{*}{ QRS } & $\mathrm{X}$ & $96.3 \pm 77.8$ & $77.2 \pm 65.9$ & $70.4 \pm 61.2$ \\
\cline { 2 - 5 } & $\mathrm{Y}$ & $70.3 \pm 68.1$ & $72.8 \pm 53.2$ & $81.5 \pm 56.2$ \\
\cline { 2 - 5 } & $\mathrm{Z}$ & $194.4 \pm 118.9$ & $121.2 \pm 73.2$ & $100.7 \pm 67.7$ \\
\hline
\end{tabular}

Table 4.3: Comparison of true vs. derived orthogonal leads using Dower's, PLSV and QLSV transforms. Root mean squared errors in the derivation of P-waves and QRS complexes by using the three different transforms, given as mean \pm standard deviation in microvolts.

\begin{tabular}{|c|c|c|c|c|}
\hline \multicolumn{2}{|c|}{} & Dower & PLSV & QLSV \\
\hline \multirow{3}{*}{$\mathrm{P}$} & $\mathrm{X}$ & $0.88 \pm 0.15$ & $0.93 \pm 0.12$ & $0.91 \pm 0.14$ \\
\cline { 2 - 5 } & $\mathrm{Y}$ & $0.91 \pm 0.26$ & $0.90 \pm 0.27$ & $0.91 \pm 0.26$ \\
\cline { 2 - 5 } & $\mathrm{Z}$ & $0.85 \pm 0.23$ & $0.91 \pm 0.18$ & $0.84 \pm 0.20$ \\
\hline \multirow{3}{*}{ QRS } & $\mathrm{X}$ & $0.94 \pm 0.11$ & $0.97 \pm 0.06$ & $0.97 \pm 0.06$ \\
\cline { 2 - 5 } & $\mathrm{Y}$ & $0.91 \pm 0.21$ & $0.88 \pm 0.27$ & $0.86 \pm 0.28$ \\
\cline { 2 - 5 } & $\mathrm{Z}$ & $0.84 \pm 0.29$ & $0.88 \pm 0.26$ & $0.92 \pm 0.19$ \\
\hline
\end{tabular}

Table 4.4: Comparison of true vs. derived orthogonal leads using Dower's, PLSV and QLSV transforms. Correlation values in the derivation of P-waves and QRS complexes by using the three different transforms, given as mean \pm standard deviation. 
correlations and lower errors for the $\mathrm{X}$ and $\mathrm{Z}$ leads and lower correlations and higher errors for the Y lead.

\section{QRS complex}

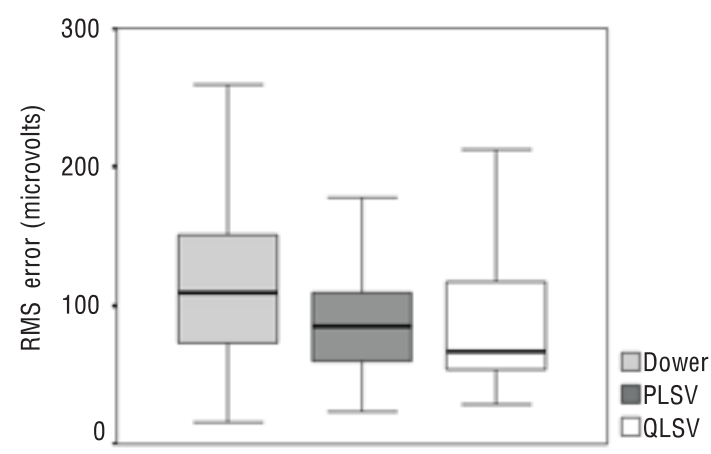

Figure 4.7: RMS error of derived versus true measured QRS-loops. Root mean squared errors for Dower, PLSV and QLSV transforms

\subsubsection{Comparison of true vs. derived AF waves}

Results obtained from our study are presented in Table 4.5. Mean residuum index (res) was found to be equal to $0.02 \pm 0.01$, meaning that $98 \%$ of the content of the signal can be represented in a three dimensional space. However, the mean fit to the plane $\left(R^{2}\right)$ was equal to 0.56 . This $R^{2}$ value represents a poor fit to the plane, as it is depicted in Figure 4.10. Although some waves can present a good fit to a plane (see Figure 4.10.A-B), other waves can present a very poor fitting (see Figure 4.10.D-E). In fact, the values of $R^{2}$ for the data under analysis covered the full range defined for this parameter, as it can be observed in Figure 4.11.

\begin{tabular}{|c|c|c|}
\hline & Mean \pm SD & Range \\
\hline$A(\mu V)$ & $26.38 \pm 12.89$ & $15.94-66.71$ \\
\hline$F_{A A}(H z)$ & $6.60 \pm 1.09$ & $4.59-8.98$ \\
\hline res & $0.05 \pm 0.03$ & $0.01-0.04$ \\
\hline$R^{2}$ & $0.56 \pm 0.12$ & $0.30-0.79$ \\
\hline
\end{tabular}

Table 4.5: Mean, standard deviation and range values of the parameters measured over the recorded Frank leads calculated from the mean values in all AF patients.

Figure 4.12 shows an example of recorded Frank leads versus Dower-derived orthogonal leads. In this example, derived orthogonal leads present a good 


\section{QRS complex}

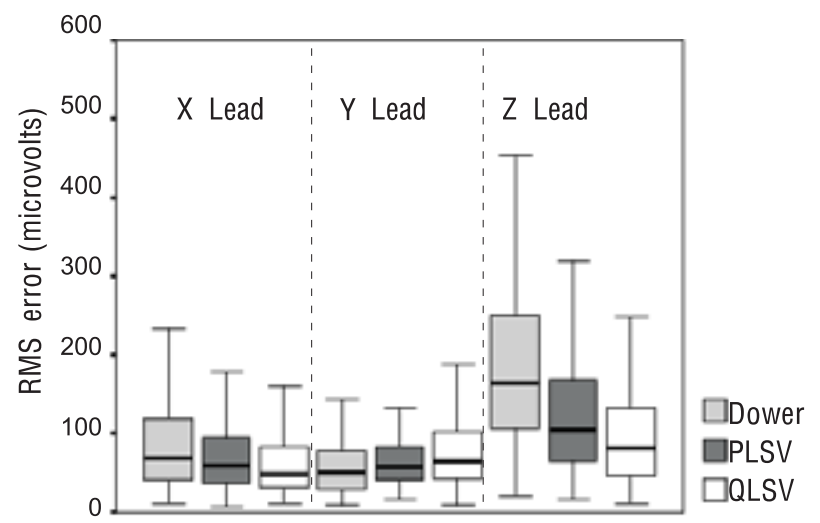

Figure 4.8: Root mean squared error of derived versus true measured QRS complexes in the three orthogonal leads X,Y,Z using Dower, PLSV and QLSV transforms

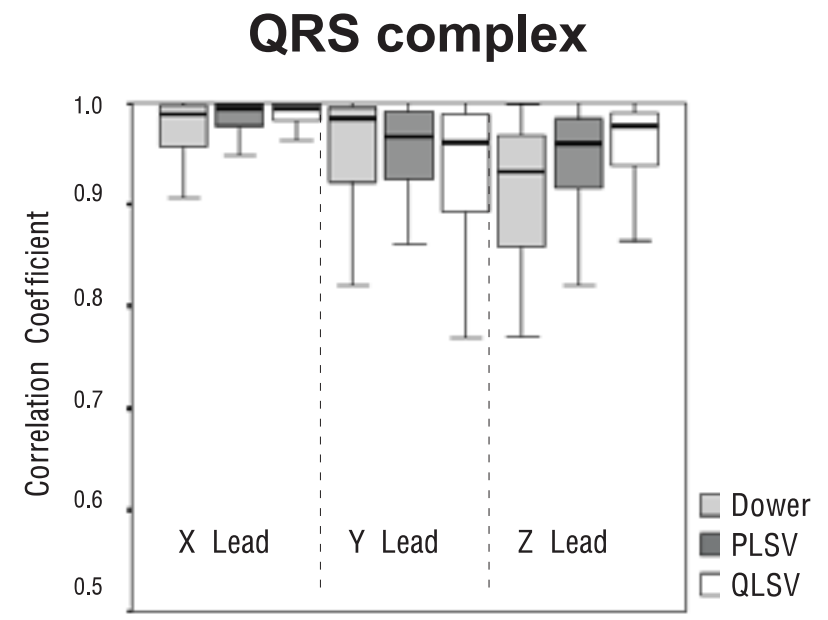

Figure 4.9: Correlation of derived vs. true measured QRS complexes. Correlation coefficients for Dower, PLSV and QLSV transforms 
A

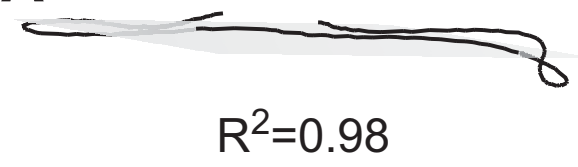

B

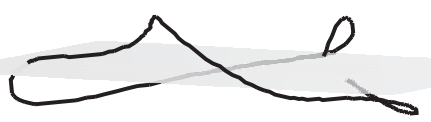

$\mathrm{R}^{2}=0.75$

C

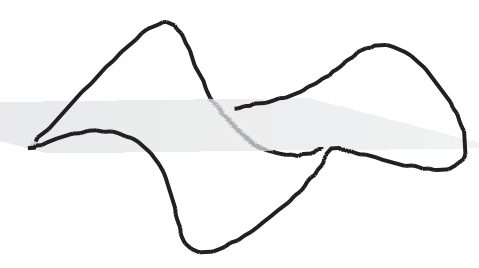

$\mathrm{R}^{2}=0.56$
D

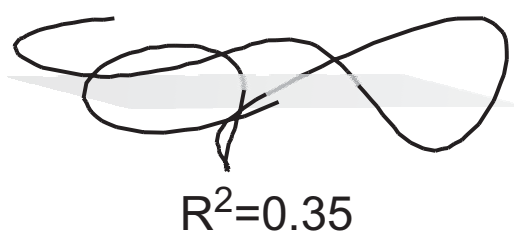

E

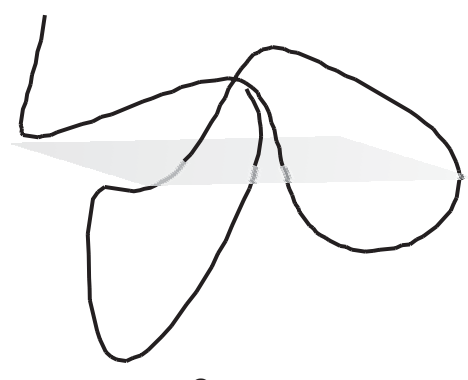

$\mathrm{R}^{2}=0.08$

Figure 4.10: Examples of different AF loops and their fit to a plane. Panels A-E show five different AF waves with different $R^{2}$ values together with the plane of best fit to each wave.

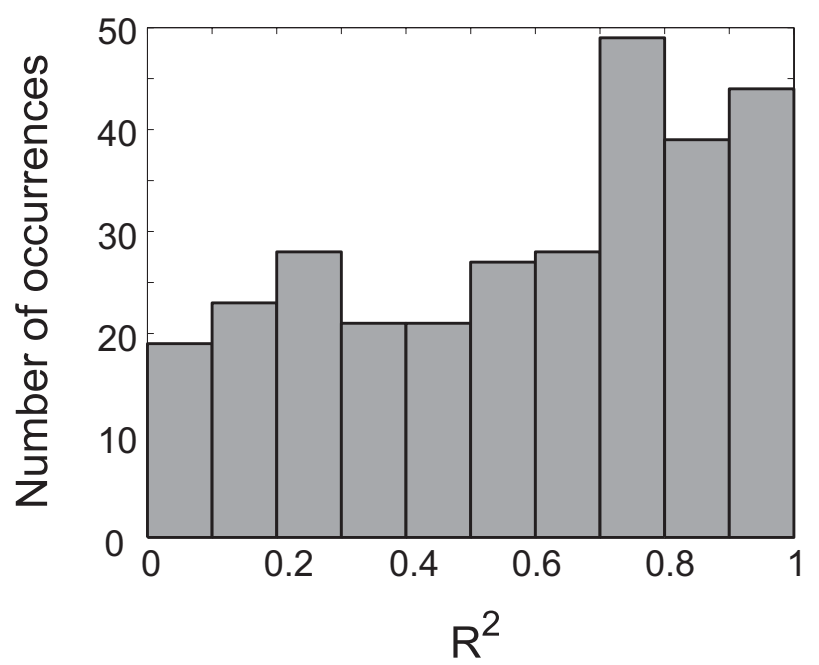

Figure 4.11: Histographic representation of the fit to the plane $\left(R^{2}\right)$ of $\mathrm{AF}$ waves. $R^{2}$ values were obtained for 299 waves (23 waves per patient) from the orthogonal Frank leads. 
correlation with Frank leads: $0.78(\mathrm{p}<0.01)$ for lead X, $0.98(\mathrm{p}<0.01)$ for lead $\mathrm{Y}$ and $0.95(\mathrm{p}<0.01)$ for lead Z. However, $\varepsilon_{R}$ is equal to 0.42 and $\varepsilon_{D}$ is equal to $27^{\circ}$.

A
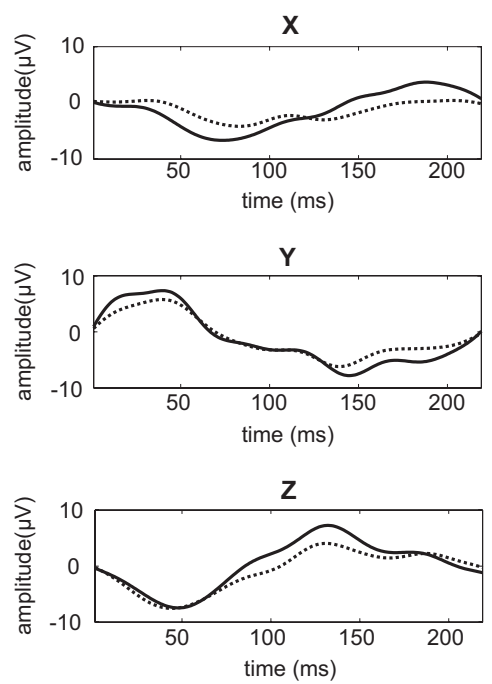

B

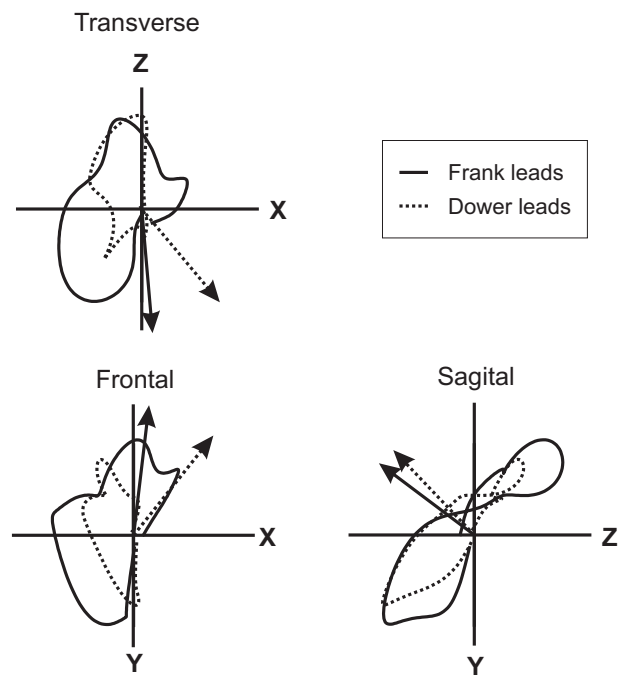

Figure 4.12: Franks versus derived orthogonal AF waves using Dower's inverse transform. Panel A shows leads X, Y, and Z corresponding to one AF segment. Solid line represents Frank leads. Dashed line represents derived leads. Panel $\mathrm{B}$ shows the projection of the vectorcardiogram into transverse, frontal and saggital planes and the direction given by the normal vector to the plane both for Frank leads and derived leads. Derivation of this particular segment was accomplished with a relative error equal to 0.43 and an error in the estimation of the orientation of $27.48^{\circ}$

Derivation of orthogonal waves with Dower's inverse transform was achieved with relative errors equal to $0.69 \pm 0.25$ (range: $[0.42-1.12]$ ) and errors in the estimation of orientation of $35.72^{\circ} \pm 9.54^{\circ}$ (range: $\left[19.11^{\circ}-56.55^{\circ}\right]$ ) (see Table 4.6). Derivation with PLSV transform achieved slightly lower errors than Dower's inverse transform: $\varepsilon_{R}=0.63 \pm 0.27(\mathrm{p}=\mathrm{ns})$ and $\varepsilon_{D}=32.89 \pm 9.12$ $(\mathrm{p}=\mathrm{ns})$.

Histographic representation of errors in reconstruction of all derived waves in our database by using Dower's inverse transform can be observed in Figure 4.13 .

Correlation among errors in derivation of AF waves and in the estimation of the orientation of AF loops by using Dower's inverse transform with parameters 


\begin{tabular}{|c|c|c|c|}
\cline { 3 - 4 } \multicolumn{2}{c|}{} & Mean \pm SD & Range \\
\hline \multirow{2}{*}{ Dower } & $\varepsilon_{R}$ & $0.69 \pm 0.25$ & $0.42-1.12$ \\
\cline { 2 - 4 } & $\varepsilon_{D}($ deg $)$ & $35.72 \pm 9.54$ & $19.11-56.55$ \\
\hline \multirow{2}{*}{ PLSV } & $\varepsilon_{R}$ & $0.63 \pm 0.27$ & $0.36-1.15$ \\
\cline { 2 - 4 } & $\varepsilon_{D}($ deg $)$ & $32.89 \pm 9.12$ & $19.66-48.23$ \\
\hline
\end{tabular}

Table 4.6: Mean, standard deviation and range values of the estimators of error in the derivation of orthogonal AF waves with Dower and PSLV transforms calculated from the mean values in all AF patients.

A

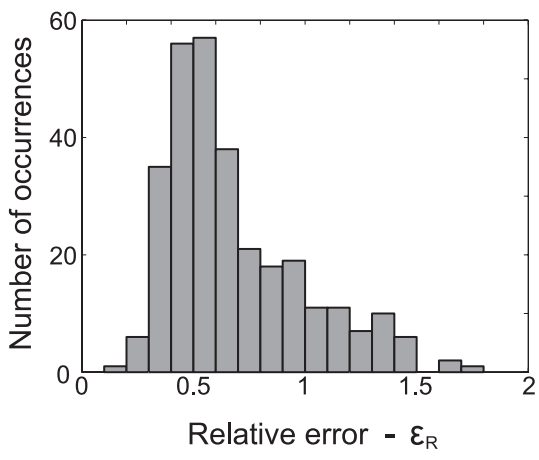

B

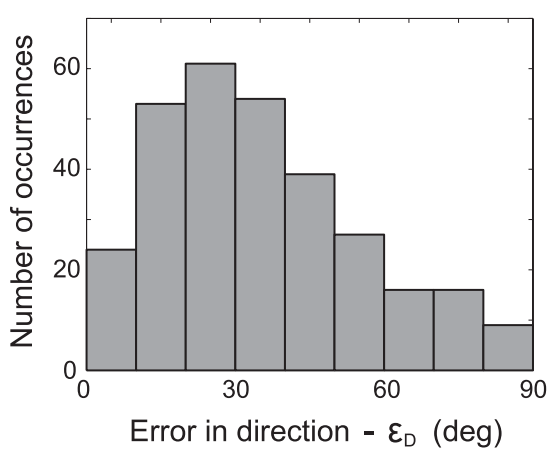

Figure 4.13: Histographic representation of errors in reconstruction of derived waves with Dower's inverse transform (23 waves per patient). Panel A shows the histographic representation of calculated relative errors of derived waves versus Frank leads. Panel B shows the histographic representation of the error in the estimated direction of the $\mathrm{AF}$ wave calculated from the planes of best fit of Frank waves versus derived waves. 
measured on Frank leads are presented in Table 4.7. No correlation was found among any of the parameters under study.

\begin{tabular}{|c|c|c|}
\hline & $\varepsilon_{R}$ & $\varepsilon_{D}$ \\
\hline$A(\mu V)$ & $-0.13^{*}$ & 0.00 \\
\hline$F_{A A}(H z)$ & -0.28 & -0.12 \\
\hline$r e s$ & 0.11 & 0.02 \\
\hline$R^{2}$ & $-0.18^{* *}$ & -0.09 \\
\hline
\end{tabular}

Table 4.7: Correlation among mean errors in reconstruction of AF waves and other measured parameters for 13 patients included in our database ${ }^{*}$ indicates a $\mathrm{p}$ value $<0.05,{ }^{* *}$ indicates a $\mathrm{p}$ value $<0.01$.

\subsection{Discussion}

\subsubsection{Main findings}

This study is the first to systematically evaluate the errors in derivation of orthogonal waves from the 12-lead ECG during atrial activity when using Dower's inverse transform. We first proposed a transform matrix optimized for the derivation of atrial waves which achieves a better performance on the derivation of $\mathrm{P}$ waves than Dower's inverse transform.

We tested this P-optimized matrix and Dower's inverse transform in the derivation of atrial fibrillatory waves. We found relative errors equal to $69 \%$ and errors in estimation of direction equal to $35.72^{\circ}$. Derivation using P-optimized matrix resulted non-statistically different errors. These errors were not related to AF amplitude, frequency, dipolarity of AF waves or their fit to a plane.

\subsubsection{Transform matrix optimized for the $\mathrm{P}$ wave}

Although it is clear that electrical activation occurs in different spatial locations relative to any lead set for the atria and the ventricles, it was unclear whether this difference in location has a significant effect on the derivation of orthogonal leads from the 12-lead ECG corresponding to atrial electrical activity.

First we evaluated the possibility of computing a transform matrix optimized for atrial activity. If inter-patient variability affects the optimal transform matrix to a greater extent than the difference in location of the atria and the ventricles then the advantage of using a different transform for the $\mathrm{P}$-wave might not be significant. Transform matrices were calculated for $\mathrm{P}$ waves in the study and test sets and they did not differ significantly. The reproducibility of the transform matrices confirmed the importance of the difference in location of the electrical activation over intra-patient variability. 
Also, we have shown that it is possible to construct a matrix based on LSV optimization for the $\mathrm{P}$ wave that globally improves on Dower's transform. However, this $\mathrm{P}$ wave transform offers a poorer performance on the derivation of Y lead than Dower's inverse transform. We have not investigated the origin of this inaccuracy in Y lead, however, we hypothesize that PLSV matrices computed for each individual may differ greatly for Y lead than for other leads and thus averaging of $\mathrm{Y}$ coefficients of different patients does not result in an optimized transform.

This P-optimized transform also performs better for the derivation of the QRS complex than Dower's transform for this database. This result can be partially attributed to the method used for the computation of the P-wave optimized matrix, namely LSV optimization. It could be questioned whether there is an additional influence of the time interval used for the optimization (P-wave or QRS complex) on the performance. LSV optimization for the QRS complex has been shown to offer better results for the derivation of orthogonal leads than Dower's transform $[81,110,136]$. However, derivation of the P-wave with a QRS-optimized transform has not been studied before, and it has not been assessed whether LSV optimization by itself performs better than Dower's inverse transform for the $\mathrm{P}$ wave with either waveform used for the optimization process.

In this study we also compared the derivation of the $\mathrm{P}$ wave by using a transform matrix optimized either for the P-wave or the QRS complex by using the LSV method. It was shown that the performance on the P-wave with the P-wave optimized transform was superior than the performance of the QRS complex optimized transform. Besides the benefit of LSV optimization, a Pwave optimized transform performs better on the $\mathrm{P}$ wave than a QRS-optimized transform.

Previous studies have derived optimized transform matrices for the QRS complex from larger datasets [77]. We tested the performance of this transform matrix on the $\mathrm{P}$ wave and the QRS complex for our database. Performance on the $\mathrm{P}$ wave was slightly poorer (root mean squared error on the $\mathrm{P}$ loop equal to $12.5 \pm 7.8 \mu \mathrm{VRMS}$ and correlation indexes for all leads also lower than those achieved with the PLSV transform) whereas on the QRS complex its performance was significantly higher (root mean squared error on the QRS complex equal to $70.0 \pm 38.3 \mu \mathrm{VRMS}$, whereas correlation values were higher in two out of the three orthogonal leads). This result reinforces our observation: a transform matrix optimized for the QRS complex offers a poorer performance on the $\mathrm{P}$ wave than a transform matrix optimized for the $\mathrm{P}$ wave.

\subsubsection{On the derivation of AF loops from the ECG}

Planes of best fit of AF loops have been used in the literature for measuring the orientation of AF loops [102, 106]. However, whether AF loops fit to a plane or not has not been evaluated before. From our results, only $38 \%$ of AF 
loops fitted to a plane if an $R^{2}$ value of 0.75 is considered to be sufficient. The meaning of any possible orientation parameter derived from a plane that does not fit the AF loop should be questioned.

Assuming that most AF loops do not fit a plane, other spatial parameters could be investigated in order to obtain information about the fibrillatory process in a given patient. But in case orthogonal leads are not available we should determine whether meaningful results can be obtained if these orthogonal leads are derived from standard leads before extracting any clinical conclusions. Dower's inverse transform has been widely used for the derivation of orthogonal waves from the 12-lead ECG despite the existence of more accurate methods [77]. Appropriateness of Dower's inverse transform for extraction of spatial properties of waves that correspond to atrial activity during sinus rhythm is even more doubtful. Although Carlson et al. [16] concluded that Dower's inverse transform is accurate for the derivation of the $\mathrm{P}$ wave by comparing individual leads, they did not compare any spatial indicators. Our findings on the $\mathrm{P}$ wave demonstrate that the accuracy of Dower's inverse transform on atrial $\mathrm{P}$ waves during sinus rhythm leaves room for improvement.

From our results, the use of Dower's inverse transform for the derivation of orthogonal leads that correspond to atrial fibrillatory signals leads to an average error of $68 \%$, and this error even exceeds the signal level in $16 \%$ AF waves. If orientation of the loop is calculated under these circumstances, a mean error of $35.86^{\circ}$ (ranging from $0^{\circ}$ to $90^{\circ}$ ) is not surprising. We found higher errors if orthogonal waves were derived by the transform proposed by Kors et al [77] (errors of $125 \%$ and $43.36^{\circ}$ ). Unfortunately, our $\mathrm{P}$ wave optimized matrix did not perform significantly better for the derivation of atrial fibrillatory loops. This observation precludes the difference in location of the atria with respect to the ventricles as the main reason for a poor derivation of atrial fibrillatory loops.

Aiming at finding characteristics of $\mathrm{AF}$ waves that could predict a low error in $\mathrm{AF}$ wave derivation, we investigated the correlation among amplitude, $\mathrm{AF}$ frequency, wave residuum and fit to the plane to relative errors and errors in the estimation of direction in derived orthogonal waves. We were expecting that high-amplitude waves or low-frequency AF would be easier to derive and thus derivation by Dower's inverse transform would be more successful. Also, we expected that less error in the estimation of direction by using planes of best fit would be found in AF loops that fitted a plane. But this was not the case. We did not find any correlation among any of the parameters measured and the errors in derivation. It may be the case that the chaotic electrical activity during AF prevents orthogonal leads to be easily determined from the 12-lead ECG, and especially under circumstances of low signal-to-noise ratios.

All transformations tested rely on the assumption that the independent leads record the same electrical event. While this is may be true for ventricular 
activation and maybe also for AFL this can be questioned for $\mathrm{AF}$ waves. In other words, lead $V_{1}$ is related to right atrial activity while leads I and $V_{5}$ may be more influenced by left atrial activity. Apparently, different atrial areas may be activated independently of each other.

\subsubsection{Limitations of the study}

In this study only one database was used for both the calculation of the $\mathrm{P}$ wave optimized transform matrix and the evaluation of its performance. Although patients were randomly subdivided into two groups (study and test groups), it is possible that the performance of the transforms we obtained would not be optimal when using a different database due to systematic errors in the placement of the electrodes. Further, the template matching used was not based on $\mathrm{P}$ wave averaging for the reasons outlined in the Methods section. If anything, this would have degenerated the performance of the $\mathrm{P}$ wave based transform.

A main limitation of our study is the small number of AF patients included. This may limit the generalization of our results relative to the derivation of $\mathrm{AF}$ loops, which may be valid only for our population under study.

\subsection{Conclusions}

We have shown that Dower's inverse transform is not the best method for deriving orthogonal leads from the 12-lead ECG for atrial waves.

A transform obtained with LSV optimization by using the P-wave offers better performance during sinus rhythm. Also, raising the question of whether it was the optimization for the P-wave or only the specific database that resulted in improvement over Dower's transform, we have shown that, for this same database, the $\mathrm{P}$-wave optimized transform performed better for the $\mathrm{P}$-wave while the QRS complex-optimized transform performed better for the QRS complex.

However, both Dower's inverse matrix and a P-wave optimized matrix offer a poor performance on the derivation of AF loops. Although derived waves may present some resemblance to orthogonal waves, the orientation of AF loops may be completely different and thus spatial parameters obtained after derivation may differ to those obtained from recorded orthogonal leads.

We conclude that the difference in the spatial position of electrical activation relative to the recording lead set seems to have a significant influence on the optimal P-wave derivation method. As a consequence, derivation of orthogonal leads for both the $\mathrm{P}$-wave and the QRS complex can be improved by using separate transform matrices. However, this difference in the spatial position of the electrical activation is not the main problem in the derivation of AF loops. Instead, the chaotic activation during AF that cannot be assumed as a single dipole may prevent the derivation of AF loops from the standard ECG. 
If the atrial vectorcardiogram in sinus rhythm is to be studied from the 12lead ECG, the use of a specific transform for the P-wave is more accurate than other transform matrices, while if the atrial vectorcardiogram in AF is to be studied, it cannot be derived from the 12-lead ECG. 


\section{Chapter 5}

\section{Optimization of ECG leads ${ }^{1}$}

\subsection{Introduction}

5.2 Evaluation of lead selection methods

\author{
5.3 Selection of ECG \\ leads for AF \\ 5.4 Conclusions
}

In the previous chapter it has been demonstrated that spatial characteristics of AF wavefronts cannot be derived from the standard ECG. This finding can be attributed to the lack of dipolarity of AF signals. More accurate spatial parameters of AF waves can be derived from the distribution of potentials during $\mathrm{AF}$ on the entire surface of the thorax. In this chapter we aim at determining the number and location of electrodes needed for an accurate reconstruction of body surface potentials during AF.

\footnotetext{
${ }^{1}$ Chapter based on the manuscripts:
}

- M. S. Guillem, F. Castells, A. M. Climent, V. Bodi, F. J. Chorro and J. Millet. Evaluation of lead selection methods for optimal reconstruction of body surface potentials. Journal of Electrocardiology 41: 26-34, 2008.

- M. S. Guillem, A. Bollmann, A. M. Climent, D. Husser, J. Millet and F. Castells. How many leads are necessary for a reliable reconstruction of surface potentials during atrial fibrillation?. IEEE Transactions on Information Technology in Biomedicine (Under revision). 


\subsection{Introduction}

The 12-lead electrocardiogram (ECG) is the most extended non-invasive technique in cardiology, due to its capability to assess reliably cardiac disorders in a simple and inexpensive manner. Despite the relatively high amount of electrodes required to obtain the 12-lead ECG (9 electrodes), the total amount of information provided by all them has been questioned [84, 127]. Precordial leads $V_{1}$ to $V_{6}$ are positioned close to each other on the body surface, and some of them are quite far from the atria. As a result, the standard leads are highly correlated and they exhibit a high amount of mutual information. In addition, the precordial leads were originally positioned for capturing the ventricular electrical activity, so the atrial activity may not be accurately reflected at these sites.

Body surface potential mapping (BSPM) studies have shown that, in some cases, there is diagnostic information that may be inadequately sampled by the standard 12-lead ECG and thus the diagnosis of some cardiac diseases would benefit from the use of a higher number of electrodes. Benefit of the analysis of body surface maps is not only restricted to ventricular diseases and it has been applied in the characterization of atrial activity in sinus rhythm and the localization of ectopic atrial activation $[118,117,116]$.

Practical use of BSPM systems is limited by the tedious of the recording procedure and for this reason, the benefit of employing a high number of leads has been questioned. Previous studies concerning the selection of leads from BSPM systems agree in considering that the total information content in multi-lead recordings is not as high as the number of electrodes employed for recording electrocardiographic signals, being 30 to 32 electrodes sufficient for recording ventricular activity in most heart conditions [85, 62, 78]. These results have motivated the proposal of limited lead systems including non-standard leads with the aim of improving the diagnostic information of the ECG. As an example new electrode positions outside the 12-lead ECG have been proposed for improving the diagnosis of Brugada's syndrome $[60,128,111]$.

A feasible approach for determining optimum electrode locations for specific pathological conditions makes use of BSPM recordings for determining the leads with higher information content out of the total number of leads employed and different methods have been proposed at this aim. These previous studies about the information content of multiple electrocardiographic leads have mainly been focused in optimizing the global information content of the whole ECG. In this case the activity captured during the ventricular depolarization (i.e. the QRS complexes) predominates over any other cardiac activity. The nature of this electrical activity are much different from the atrial fibrillatory signals, for several reasons: (1) they have their origin in different parts of the heart, (2) the fibrillatory signals are consequence of a chaotic activation in contrast to the organized activation of a 
non-fibrillating ventricle and (3) the fibrillatory signals exhibit a lower signal-to-noise ratio. Taking into account these considerations, it can be inferred that the model applicable to the whole ECG may not longer match the atrial fibrillatory waves. The issue of information content of lone atrial fibrillatory signals has not been addressed before. Previous studies have focused on proposing new reduced lead systems for improving the diagnosis and therapy for atrial fibrillation [66, 67]. Furthermore, new electrode positions have been also investigated for minimizing errors in the reconstructed vectorcardiogram in atrial fibrillation [131].

The objective of this study is to evaluate the suitability of the standard electrocardiogram in AF and to compare its performance to other limited lead systems. First we evaluated two different lead selection algorithms [88, 67] for BSPM recordings. After evaluation of lead selection algorithms in non-fibrillating rhythms, we applied the method that offers a better performance in the determination of optimum lead sets for the study of AF. We made use of 64-lead BSPM recordings of patients during atrial fibrillation and we experimentally determined how many leads are necessary for accurately reconstructing the potentials on their whole body surface in terms of the RMS error of reconstructed leads. The desired accuracy is limited by the noise level, and thus a perfect reconstruction cannot be achieved. A more reasonable objective is to achieve an accuracy in the reconstruction of atrial fibrillatory waves as that achieved in the reconstruction of QRS complexes or $\mathrm{P}$ waves by using the standard 12-lead ECG. With this aim, we performed the same experiments over the QRS complex and $\mathrm{P}$ wave of healthy subjects in order to have a reference in the accuracy achievable for those waves in limited lead systems. Besides from measuring errors in reconstruction of non-selected leads, we evaluated the similarity of spectral features of reconstructed leads to those of the recorded ECG signals as an independent diagnostic parameter that is frequently used in the study of atrial fibrillation.

Also, new lead positionings will be evaluated aimed at improving the information content of atrial fibrillatory signals recorded by limited lead systems. A non-standard 12-lead ECG system will be proposed as a practical solution for improving the diagnosis of atrial fibrillation. In order to keep some coherence with the standard ECG, this lead system will include some of the standard leads and will allow a repositioning of four precordial leads. The performance of such a lead system will be evaluated. 


\subsection{Evaluation of lead selection methods}

\subsubsection{Materials and Methods}

\section{Study Population}

One minute BSPM recordings were obtained from patients with different diagnosis admitted in HCUV and healthy subjects with electrode configuration 1 (see section 3). In order to have an homogeneous database, we randomly selected 18 patients from each of the following groups: Control, Myocardial Infarction (MI), Bundle Branch Block (BBB) and Ventricular Hypertrophy $(\mathrm{VH})$, having a whole database consisting of 72 patients.

\section{Signal processing}

Segments of ten seconds of every patient were considered in our study. Signals were band-pass filtered (high pass filter: cutoff frequency $0.5 \mathrm{~Hz}$ and low pass filter: cutoff frequency $70 \mathrm{~Hz}$ ) in order to avoid noise to corrupt the results. QRS peaks were detected by using a modified version of Tompkins algorithm. Offset of the electrodes was reduced by subtracting the mean value in the PQ interval, where the PQ interval was estimated as the region between $[0.6 \cdot \mathrm{RR}, 0.7 \cdot \mathrm{RR}]$ after each QRS peak, where RR denotes the length of the RR interval being considered. Signal quality in all patients and all leads was visually inspected and leads presenting an unacceptable level of noise were discarded and interpolated from their neighboring leads.

QRS segments were defined by using a window of a fixed length of 100 ms around the QRS peak in $V_{1}$. P segments were defined by using a window of a fixed length of $150 \mathrm{~ms}$ and starting $200 \mathrm{~ms}$ before the QRS peak in $V_{1}$. Segment definition was visually inspected for all patients and all leads and segment duration or positioning was manually edited when needed.

\section{Organization of the database}

In order to proceed with the lead selection, a segment of 10 seconds of the potential signals of all patients within a group were concatenated, so that a new potential matrix (e.g. $\mathbf{X}_{\mathrm{BBB}}$ ) with a duration of 180 seconds was created for each of the groups. Furthermore, an additional matrix $\mathbf{X}_{\text {global }}$ containing the potential signals of all patients in the database was generated to represent all groups. Finally, group potential signals containing only the QRS-complexes (e.g. $\mathbf{X}_{\mathrm{QRS}_{\mathrm{BBB}}}$ ), as well as an additional matrix containing the $\mathrm{P}$-waves and another containing only QRS-complexes of all patients were also created (e.g. $\mathbf{X}_{\mathrm{P}_{\text {global }}}$ and $\mathbf{X}_{\mathrm{QRS}_{\text {global }}}$ ).

Since the optimal lead selection may be different for each patient (or group of patients), each of the groups has been divided into a study and test subgroups, both sets containing the same number of patients. Obviously, the best results 
will be obtained when the lead selection and performance analysis are applied over the same database. However, under an actual situation where the ECG of a patient must be analyzed with a limited number of leads, the optimal lead position for that patient is a priori unknown. In order to compare the algorithms performance in an ideal and a real situation, the lead selection will be carried out over the study set, whereas the performance analysis will be evaluated over both the study and the test sets.

\section{Lead selection}

Two methods for lead selection described in the literature were implemented. Obviously, the best lead selection can be always determined by evaluating all possible combinations and choosing the one that best matches the reconstruction of the remaining ECGs. However, this approach would be not affordable from a computational point of view, since it would require over $10^{19}$ evaluations for a 64-lead system. In the case of a higher number of leads, the total amount of evaluations increases exponentially (e.g. over $10^{38}$ for a 128-lead system). With the aim of reducing the computational complexity, two suboptimal methods already proposed in previous studies and based on different criteria have been implemented: method 1 based on the analysis of the spatial correlation among recording sites [88] (see section 2.4.1) and method 2 based on the analysis of the number of independent signals in multilead recordings [67] (see section 2.4.1).

The results obtained with these methods will be also compared to the results that would be obtained using a selection of the conventional 12-lead ECG. Unipolar leads that correspond to the 12-lead ECG were ordered with the objective of comparing the information of the 12-lead ECG with the information contained in the lead sets derived from the other selection methods. The sequence of leads: right arm, left leg, $V_{1}, V_{3}, V_{5}, V_{2}, V_{6}, V_{4}$ was chosen in an attempt of optimizing the information of every lead subset as it was done in the other methods of selection.

\section{Evaluation of lead selections}

In order to evaluate the lead sets that have been obtained by the different methods detailed above, we derive non-selected leads and compare true recorded versus derived leads. The best lead set will be defined as the lead set that derives the rest of the leads more accurately.

Having the leads ordered as they result from each lead selection algorithm we calculated derived leads from an increasing number of selected leads as a linear combination of them. The linear combination $\mathbf{T}$ that optimizes the derivation is calculated by Least Squares Optimization:

$$
\hat{\mathbf{X}}_{\left\{i_{1}, \ldots, i_{N}\right\}}=\mathbf{T} \cdot \mathbf{X}_{\left\{j_{1}, \ldots, j_{M}\right\}}
$$


Where $\mathbf{X}\{\cdot\}$ is a matrix containing the rows of $\mathbf{X}$ corresponding to the indexes in $\{\cdot\},\left\{j_{1}, \ldots, j_{M}\right\}$ are the indexes of the selected leads, $\hat{\mathbf{X}}$ is a matrix of estimated non-selected leads, $\left\{i_{1}, \ldots, i_{N}\right\}$ are the indexes of nonselected leads and $\mathbf{T}$ is the transform that optimizes the estimation. $\mathbf{T}$ matrix is computed by using both selected leads and non-selected leads of the study dataset set as follows:

$$
\mathbf{T}=\mathbf{X}_{\left\{i_{1}, \ldots, i_{N}\right\}} \cdot \mathbf{X}_{\left\{j_{1}, \ldots, j_{M}\right\}}^{\dagger}
$$

Where $X^{\dagger}$ is the Moore-Penrose pseudo-inverse of matrix $X$. Derived leads were compared with true recorded leads by calculating the mean error and correlation values. Mean error was defined as:

$$
\varepsilon_{M}=\frac{\sum_{l=1}^{L} \sqrt{\frac{\sum_{n=1}^{S}\left(\mathbf{X}_{(l, n)}-\hat{\mathbf{X}}_{(l, n)}\right)^{2}}{S}}}{L}
$$

where $M$ stands for the number of leads that constitute the lead set under evaluation, ranging from 1 to $L$ and $S$ stands for the number of samples of the ECG matrix under evaluation. Mean correlation was defined as:

$$
\rho_{M}=\frac{1}{L} \sum_{l=1}^{L} \rho_{x_{l}, \hat{x}_{l}}
$$

where $\rho_{x_{l}, \hat{x}_{l}}$ stands for the correlation coefficient between lead $x_{l}$ and derived lead $\hat{x}_{l}$ :

$$
\rho_{x_{l}, \hat{x}_{l}}=\frac{S \sum_{n} \hat{x}_{l}(n) \cdot x_{l}(n)-\sum_{n} x_{l}(n) \cdot \sum_{n} \hat{x}_{l}(n)}{\sqrt{S \sum_{n} x_{l}(n)^{2}-\left(\sum_{n} x_{l}(n)\right)^{2}} \cdot \sqrt{S \sum_{n} \hat{x}_{l}(n)^{2}-\left(\sum_{n} \hat{x}_{l}(n)\right)^{2}}}
$$

In order to evaluate the performance of the algorithms, two experiments are performed. The first experiment refers to an ideal situation where the lead selection, the transformation matrix $\mathbf{T}$ and the parameters $\varepsilon$ and $\rho$ are applied over the same ECG segment, i.e. the test dataset. This would reflect the maximum performance that can be achieved by each algorithm, since the optimum lead system and transformation matrix are available for the ECG segments under evaluation. The second experiment refers to a real situation where neither the optimum lead system nor the optimum transformation matrix for the ECG segment under test are available, i.e. the test dataset. In this case, the lead system is derived from the corresponding patient group in the study dataset, whereas the transformation matrix is obtained from the entire ECG segments in the study dataset and will be considered the same independently of the patient group under evaluation. 


\subsubsection{Results}

\section{Leads Selected}

The leads selected by the implemented methods resulted in a different selection of leads. These selection methods were applied for the definition of optimized lead sets for the complete ECG signals, the QRS segments and also for only the $\mathrm{P}$-waves. The order in which the leads are selected differs from method 1 to method 2, and it depends also on the patient group under study, as it can be observed in figure 5.1, where the first eight leads to be selected by each of the methods are shown.

A. Xglobal Method 1

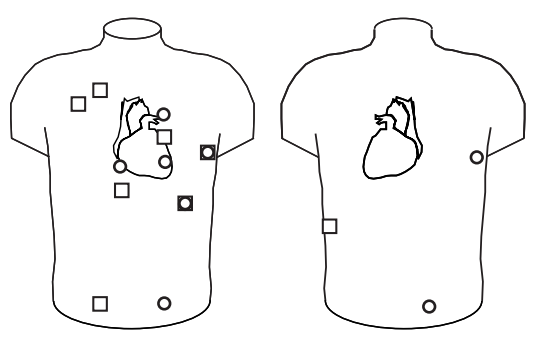

- Leads selected for the test set $\square$ Leads selected for the study set

\section{С. Хввв Method 1}

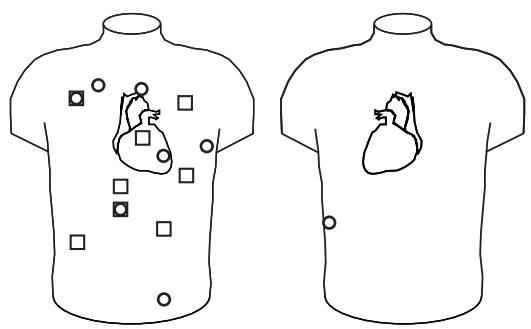

- Leads selected for the test set Leads selected for the study set
B. Xglobal Method 2

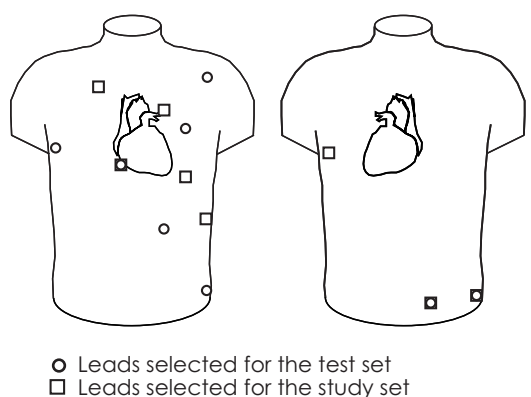

D. XP Method 1

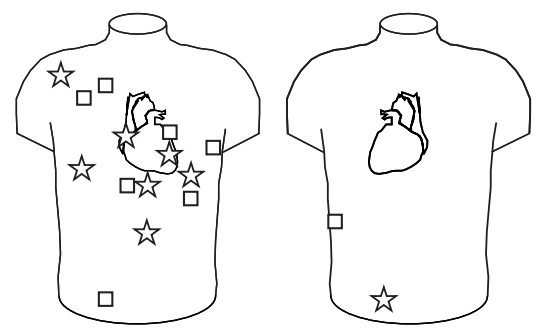

th Leads selected for the study set and the P wave $\square$ Leads selected for the study set and the ECG global

Figure 5.1: Leads selected for different datasets and selection methods. Panels A and $\mathrm{B}$ show the first eight leads selected by method 1 and method 2 respectively for the study and test datasets using the whole ECG of all patients. Panel C shows the first eight leads selected by method 1 for both the study and test sets of the BBB group. Panel D shows the first eight leads selected by method 1 using the $\mathrm{P}$ wave of all patients included in the study set. Leads selected by the same method and group of patients for the whole ECG are also displayed.

Figure 5.1-A shows the first eight leads selected by method 1 for both the 
study and test sets. Although some lead positions are selected in both groups, such as the precordial area, there are leads which differ from one group to another: two leads are selected on the upper right side of the torso for the study set while no lead was selected in this region for the test set and the distribution of leads on the back is completely different. Leads selected for the same groups of patients with method 2 can be observed in 5.1-B. Lead positions are similar to those observed in 5.1-A and there is little difference among leads selected for the study and test sets.

Figure 5.1-C shows the first eight leads selected by method 1 for the BBB group, also divided into study and test sets. Leads are placed following a similar pattern in both groups although no lead was selected on the back for the study set, while one lead was selected on the leftmost part of the back for the test set.

Leads selected for the P-wave and method 1 are presented in 5.1-D. Only small differences in lead placement can be observed from those leads selected by the same method on the whole ECG $\mathbf{X}_{\text {global }}$.

\section{Evaluation of lead selections}

The performance of each of the lead selection methods was tested according to the evaluation criteria previously defined. Evaluation was performed for each of the groups separately and also for a database containing patients from all groups in the study. As aforementioned, two different scenarios are investigated: (1) the selected leads, the transformation matrix and the evaluation of the reconstructed potentials are carried out over the test dataset (ideal case) and (2) the selected leads and the transformation matrix are obtained from the study dataset, whereas the evaluation of the reconstructed potentials is carried out over the test dataset (real case).

Figure 5.2 shows the results obtained considering jointly all patient groups (control, MI, BBB and VH) divided into study and test sets. It represents the error in reconstruction of non-selected leads for an increasing number of selected leads. For 3 leads in an ideal case the reconstruction error was $55.26 \mu \mathrm{V}$ for method 1, $68.03 \mu \mathrm{V}$ for method 2 and $56.79 \mu \mathrm{V}$ for a subset of the 12-lead ECG, which increased up to $72.03 \mu \mathrm{V}, 88.02 \mu \mathrm{V}$ and $66.58 \mu \mathrm{V}$, for method 1 , method 2 and a subset of the 12-lead ECG, respectively under a real scenario (see table 5.1). With 8 leads in an ideal case, the errors in reconstruction are: $36.70 \mu \mathrm{V}$ for method $1,44.48 \mu \mathrm{V}$ for method 2 and $39.58 \mu \mathrm{V}$ for the 12-lead ECG, which increased up to $42.86 \mu \mathrm{V}, 52.06 \mu \mathrm{V}$ and $49.69 \mu \mathrm{V}$, for method 1 , method 2 and the 12-lead ECG when the performance is evaluated in a real case (see table 5.2).

As electrically inactive segments may influence the averaged error, we computed the errors for the QRS complex separately. In fact, the reconstruction errors over the QRS segment considering 8-lead sets and a real case increased up to $105.84 \mu \mathrm{V}, 106.81 \mu \mathrm{V}$ and $130.06 \mu \mathrm{V}$ for method 1, method 2 and the 12-lead ECG (see tables 5.1 and 5.2 and compare the results of 


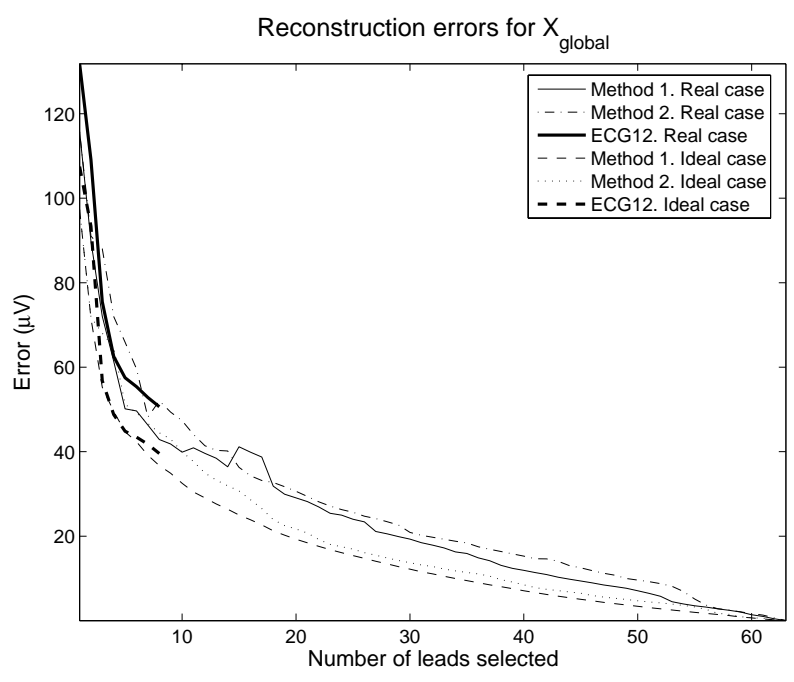

Figure 5.2: Error for limited lead sets on the whole ECG. Comparison of real and ideal case with both lead selection methods

$\mathbf{X}_{\text {global }}$ and $\mathbf{X}_{\mathrm{QRS}_{\text {global }}}$ for more detailed information).

In addition to reconstruction errors, correlation values are shown in Figure 5.3. Correlation values computed over $\mathbf{X}_{\text {global }}$ for 3 leads in an ideal case were: 0.874 for method 1, 0.777 for method 2 and 0.878 for a subset of the 12-lead ECG, which decreased to $0.863,0.772$ and 0.856 for method 1 , method 2 and a subset of the 12-lead ECG, respectively when evaluated in a real case (see table 5.3). With 8 leads in an ideal case, the correlation values are: 0.931 for method 1, 0.918 for method 2 and 0.918 for the 12-lead ECG, which decreased to $0.946,0.923$ and 0.895 , for method 1, method 2 and the 12-lead ECG under a real situation (see table 5.2).

Error and correlation values for limited lead sets were calculated for the complete set of patients and for each group separately, as it was expected that the presence of different pathologies resulted in a different performance of each lead set. Although for most groups, the performance was similar to the global performance, some differences could be observed in the BBB group, which presented lower performance than the rest of the groups. As an example, for method 1 considering 8 leads and a real case, the performance obtained for the BBB group decreased in comparison to the global ECG, with a reconstruction error of $93.56 \mu \mathrm{V}$ and a correlation of 0.814 (see tables 5.1-5.4 for additional information regarding the results obtained with this patient group). Figure 5.4 shows the errors in reconstruction of the whole ECG of limited lead sets with an increasing number of selected leads for the BBB group. As can be observed in this figure, errors in the ideal case monotonically decrease as the number of 


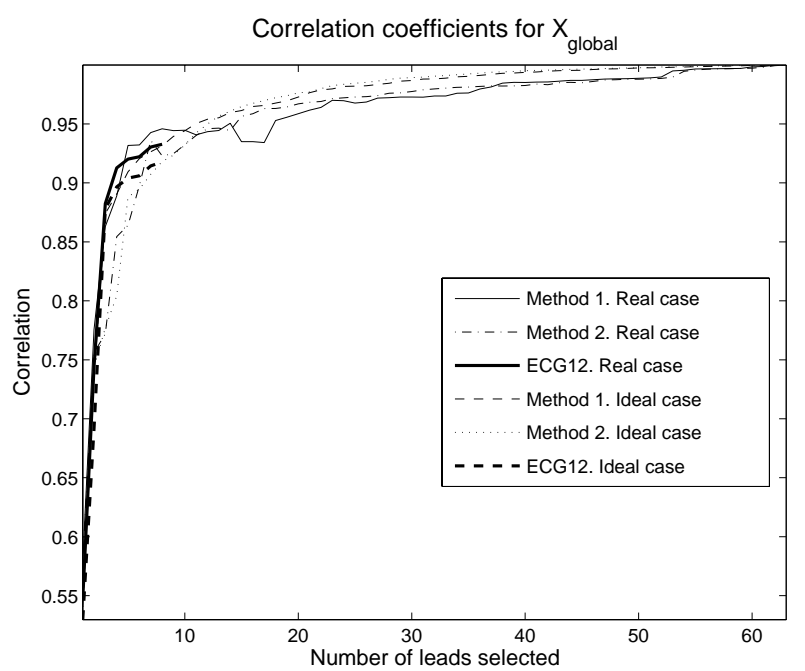

Figure 5.3: Correlation coefficients for limited lead sets on the whole ECG. Comparison of real and ideal case with both lead selection methods

\begin{tabular}{lcccccc} 
& \multicolumn{2}{c}{ Method 1 } & \multicolumn{2}{c}{ Method 2 } & \multicolumn{2}{c}{ 12-lead ECG } \\
ECG segment & Real & Ideal & Real & Ideal & Real & Ideal \\
\hline $\mathbf{X}_{\text {global }}$ & 72.03 & 55.26 & 88.02 & 68.03 & 66.58 & 56.79 \\
$\mathbf{X}_{\mathrm{QRS}_{\text {global }}}$ & 180.69 & 149.09 & 219.88 & 165.58 & 183.49 & 163.28 \\
$\mathbf{X}_{\mathrm{BBB}}$ & 122.67 & 82.76 & 262.73 & 107.93 & 109.34 & 102.14 \\
$\mathbf{X}_{\mathrm{QRS}_{\mathrm{BBB}}}$ & 262.29 & 181.73 & 259.22 & 188.29 & 241.43 & 222.78 \\
$\mathbf{X}_{\mathrm{P}_{\text {global }}}^{*}$ & 20.12 & 17.98 & 22.95 & 19.99 & 24.35 & 19.56 \\
$\mathbf{X}_{\mathrm{P}_{\text {global }}^{* *}}^{*}$ & 19.90 & 18.44 & 26.98 & 20.65 & 24.35 & 19.56 \\
\hline${ }^{*}$ Lead selection is derived from $\mathbf{X}_{\mathrm{P}_{\text {global }}}$ & & & \\
${ }^{* *}$ Lead selection is derived from $\mathbf{X}_{\text {global }}$ & & & &
\end{tabular}

Table 5.1: Values of $\varepsilon_{3}$ for 3-lead sets obtained from different lead selection methods and ECG segments. All values are expressed in $\mu V$. 


\begin{tabular}{lcccccc} 
& \multicolumn{2}{c}{ Method 1 } & \multicolumn{2}{c}{ Method 2 } & \multicolumn{2}{c}{ 12-lead ECG } \\
ECG segment & Real & Ideal & Real & Ideal & Real & Ideal \\
\hline $\mathbf{X}_{\text {global }}$ & 42.86 & 36.70 & 52.06 & 44.48 & 49.69 & 39.58 \\
$\mathbf{X}_{\mathrm{QRS}}{ }_{\text {global }}$ & 105.84 & 76.29 & 106.81 & 94.76 & 130.06 & 99.57 \\
$\mathbf{X}_{\mathrm{BBB}}$ & 93.56 & 43.73 & 110.03 & 49.15 & 71.86 & 59.34 \\
$\mathbf{X}_{\mathrm{QRS}_{\mathrm{BBB}}}$ & 172.29 & 93.48 & 160.02 & 102.07 & 144.80 & 109.57 \\
$\mathbf{X}_{\mathrm{P}_{\text {global }}}^{*}$ & 15.16 & 13.11 & 17.58 & 15.23 & 17.51 & 14.74 \\
$\mathbf{X}_{\mathrm{P}_{\text {global }}^{* *}}^{* *}$ & 15.47 & 13.77 & 18.25 & 14.54 & 17.51 & 14.74 \\
\hline${ }^{*}$ Lead selection is derived from $\mathbf{X}_{\mathrm{P}_{\text {global }}}$ & & & \\
${ }^{* *}$ Lead selection is derived from $\mathbf{X}_{\text {global }}$ & & &
\end{tabular}

Table 5.2: Values of $\varepsilon_{8}$ for 8-lead sets obtained from different lead selection methods and ECG segments. All values are expressed in $\mu V$.

\begin{tabular}{lcccccc} 
& \multicolumn{2}{c}{ Method 1 } & \multicolumn{2}{c}{ Method 2 } & \multicolumn{2}{c}{ 12-lead ECG } \\
ECG segment & Real & Ideal & Real & Ideal & Real & Ideal \\
\hline $\mathbf{X}_{\text {global }}$ & 0.863 & 0.874 & 0.772 & 0.777 & 0.856 & 0.878 \\
$\mathbf{X}_{\mathrm{QRS}_{\text {global }}}$ & 0.837 & 0.906 & 0.741 & 0.860 & 0.871 & 0.891 \\
$\mathbf{X}_{\mathrm{BBB}}$ & 0.814 & 0.881 & 0.621 & 0.820 & 0.876 & 0.882 \\
$\mathbf{X}_{\mathrm{QRS}_{\mathrm{BBB}}}$ & 0.841 & 0.893 & 0.848 & 0.891 & 0.876 & 0.885 \\
$\mathbf{X}_{\mathrm{P}_{\text {global }}^{*}}^{*}$ & 0.852 & 0.875 & 0.802 & 0.842 & 0.824 & 0.863 \\
$\mathbf{X}_{\mathrm{P}_{\text {global }}^{*}}$ & 0.847 & 0.865 & 0.715 & 0.833 & 0.824 & 0.863 \\
\hline *ead selection is derived from $\mathbf{X}_{\mathrm{P}_{\text {global }}}$ & & & \\
${ }^{* *}$ Lead selection is derived from $\mathbf{X}_{\text {global }}$ & & &
\end{tabular}

Table 5.3: Values of $\rho_{3}$ for 3-lead sets obtained from different lead selection methods and ECG segments. All values are expressed in $\mu V$.

\begin{tabular}{lcccccc} 
& \multicolumn{2}{c}{ Method 1 } & \multicolumn{2}{c}{ Method 2 } & 12-lead ECG \\
ECG segment & Ideal & Real & Ideal & Real & Ideal & Real \\
\hline $\mathbf{X}_{\text {global }}$ & 0.946 & 0.931 & 0.923 & 0.918 & 0.895 & 0.918 \\
$\mathbf{X}_{\mathrm{QRS}}$ global & 0.933 & 0.966 & 0.931 & 0.940 & 0.923 & 0.944 \\
$\mathbf{X}_{\mathrm{BBB}}$ & 0.875 & 0.961 & 0.842 & 0.952 & 0.931 & 0.945 \\
$\mathbf{X}_{\mathrm{QRS}}$ & 0.912 & 0.968 & 0.894 & 0.955 & 0.942 & 0.961 \\
$\mathbf{X}_{\mathrm{BBB}}^{*}$ & 0.893 & 0.921 & 0.897 & 0.915 & 0.872 & 0.902 \\
$\mathbf{X}_{\mathrm{P}_{\text {global }}^{* *}}^{* *}$ & 0.891 & 0.916 & 0.881 & 0.919 & 0.872 & 0.902 \\
\hline${ }^{*}$ Lead selection is derived from $\mathbf{X}_{\mathrm{P}_{\text {global }}}$ & & & \\
${ }^{* *}$ Lead selection is derived from $\mathbf{X}_{\text {global }}$ & & &
\end{tabular}

Table 5.4: Values of $\rho_{8}$ for 8-lead sets obtained from different lead selection methods and ECG segments. All values are expressed in $\mu V$. 
leads increases, whereas this behavior cannot be always reproduced in the real case (notice that it may occur that by adding a new lead the overall performance decreases).

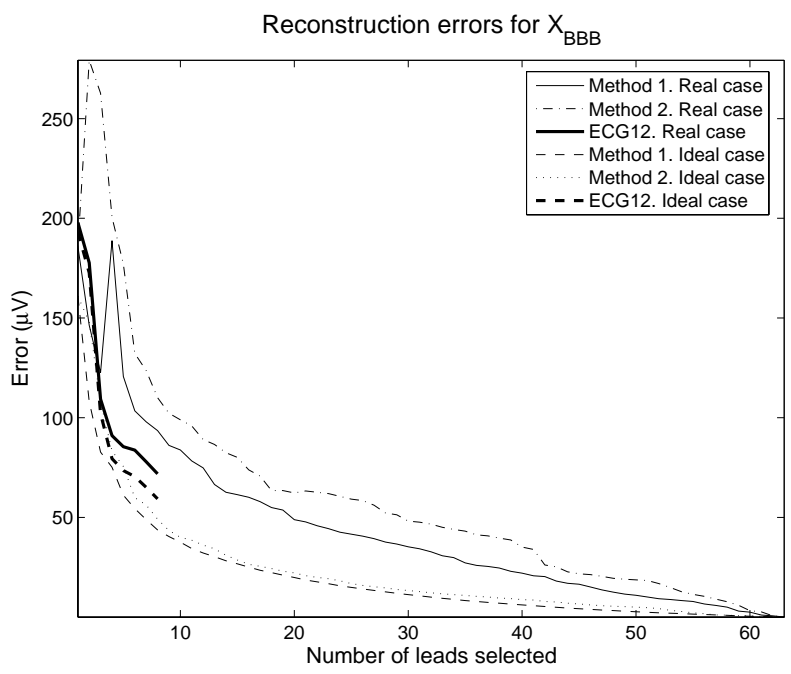

Figure 5.4: Error for limited lead sets on the whole ECG for the BBB group. Comparison of real and ideal case with both lead selection methods

Errors calculated for the BBB group and within the QRS complex can be observed in figure 5.5. These errors were higher than the errors for the QRS segment in the database containing all groups and also higher than the errors computed over the whole segment of the BBB group. Considering 8 leads in a real case, the errors obtained with method one were $172.29 \mu \mathrm{V}$ and the correlation was 0.912 (see tables 5.1-5.4 for more detailed information).

Finally, we compared the performance measured on P-wave segments of leads selected using the whole ECG and the leads selected with the P-wave only. Errors for the P-wave and ECG-selected leads with 8 leads in a real case were: $15.47 \mu V$ for method $1,18.25 \mu V$ for method 2 and $17.51 \mu V$ for the 12-lead ECG (see table 5.2 and figure 5.6). Errors for the P-wave and P-wave-selected were: $15.16 \mu \mathrm{V}$ for method $1,17.58 \mu \mathrm{V}$ for method 2 and obviously the results for the 12-lead ECG remain unaltered since the lead set does not change (see table 5.2 and figure 5.6).

\subsubsection{Discussion}

As it can be inferred from the previous section, with the methods proposed we can obtain lead sets which differ from one lead selection method to another and also from one patient set to another even for patients from the same group. 


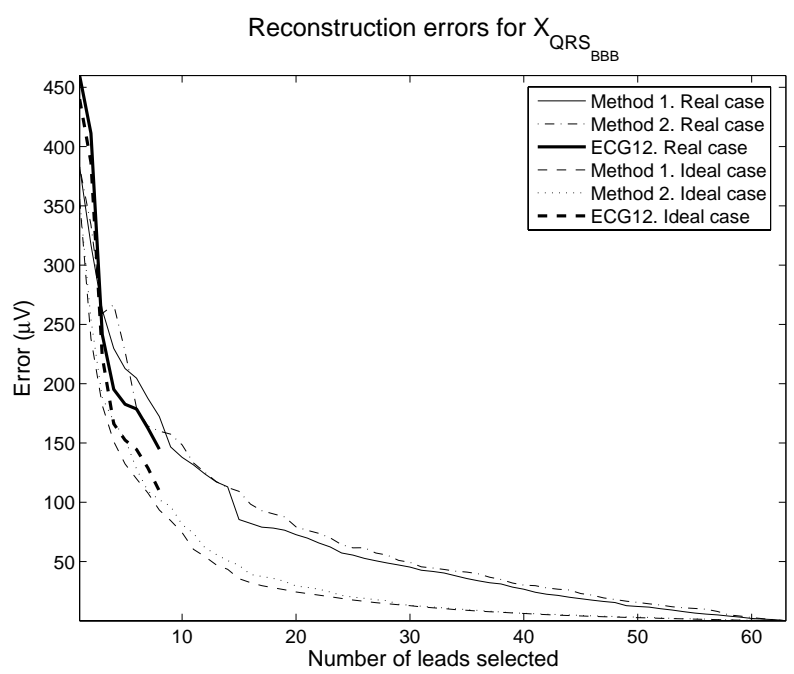

Figure 5.5: Error for limited lead sets on the QRS complex for the BBB group. Comparison of real and ideal case with both lead selection methods

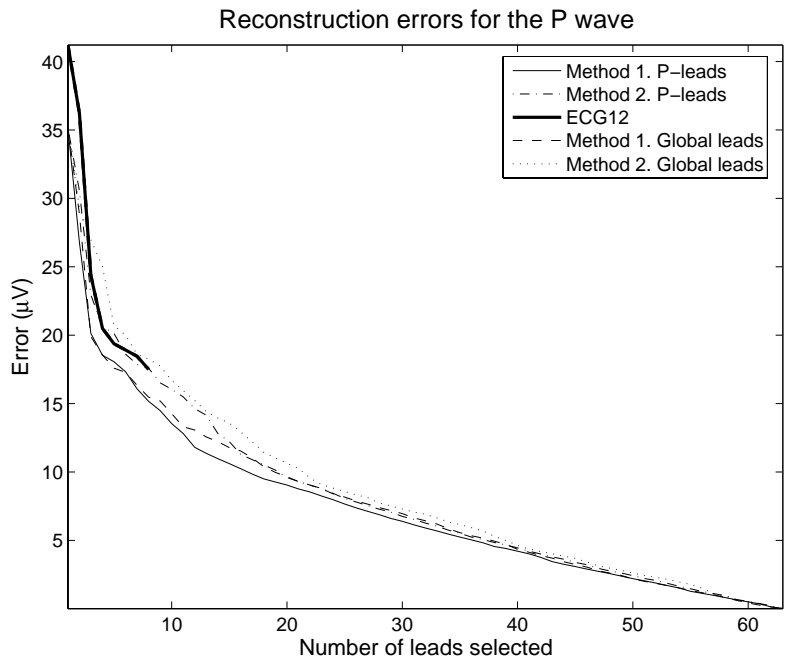

Figure 5.6: Error for limited lead sets on the $\mathrm{P}$ wave. Comparison of the performance of lead sets based on the whole ECG and the $\mathrm{P}$ wave. 
From the results shown in the previous section, it can be stated that the method 1 (i.e. Lux's method) is the one that achieves best performance in all groups and all ECG segments both for the real and the ideal case, whereas with the leads from method 2 and subsets of the 12-lead ECG the error values are slightly higher.

Error values obtained in the ideal scenario would reflect the lower bounds that can be achieved by each of the methods. Nevertheless, the performance decreases significatively under a real scenario, where the optimum lead set and the optimum reconstruction matrix for the patient under analysis are unknown, and hence are estimated from the study database. In this case, the performance present more variability among different scenarios than among lead selection methods.

This performance decrease in the real case is due to two causes: (1) the lead selection is not the optimum for a given patient or group of patients and (2) the linear matrix that reconstructs non-selected leads from selected leads is not the optimum one as well. In order to evaluate the influence of each cause on the overall performance, additional scenarios involving all possible combinations should be studied (i.e. lead selection over study dataset, but reconstruction matrix and performance measurement over test dataset and reconstruction matrix over study dataset, but lead selection and performance measurement over test dataset), which are out of the scope of this manuscript. Nevertheless, the fact that in some experiments the error is not monotonically decreasing as the number of leads increases leads us to conclude that the main reason is due to the reconstruction matrix, which is different for each patient (otherwise, the error could not be higher as a new lead is added to the lead selection).

An interesting observation is that although the 12-lead ECG does not reach the bounds of method 1, the 12-lead ECG is probably the lead system whose performance becomes more regular in a real case. These results suggest us that the variability of the reconstruction matrix when several patients are evaluated is not homogeneous for all lead subsets, and that not all leads may be adequate, even if they are chosen by the lead selection algorithms. In that sense, it can be pointed out that in order to determine an appropriate lead system, the optimum lead set of the study dataset must be derived with the restriction of not including leads that present high variability in their weights to reconstruct the rest of the leads. In addition, the satisfactory results that are obtained with the 12-lead ECG suggest the appropriateness of the standard 12-lead ECG in opposition to other studies that suggest repositioning of the electrodes.

As observed, the performance in the group of patients suffering from BBB are much poorer than with the rest of the patient groups, probably because electrical activations in this arrhythmia exhibit more heterogeneous dipole directions than during normal ventricular activation. Similar results have been also observed in [72], where in patients suffering from BBB more leads were needed to contain 
the same information content than in patients with other pathologies.

One criterium for determinating the optimum number of leads for the reconstruction of body surface potentials could be derived from Figures 5.2-5.6 at the inflection point in the curve. Lead sets formed by less than six to nine electrodes do not contain most information of the potentials from the whole body surface while adding a single lead after this inflection point adds little information. However, if a limited number of electrodes are to be selected (e.g. the 9 electrodes of the 12-lead ECG), errors in reconstruction are still significant (around $50 \mu \mathrm{V}$ within the whole ECG, $15 \mu \mathrm{V}$ for the $\mathrm{P}$-wave and $100 \mu V$ for the QRS complex). From these results it can be derived that ECG signals can only be approximated, but not accurately reconstructed from a linear combination of a limited number of signals. Thus, in the case that diagnostic information is present at limited body surface locations, and at the level of several dozens of $\mu V$, this might be hidden unless a high number of electrodes are employed (e.g. using a BSPM system).

Finally, it should be remarked that when reconstructing the P-wave, errors obtained from lead sets calculated from the $\mathrm{P}$-wave are similar to the errors obtained using the lead selection from the entire ECG signal, probably because the directions of the dipole during atrial activation are similar to the directions during ventricular activation and also because of the similarities of leads selected for both cases. This result would confirm the usefulness of the 12-lead ECG not only to study the ventricular activity, but also to analyze the normal atrial depolarization, i.e. the P-wave. However, this result may not be accurately extrapolated to the reconstruction of the atrial activity in case of some supraventricular arrhythmias, such as atrial fibrillation or atrial flutter, where more heterogeneous patterns of the dipole may take place as it occurred with the BBB group.

\section{Study limitations}

Although performance measurements were based on the reconstruction of body surface potentials, this criterion has also some limitations and could be extended or redefined. Indeed, the exact relationship between error and correlation values and the diagnostic information still remains unknown. A more comprehensive approach should include feature extraction and classification of cardiac arrhythmias, since specific patterns with important clinical information may appear at certain locations, independently of their contribution in the reconstruction of the surface potentials. 


\subsection{Selection of ECG leads for AF}

\subsubsection{Materials and Methods}

\section{Study Population}

One minute BSPM recordings were obtained from 17 patients admitted in the OVGUH and diagnosed with persistent AF (see section 3). Also, 10-second recordings of 18 healthy subjects were included in our study as control group.

\section{Preprocessing}

Signals were band-pass filtered with a passing band between 0.7 and $70 \mathrm{~Hz}$ in order to reject noise while preserving the signal content of ECG signals [121]. Then, QRS peaks were detected by using a modified version of Tompkins algorithm [104]. Detections were visually inspected in order to avoid incorrect detections of the algorithm. An averaged beat was calculated by template matching averaging, with a window size of $30 \%$ of the mean RR duration before the QRS peak and 70\% of the mean RR duration after the QRS peak. Only beats with a correlation index with the median beat that exceeded the threshold of 0.97 were considered for averaging.

Initial and final points of the QRS complex ( $\left.\mathrm{QRS}_{\text {onset }}, \mathrm{QRS}_{\text {offset }}\right), \mathrm{T}$ wave $\left(\mathrm{T}_{\text {offset }}\right)$ and $\mathrm{P}$ wave $\left(\mathrm{P}_{\text {onset }}, \mathrm{P}_{\text {offset }}\right)$ were detected in the averaged beat when present in the recording by using a polyline splitting based fiducial point detector.

QRS complexes were defined as starting on the first $\mathrm{QRS}_{\text {onset }}$ and ending on the last $\mathrm{QRS}_{\text {offset }}$. Remaining baseline offset was reduced by subtracting the mean value of each QRS complex in each lead. A total of 6 QRS complexes per patient in sinus rhythm were selected in order to have the same number of waves from each subject. These 6 QRS complexes were concatenated constituting the matrix $\left(\mathbf{X}_{\mathrm{QRS}}\right)_{64 \times 6 L}$, being $L$ the sample length of the QRS complex. The maximum amplitude value $\left(A_{\max }\right)$ of the matrix $\mathbf{X}_{\mathrm{QRS}}$ of each patient was calculated and then all the leads of that patient were divided by this $A_{\max }$ value, thus constituting the normalized matrix $\mathbf{X}_{\mathrm{QRSnorm}}$.

$\mathrm{P}$ waves were defined as beginning on the first $\mathrm{P}_{\text {onset }}$ and ending on the last $\mathrm{P}_{\text {offset }}$ P waves were again low-pass filtered with a cut-off frequency of $30 \mathrm{~Hz}$. A total of $6 \mathrm{P}$ waves of each healthy subject were concatenated and normalized as described for the QRS complexes thus constituting the matrices $\mathbf{X}_{\mathrm{P}}, \mathbf{X}_{\text {Pnorm }}$.

AF segments were defined as starting $25 \mathrm{~ms}$ after the last $\mathrm{T}_{\text {offset }}$ found in the 64 leads and ending $25 \mathrm{~ms}$ before the first $\mathrm{QRS}_{\text {onset }}$ detected. Detections were performed in the averaged beat and then applied before or after each QRS detection in ECG signal. Considering the frequency content of atrial fibrillatory signals [124], AF segments were again band-pass filtered between 2 and 30Hz. All AF segments longer than $250 \mathrm{~ms}$ of every patient were concatenated and constituted the $\left(\mathbf{X}_{\mathrm{AF}}\right)_{64 \times 6 L}$ matrix, where $L$ is the summed duration of all $\mathrm{AF}$ 
segments defined for a given patient and is dependent on the heart rate and QT duration. AF segments were normalized as previously defined for QRS complexes or $\mathrm{P}$ waves thus constituting the normalized matrix $\mathbf{X}_{\mathrm{AFnorm}}$.

\section{$\mathrm{P}$ wave and QRS complex datasets}

Patients belonging to the control group were divided into study and test datasets: the first half of patients constituted the study dataset while the second half of patients constituted the test dataset. Concatenation of normalized QRS complex potential matrices ( $\mathbf{X}_{\mathrm{QRSnorm}}$ ) of the first 9 patients

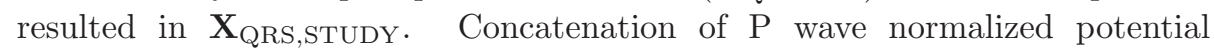
matrices $\left(\mathbf{X}_{\text {Pnorm }}\right)$ of the first 9 patients resulted in $\mathbf{X}_{\mathrm{P}, \mathrm{STUDY}}$.

\section{AF Datasets}

AF signals were divided into study and test datasets considering two different scenarios: experiment 1: nine patients included in the study dataset and eight patients in the test dataset; experiment 2: the first 30 seconds of the recording constituted the study dataset and the last 30 seconds of the recording constituted the test dataset.

Normalized potential matrices of patients belonging to the two defined study datasets were concatenated by taking the same number of samples from all patients. Concatenation of 7.5 seconds of $\mathbf{X}_{\mathrm{AFnorm}}$ matrices of the first 9 patients resulted in $\left(\mathbf{X}_{\mathrm{AF}, \mathrm{STUDY} 1}\right)_{64 \times L}$, where $L=9 \cdot \mathrm{Fs} \cdot 7.5$. Concatenation of 3 seconds of $\mathbf{X}_{\mathrm{AFnorm}}$ matrices belonging to the first 30 seconds of the recording of each patient resulted in the matrix $\left(\mathbf{X}_{\mathrm{AF}, \mathrm{STUDY} 2}\right)_{64 \times L}$, where $L=17 \cdot \mathrm{Fs} \cdot 3$.

\section{Lead selection}

Leads were selected according to iterative selection criteria previously established by R. Lux [88] (see section 2.4.1). Although this algorithm was proposed in an early study, it has been recently shown to be still superior to other novel methods [37, 38].

This algorithm was applied on previously defined study datasets which were constructed by concatenating normalized potential matrices, namely $\mathbf{X}_{\mathrm{AF}, \mathrm{STUdy} 1}, \mathbf{X}_{\mathrm{AF} \text {,STUdY2 }}, \mathbf{X}_{\mathrm{P}, \mathrm{STUdy}}$ and $\mathbf{X}_{\mathrm{QRS} \text {,Study }}$. In order to compare the reproducibility in the selection of leads in the case of atrial fibrillation, the same algorithm was applied to the test datasets, although the performance was only computed with the lead system derived from the study dataset.

\section{Proposal of a new 12-lead system optimized for AF}

A non-standard 12-lead set optimized for AF was calculated by first selecting the limb leads plus two precordial leads and then obtaining the remaining optimum 
4 leads by using the method described above. This lead system would allow to use existing ECG machines by keeping the limb leads plus at least two standard precordial leads and changing the position of four electrodes. Two cases were considered: constraints 1 implied selecting $V_{1}$ and $V_{2}$ and constraints 2 implied selecting $V_{1}$ and $V_{4}$.

\section{Reconstruction of body surface potentials}

Performance of a given lead set was quantified as the error in reconstruction of non-selected leads. Having the leads ordered as explained in Lead selection section, we calculated derived leads from an increasing number of selected leads as a linear combination of them. The linear combination that optimizes the derivation is calculated by Least Squares Optimization (see equation 5.2).

Normalized matrices for all study datasets, $\mathbf{X}_{\mathrm{AF}, \mathrm{STUdY} 1}, \mathbf{X}_{\mathrm{AF}, \mathrm{STUDY} 2}$, $\mathbf{X}_{\mathrm{P}, \text { STUDY }}$ and $\mathbf{X}_{\mathrm{QRS} \text {,STUDY }}$ were used for the computation of the transform matrices $\mathbf{T}_{\mathrm{AF} 1}, \mathbf{T}_{\mathrm{AF} 2}, \mathbf{T}_{\mathrm{P}}$ and $\mathbf{T}_{\mathrm{QRS}}$ respectively.

\section{RMS error in reconstruction}

The derived leads were compared to true recorded leads by the RMS error $\left(\varepsilon_{M}\right)$ as defined in equation 5.3.

This error was normalized according to voltage amplitude present in the dataset, and so a relative error was computed as follows:

$$
\begin{gathered}
A=\frac{\sum_{j=1}^{N} \sqrt{\frac{\sum_{i=1}^{S}\left(\mathbf{X}_{j, i}\right)^{2}}{S}}}{N} \\
\varepsilon_{R, M}=\frac{\varepsilon_{M}}{A},
\end{gathered}
$$

where $\varepsilon_{R, M}$ is the relative error of a lead set consisting of $\mathrm{M}$ leads.

In order to evaluate the performance of the algorithms, we carried out 2 experiments, corresponding to two different scenarios. The first scenario refers to an ideal situation where the lead selection and the transformation matrix $\mathbf{T}$ are applied over the same ECG signals, that is, the study data set. This would reflect the maximum performance that can be achieved by the lead selection algorithm because the optimum lead system and transformation matrix are available for the ECG signals under evaluation. The second scenario refers to a real situation where neither the optimum lead system nor the optimum transformation matrix for the ECG signals under test is available, that is, the test data set. In this case, the lead system and transformation matrix are computed for the study data set while the test dataset is used for evaluation.

Absolute and relative errors were computed for all the datasets described above and for both real and ideal situations. 
Same evaluations were performed on a lead selection consisting only on the eight independent leads of the standard ECG in order to compare their performance with the first eight leads selected with the algorithm proposed in [88]. Standard leads were ordered following the sequence: I, II, $V_{1}, V_{3}, V_{5}, V_{2}$, $V_{6}, V_{4}$.

\section{Spectral analysis of original and derived AF waves}

Atrial frequency is a parameter used in the diagnosis of AF. We complemented our analysis of the performance in reconstruction of non-selected leads by including the computation of derived spectral parameters. Derived leads were compared to true recorded leads in terms of their spectral content. In order to obtain the power spectral content of AF waves, atrial fibrillatory signals were estimated from the surface ECG by cancelling the ventricular activity from the subtraction of a matching QRS-T template in 3 segments of 10 seconds. This template was computed for each beat as a linear combination of the principal components previously obtained from the analysis of all beats in the ECG segment [18]. Welch's periodogram was used to obtain the power spectral density $\left(P_{A A}\right)$ of atrial signals (hamming window of 2.5 seconds and $50 \%$ overlap). The dominant frequency $\left(F_{A A}\right)$ was defined as the dominant peak in the power spectrum that accounts for at least $30 \%$ of the total power spectra. Power spectra of AF signals in which the cancellation process has not been successful present frequency components corresponding to the remaining ventricular activity and thus a dominant frequency cannot be determined.

Accuracy in the estimation of AF frequency was evaluated for the 12-lead ECG and for the proposed lead systems. Leads that did not belong to the lead system under study were used for evaluation. $F_{A A}$ and $P_{A A}$ were computed in the true recorded ECG signals and also in ECG signals reconstructed by using each lead set. Only leads in which a dominant frequency could be estimated in all lead systems under consideration were considered for comparison. Difference in the estimated $F_{A A}$ computed for the recorded signal and the estimated signal $\left(\Delta F_{A A}\right)$, correlation among estimated $F_{A A}$ in the recorded and estimated signals $\left(\rho F_{A A}\right)$ and correlation of the power spectra of recorded and estimated signals $\left(\rho P_{A A}\right)$ were computed.

\section{Statistical analysis}

Errors in reconstruction were computed for each patient and lead selection and then mean error values and standard deviations were computed. Comparisons between different lead selections evaluated for the same group of patients was obtained with a paired sample t-test. Comparisons between performances of lead selections applied to different groups of patients were obtained with an independent sample t-test. Two significance values were considered: $\mathrm{p}<0.05$ and $\mathrm{p}<0.01$. Differences of $\mathrm{p}$ value $>0.05$ were considered as non significant 
(ns).

\section{Limitations due to low signal-to-noise ratio}

When dealing with the information content of AF signals it must be taken into account that the signal-to-noise ratio of the fibrillatory waves is much lower than in QRS complexes. For this reason, the accuracy in the reconstruction of potentials can be limited by the noise level. Indeed, once the reconstruction error reaches the noise level, it can not be expected an improvement in the reconstructed potentials if a new lead is added to the reduced lead set. In order to estimate the noise level present we assumed that after having included almost all leads for the derivation on non-selected leads, the improvement due to the inclusion of a new lead is only caused by the inclusion of the noise of that lead. Therefore, the noise level is estimated as the tendency line of the error curve. The slope of this tendency line was calculated by using the last five points of the curve. An error of reconstruction of the maximum quantization error $(0.5$ $\mu \mathrm{V})$ above the noise level will be considered to be the limit in the information content achievable.

\subsubsection{Results}

\section{Leads selected}

The first eight leads selected for the study and test datasets can be observed in Figure 5.7, both for experiments 1 (division of patients into study and test groups) and 2 (seconds 0 to 30 of all patients constituted the study group whereas seconds 30 to 60 constituted the test group). Panel A shows different lead placements for the two groups of patients, whereas Panel B shows no difference in the selection of leads when applied to the same group of patients but in different time intervals.

Figure 5.8 shows the first 23 leads selected for the study dataset focused in the reconstruction of atrial potentials during AF. New 12 leads proposed for the study of AF can be observed in Figure 5.9 both for constraints 1 (limb leads plus V1 and V2) and constraints 2 (limb leads plus $V_{1}$ and $V_{4}$ ). Lead placements obtained in the different experiments include a high density of electrodes around $\mathrm{V} 1$ and the right part of the anterior torso, some electrodes on the precordial area and a few electrodes on the posterior torso.

\section{Performance of the leads selected}

Errors in reconstruction of the potentials on the whole body surface from a limited number of leads are depicted in Figure 5.10. RMS errors for both the ideal case and the real scenarios are represented. Both absolute and relative errors obtained from the first eight leads selected, applied on the derivation of QRS complexes, $\mathrm{P}$ waves and atrial fibrillatory waves corresponding to 
Anterior

\section{A. Experiment 1}

+ Study set

o Test set
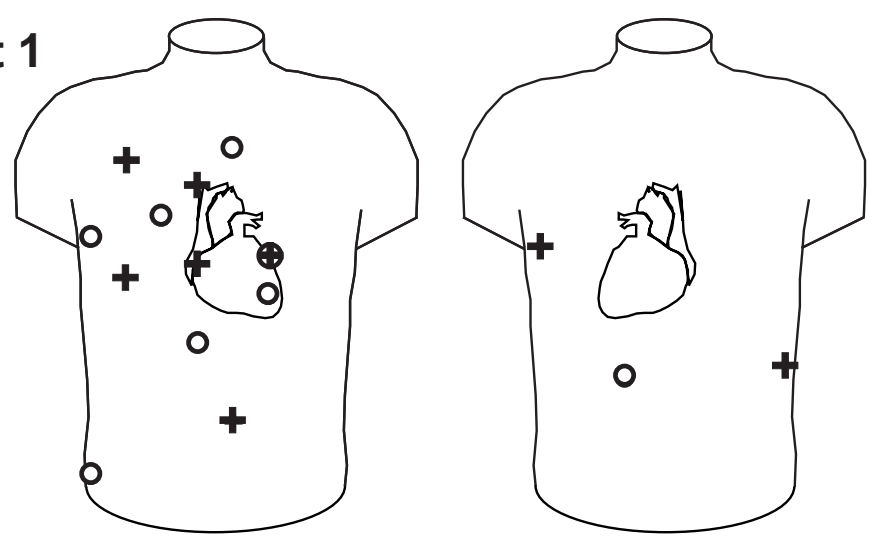

B. Experiment 2

+ Study set O Test set
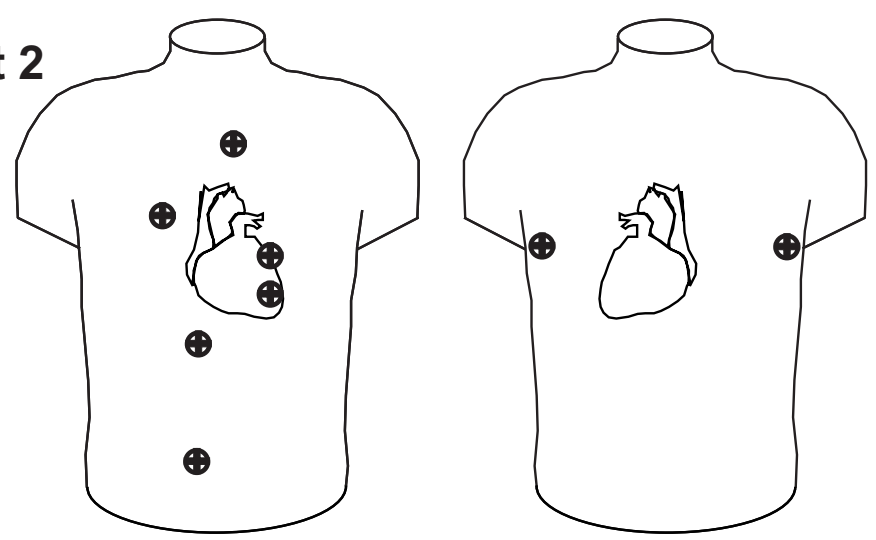

Figure 5.7: First eight leads selected for the study and test datasets. Panel A shows the leads selected in experiment 1 (patient division). Panel B shows the leads selected in experiment 2 (time division). Plus sign represents leads selected for the study dataset. Circles represent leads selected for the test set. 
Anterior

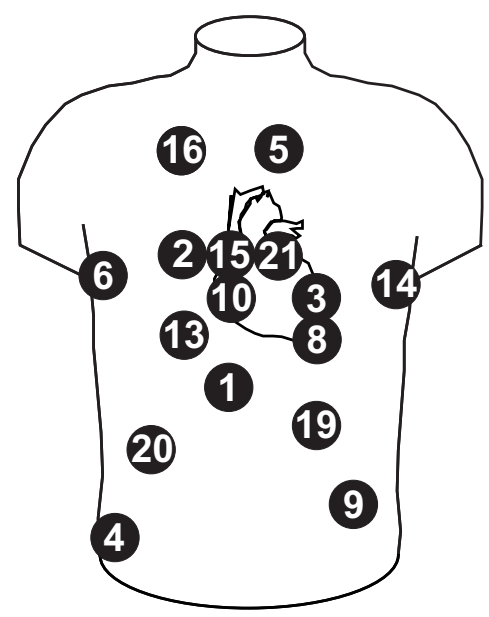

Posterior

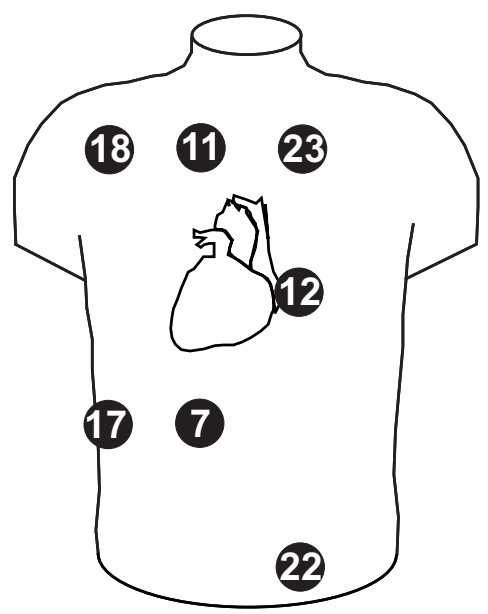

Figure 5.8: First twenty-three leads selected for the study dataset. The number of each lead positioning represents the order in which any given lead was selected.

experiment 1 (division of patients into study and test set) are summarized in Table 5.5. Errors in wave derivation considering an ideal scenario in which the lead set and transformation matrix is computed for the same signals under test are lower than errors in a more real scenario in which both lead set and transformation matrices are computed for the study set and applied on the test set $(31.8 \mu \mathrm{V}$ vs. $59.3 \mu \mathrm{V}, \mathrm{p}<0.01$, for the derivation of the QRS complex with the standard selected leads). This difference between results for real and ideal scenarios does not apply when the division of datasets is performed over time (see Figure 5.10.D).

Although error values are higher for waves with high amplitude than for waves with low amplitude such as fibrillatory waves, relative errors in the derivation of atrial fibrillatory waves are higher than those achieved in the derivation of other ECG waves (see Figure 5.11). Errors in reconstruction from the standard ECG in a real scenario are comparatively higher for AF waves than for the QRS complex: $52.8 \%$ vs. $25.7 \%(\mathrm{p}<0.01)$ or the $\mathrm{P}$ wave: $52.8 \%$ vs. $39.4 \%(\mathrm{p}=\mathrm{ns})$. This error in reconstruction of AF waves is reduced when the first 8 leads selected are considered instead of the standard ECG: $42.2 \%$ vs. $52.8 \%(\mathrm{p}<0.01)$. Improvement of the first 8 leads selected over the standard ECG is less significant when applied to the QRS complex (22.1\% vs. $25.7 \%, \mathrm{p}=\mathrm{ns})$ or the $\mathrm{P}$ wave $(33.0 \%$ vs. $39.4 \%, \mathrm{p}=\mathrm{ns})$.

According to the results from Figure 5.11 and Table 5.5, performance of reduced lead sets in AF is not as high as that in the QRS complex and the 
Anterior

\section{A. Constraints 1}

+ Study set
O Test set
- Fixed

B. Constraints 2

+ Study set

O Test set

- Fixed
Posterior
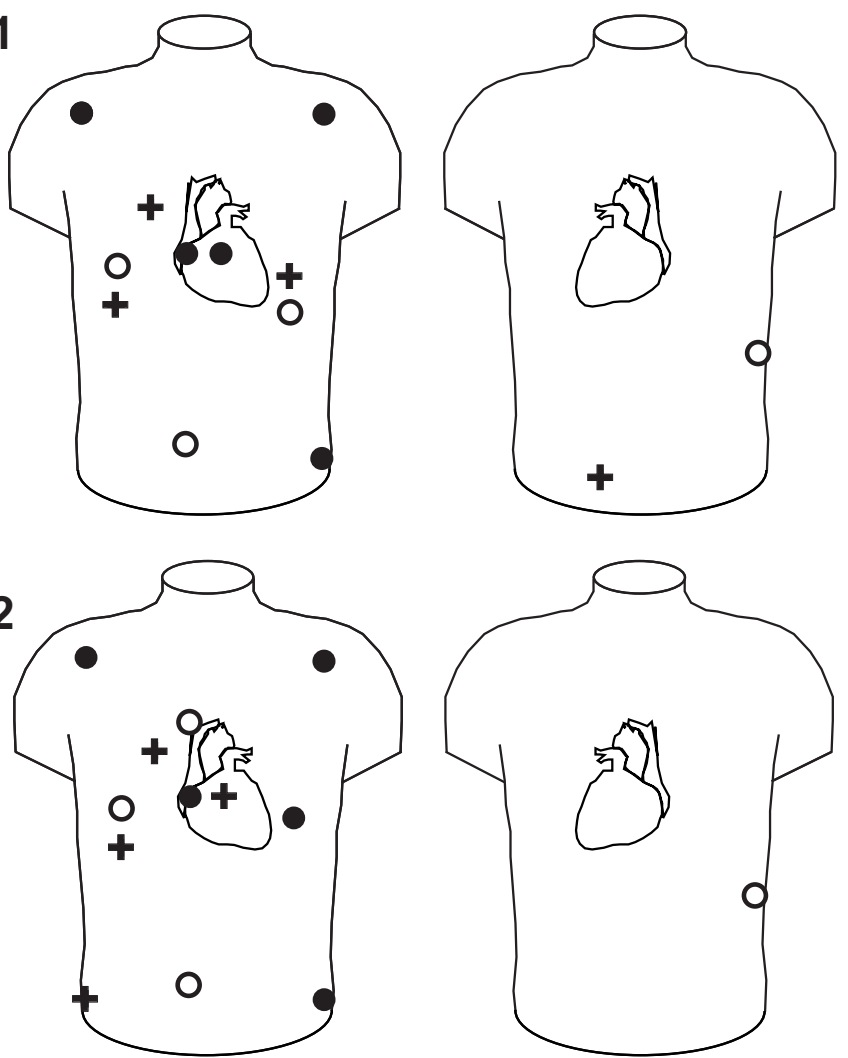

Figure 5.9: Twelve lead system proposed for AF. Panel A shows the leads selected with constraints 1 (selection of limb leads, V1, V2 and 4 calculated leads). Panel B shows the leads selected with constraints 2 (selection of limb leads, V1, V4 and 4 calculated leads). Plus sign represents leads selected for the study dataset. Circles represent leads selected for the test set. 


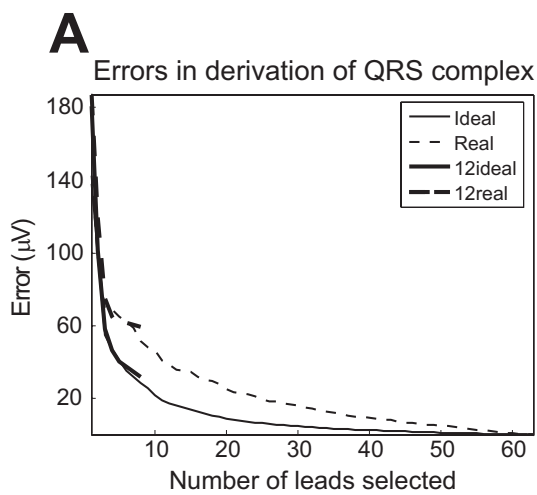

\section{B}

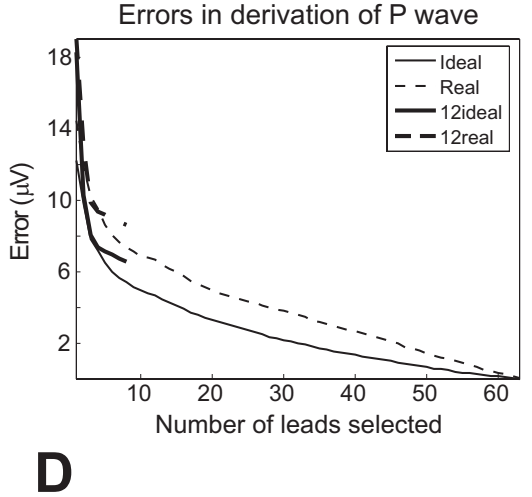

Errors in AF derivation - Experiment 1

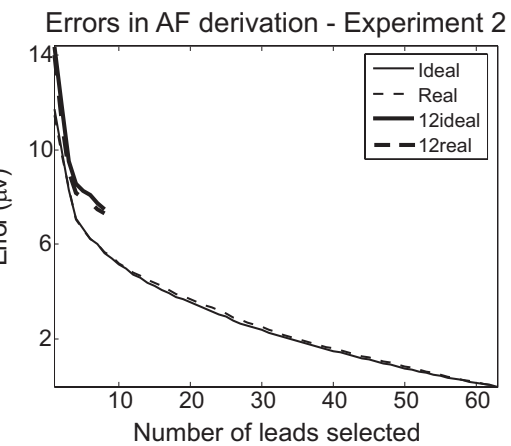

Figure 5.10: Errors for limited lead sets and standard ECG in the derivation of QRS complex, P wave and atrial fibrillatory waves. Solid line represents the results for the real case (leads and transfer matrices calculated for the study set and evaluation performed for the test data set) while dashed line represents the results for the ideal case (leads and transfer matrices and calculated for the study set and errors calculated for the test data set). Thick lines represents errors in reconstruction achieved with the standard 12-lead ECG while thin lines represent errors in reconstruction achieved with computed optimum lead sets. Notice that tracings corresponding to the 12-lead ECG stop at 8 independent leads. Panel A presents errors in microvolts in the derivation of the QRS complex. Panel B presents errors in microvolts in the derivation of the $\mathrm{P}$ wave. Panel $\mathrm{C}$ presents errors in microvolts in the derivation of atrial fibrillatory waves in experiment 1 (patient division). Panel D presents errors in microvolts in the derivation of atrial fibrillatory waves in experiment 2 (time division). 


\begin{tabular}{|c|c|c|c|c|c|}
\hline & & & $\begin{array}{c}\text { QRS complex } \\
\text { mean } \pm \text { SD }\end{array}$ & $\begin{array}{c}\mathrm{P} \text { wave } \\
\text { mean } \pm \mathrm{SD}\end{array}$ & $\begin{array}{c}\mathrm{AF} \\
\text { mean } \pm \mathrm{SD}\end{array}$ \\
\hline \multirow{4}{*}{$\begin{array}{c}\text { Standard } \\
\text { ECG }\end{array}$} & Ideal & $\mu \mathrm{V}$ & $31.8 \pm 6.7$ & $6.6 \pm 2.3$ & $7.1 \pm 0.8$ \\
\hline & & $\%$ & $15.0 \pm 3.5$ & $24.8 \pm 4.7$ & $48.1 \pm 10.7$ \\
\hline & Real & $\mu \mathrm{V}$ & $59.3 \pm 33.3$ & $8.6 \pm 3.6$ & $7.9 \pm 1.5$ \\
\hline & & $\%$ & $25.7 \pm 10.5$ & $39.4 \pm 19.4$ & $52.8 \pm 9.1$ \\
\hline \multirow{4}{*}{$\begin{array}{c}\text { First } \\
\text { eight } \\
\text { selected } \\
\text { leads }\end{array}$} & Ideal & $\mu \mathrm{V}$ & $28.1 \pm 6.8$ & $5.4 \pm 1.8$ & $5.3 \pm 0.5$ \\
\hline & & $\%$ & $13.1 \pm 2.4$ & $20.7 \pm 5.7$ & $36.7 \pm 9.8$ \\
\hline & Real & $\mu \mathrm{V}$ & $51.2 \pm 26.0$ & $7.3 \pm 4.0$ & $6.2 \pm 0.8$ \\
\hline & & $\%$ & $22.1 \pm 7.3$ & $33.0 \pm 17.7$ & $42.2 \pm 9.6$ \\
\hline
\end{tabular}

Table 5.5: Errors for the standard ECG and for the first eight selected leads in the derivation of QRS complex, $\mathrm{P}$ wave and atrial fibrillatory waves. Errors and relative normalized errors are presented both for the ideal and the real case. Errors for AF derivation correspond to experiment 1.

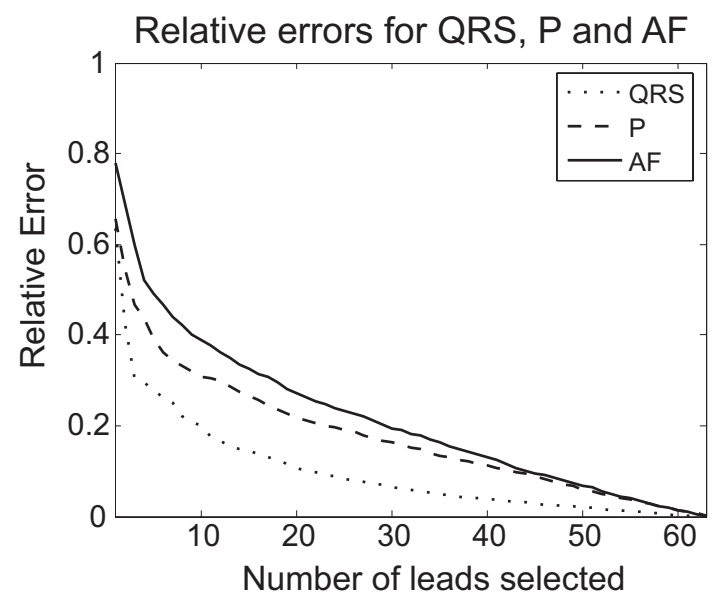

Figure 5.11: Relative errors for limited lead sets and standard ECG in the derivation of QRS complex, $\mathrm{P}$ wave and atrial fibrillatory waves. Solid line represents the results for $\mathrm{AF}$, dashed line represents the results for the $\mathrm{P}$ wave and dotted line represents the errors for the QRS complex. Only values corresponding to the real case are depicted. For AF, values correspond to experiment 1 (patient division). Panel A presents relative errors on nonnormalized matrices while Panel B presents the relative errors on the normalized matrices. 
number of leads needed to achieve a comparable performance needs to be increased. However, the amount of meaningful information in the case of AF signals has to be considered. The performance to be achieved should not have as an objective the reconstruction of potentials below the noise level. Figure 5.12 shows the errors in reconstruction of AF signals compared to the estimated noise level. From Figure 5.12 it can be observed that errors in reconstruction with more than 34 leads appear below the information content limit that we set $0.5 \mu \mathrm{V}$ above the noise level, which is the maximum quantification error. Consequently, inclusion of more than 34 leads does not increase the information content related to $\mathrm{AF}$ of a lead system. By computing this limit also for the QRS complex and the $\mathrm{P}$ wave we observed that the noise level is reached with 56 leads for the QRS complex and 17 leads for the $\mathrm{P}$ wave.

\section{Errors in AF derivation vs noise level}

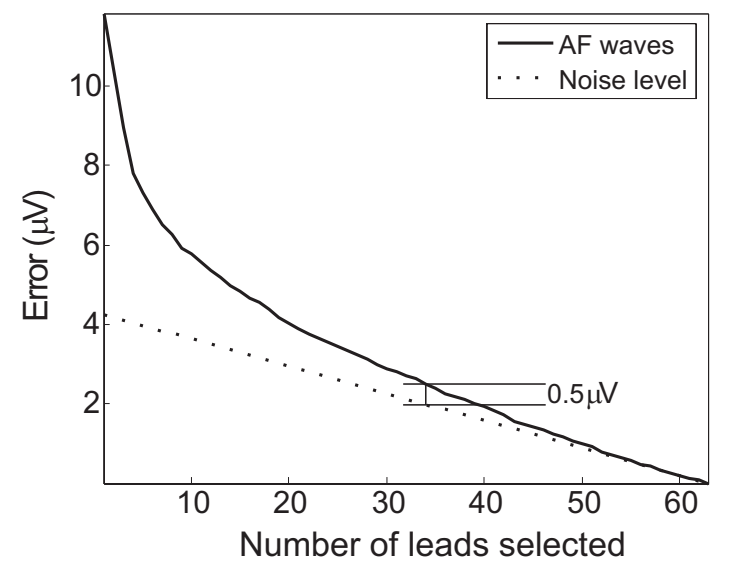

Figure 5.12: Errors in the derivation of atrial fibrillatory waves versus errors in reconstruction equal to a measured noise level. Noise level was computed on TP segments of healthy subjects. For AF, values correspond to experiment 1 (patient division) in the real case (leads and transfer matrices calculated for the study set and evaluation performed for the test data set).

Table 5.6 summarizes the number of leads required for reaching a given relative error threshold. While 8 leads are enough for reaching an error lower than $25 \%$ in the QRS complex, 17 leads are needed for the $\mathrm{P}$ wave and 23 are needed for atrial fibrillatory waves. If we lower this threshold down to $10 \%, 22$ leads are needed for the reconstruction of the QRS complex while the number of leads required both for the reconstruction of the $\mathrm{P}$ wave and $\mathrm{AF}$ exceed the number of leads with meaningful information given by the noise level present. An objective of $25 \%$ error is probably the most reasonable limit that we can set 
and 23 leads are needed to reach this limit in AF while 17 leads are needed for the $\mathrm{P}$ wave.

\begin{tabular}{c|ccc} 
& QRS complex & P wave & AF \\
\hline $25 \%$ & 8 & 17 & 23 \\
$10 \%$ & 22 & 43 & 45
\end{tabular}

Table 5.6: Number of leads needed in a real case to achieve a given relative error for the QRS complex, $\mathrm{P}$ wave and atrial fibrillatory waves. Values are given for the real scenario and relative normalized error thresholds of $25 \%$ and $10 \%$. AF values are given for experiment 1 .

Performance of the 12-lead ECG system and the proposed leads in the estimation of spectral features of non-recorded signals is presented in Table 5.7. A dominant frequency could not be estimated in $4.1 \pm 7.1$ true recorded leads. Additionally, a dominant frequency could not be estimated in $2.6 \pm 4.6$ leads for the standard ECG, $2.3 \pm 3.4$ leads for the first 8 selected leads and $1.5 \pm 2.5$ for the first 23 selected leads. Only leads in which a dominant frequency could be estimated in all lead systems were considered. Dominant frequency of derived AF signals showed a correlation $\left(\rho F_{A A}\right)$ with recorded AF signals of $0.57(\mathrm{p}<0.01)$ for the standard 12-lead ECG, $0.67(\mathrm{p}<0.01)$ for the first 8 selected leads and $0.76(\mathrm{p}<0.01)$ for the first 23 selected leads. Difference in the estimated frequencies $\left(\Delta F_{A A}\right)$ for leads derived from the 12-lead ECG was $0.6 \mathrm{~Hz}$ vs $0.5 \mathrm{~Hz}$ for the first 8 selected leads $(\mathrm{p}<0.01)$ and $0.3 \mathrm{~Hz}$ for the first 23 leads $(\mathrm{p}<0.01)$. Correlation of the spectra $\left(\rho P_{A A}\right)$ was 0.95 for the 12-lead ECG vs. 0.97 for the first 8 selected leads $(\mathrm{p}<0.01)$ and 0.98 for the first 23 selected leads $(\mathrm{p}<0.01)$.

\section{Performance of a new 12-lead system optimized for AF}

Results on the performance of the two proposed 12-lead systems that include the limb leads plus two standard precordial leads are summarized in Table 5.8. Both resulting lead sets (depicted in Figure 5.9) achieve comparable errors to those obtained with the first eight selected leads: 6.4 to $6.5 \mu \mathrm{V}$ or 43.0 to $44.1 \%$ error against $6.2 \mu \mathrm{V}$ or $42.2 \%$ ( $\mathrm{p}=\mathrm{ns})$ and better results than those obtained for the 12-lead ECG: $7.9 \mu \mathrm{V}$ or $52.8 \%(\mathrm{p}<0.05)$. Performance of any of these lead systems proposed for AF on the QRS complex and on the $\mathrm{P}$ wave is comparable to the performance of the standard 12-lead ECG: $22.2 \%$ to $24.8 \%$ error vs. $25.7 \%(\mathrm{p}=\mathrm{ns})$ for the QRS complex and $34.1 \%$ to $34.2 \%$ error vs. $39.4 \%(\mathrm{p}=\mathrm{ns})$ for the $\mathrm{P}$ wave.

\subsubsection{Discussion}

The standard 12-lead ECG is not optimized for the reconstruction of body surface potentials during AF. In fact, its reconstruction errors are not 


\begin{tabular}{c|cccc} 
& $\begin{array}{c}\text { N discarded } \\
\text { mean } \pm \mathrm{SD}\end{array}$ & $\rho F_{A A}$ & $\Delta F_{A A}(\mathrm{~Hz})$ & $\rho P_{A A}$ \\
& & mean $\pm \mathrm{SD}$ & mean $\pm \mathrm{SD}$ \\
\hline Standard ECG & $2.6 \pm 4.6$ & $0.57^{* *}$ & $0.6 \pm 0.9$ & $0.95 \pm 0.05$ \\
First 8 leads & $2.3 \pm 3.4$ & $0.67^{* *}$ & $0.5 \pm 0.9$ & $0.97 \pm 0.05$ \\
First 23 leads & $1.5 \pm 2.5$ & $0.76^{* *}$ & $0.3 \pm 0.8$ & $0.98 \pm 0.04$ \\
& & & & \\
& & & & \\
& & &
\end{tabular}

Table 5.7: Spectral measurements in derived AF signals compared to true recorded AF signals. Measurements were obtained for all leads not belonging to any of the lead systems under evaluation in 3 segments under evaluation for the test group in experiment 1 (division of patients into study and test groups). Number of leads per segment in which a dominant frequency could be estimated in the recorded signals but could not be estimated in the derived leads ( $\mathrm{N}$ discarded), correlation among the estimated atrial dominant frequencies $\left(\rho F_{A A}\right)$, difference in the estimated frequencies $\left(\Delta F_{A A}\right)$ and correlation of the spectra are given $\left(\rho P_{A A}\right)$.

\begin{tabular}{|c|c|ccc|}
\hline \multicolumn{2}{|c|}{} & $\begin{array}{c}\text { QRS complex } \\
\text { mean } \pm \mathrm{SD}\end{array}$ & $\begin{array}{c}\mathrm{P} \text { wave } \\
\text { mean } \pm \mathrm{SD}\end{array}$ & $\begin{array}{c}\text { AF } \\
\text { mean } \pm \mathrm{SD}\end{array}$ \\
\hline Standard & $\mu \mathrm{V}$ & $59.3 \pm 33.3$ & $8.6 \pm 3.6$ & $7.9 \pm 1.5$ \\
ECG & $\%$ & $25.7 \pm 10.5$ & $39.4 \pm 19.4$ & $52.8 \pm 9.1$ \\
\hline First eight leads & $\mu \mathrm{V}$ & $57.1 \pm 27.9$ & $7.7 \pm 4.4$ & $6.2 \pm 0.8$ \\
selected for AF & $\%$ & $24.8 \pm 8.3$ & $34.7 \pm 19.7$ & $42.2 \pm 9.6$ \\
\hline New 12-lead system & $\mu \mathrm{V}$ & $51.5 \pm 26.9$ & $7.5 \pm 3.6$ & $6.5 \pm 0.8$ \\
Constraints 1 & $\%$ & $22.2 \pm 7.7$ & $34.2 \pm 18.1$ & $44.1 \pm 10.0$ \\
\hline New 12-lead system & $\mu \mathrm{V}$ & $53.2 \pm 30.0$ & $7.5 \pm 3.6$ & $6.4 \pm 0.8$ \\
Constraints 2 & $\%$ & $22.9 \pm 9.0$ & $34.1 \pm 18.1$ & $43.0 \pm 8.4$ \\
\hline
\end{tabular}

Table 5.8: Errors for the standard ECG, first eight leads selected for AF and proposed replacement of the standard leads in the derivation of QRS complexes, $\mathrm{P}$ waves and atrial fibrillatory waves. Errors and relative normalized errors are presented only for the real case and experiment 1 for AF (patient division). 
negligible: $53 \%$ error from our results. Also, computation of lead sets optimized for the study of AF results in lead placements that differ from the standard lead placements. Our results agree with previous works $[66,67]$ that suggested that the standard 12-lead electrocardiogram is not optimum for the study of atrial fibrillatory signals.

If we aim at defining a lead set optimized for AF, the first question that needs to be addressed is the number of leads that are necessary. Previous studies regarding the optimum number of leads using real BSPM data have dealt with the information content of non-fibrillating signals. Hoekema et al. [61] found that the number of independent signals in body surface maps is of the order of 10. However, these 10 independent signals may not be appropriately sampled by 10 electrodes according to the studies of Lux et al [88], who reported that 32 leads could reconstruct body surface potentials with $5 \%$ error. From our results, only 8 electrodes can reconstruct body surface potentials at 64 positions with $25 \%$ error for the QRS complex and 22 electrodes are needed in order to decrease this error down to $10 \%$, which is consistent with the observations made by Hoekema and Lux.

The number of independent signals in simulated AF was found to be of the order of 60 [67], multiplying by 6 the number of independent signals found in non-fibrillating BSPM recordings. In order to determine experimentally the number of leads needed to reconstruct body surface potentials during AF, we applied the lead selection procedure proposed by Lux et al. [88] and computed errors in reconstruction of body surface potentials in two different scenarios: (1) a "real" scenario in which the lead selection and the transform used for the reconstruction of non-selected leads is performed on a study dataset and the evaluation is performed on a test dataset and (2) an "ideal" scenario in which the evaluation is performed on the same dataset used for the guidance of the lead selection procedure and the computation of transform matrices. In order to extract conclusions about the number of leads needed we only considered the "real" scenario. However, we included this "ideal" scenario in order to separate two effects that have great influence in the evaluation of the performance of a lead set: (1) the nature of the signals under study which is related to the number of independent signals and the signal-to-noise ratio and (2) inter-patient variability which results in optimum lead placements and transform matrices which are different for every subject under study. In an ideal scenario the effect of patient variability is reduced and thus we can obtain results which are less dependent on inter-patient variability and reflect the effect of the inclusion of a given number of leads on the global reconstruction error which is primarily due to the nature of fibrillatory signals. However, in a real situation these two effects are not separable and we can only interpret the results arising from the "ideal" scenario as a limit of the best performance that can be achieved even if the transform matrix and lead selection could be performed by using patientspecific information. 
According to our results, 23 leads are needed in order to reconstruct body surface potentials during AF with $25 \%$ error, which is a large number compared to the 8 leads needed for the QRS complex and the same degree of accuracy. Although inclusion of more than 23 leads may reduce the reconstruction error in case of $\mathrm{AF}$, inclusion of more than 34 leads adds information below the noise level and thus their information content is limited. Also, 23 leads are better in order to accurately estimate the spectral content of AF signals than a smaller subset of leads, allowing a better estimation of the spectrum and the dominant frequency in non recorded leads. This finding can be easily attributed to the nature of fibrillatory signals: electrical activity in fibrillating atria is far from being organized or explained by a dipolar model as opposed to the QRS complex which is mainly dipolar. In those circumstances, fibrillatory waves recorded in a given location are hardly reconstructed from electrodes located far away from this location.

The location of these 23 leads may not be a crucial issue and many lead subsets -not all lead subsets- may present a similar performance. The first 23 leads that resulted of our experiment include right anterior electrodes and posterior electrodes. Two questions arose after we had computed this optimum lead set: (1) is this optimum lead set unique or does it vary among patients? and (2) is it stable over time or does it vary as AF signals do? In order to answer these two questions we set up two different experiments: in experiment 1 we divided our patients into two groups and in experiment 2 we put all patients together but studied separately two time intervals. We found that the optimum set of electrodes is not unique by observing the leads selected for our two subsets of patients in experiment 1. Also, we observed that the location of these electrodes is repeatable and stable: lead positioning is almost identical when ECG signals of the same patients acquired at different time instants are considered, as can be derived from our experiment 2 .

By stating that 23 leads are needed in order to reconstruct body surface potentials during $\mathrm{AF}$ we do not mean to imply that $\mathrm{AF}$ cannot be diagnosed with a more reduced number of leads. In fact, the existence of atrial fibrillation can be diagnosed by using an even more reduced set of leads. However, body surface potential maps during AF will only be at least as accurate as the 12lead ECG for the QRS complex (25\% error) if 23 leads are used. Interpretation of body surface maps during atrial fibrillation is still an open research field. However, preliminary studies performed by our group suggest that they can be a valuable non-invasive tool for the study of the state of the atria in patients with AF.

In case that only a small number of leads are available, a positioning of these leads which optimizes for the study of atrial fibrillatory signals can improve the amount of information that is recorded both in terms of the RMS error and in the ability of estimating the dominant atrial frequency in non-recorded leads. We present the results for different conditions: (1) no 
restrictions applied in the search procedure and (2) force the inclusion of limb leads plus two precordial leads in order to keep some ECG signals that the cardiologist is familiar with while using the same ECG machine that is available. In either case, selected leads include electrodes (1) on the right side of the torso (2) superior to $V_{1}$ and (3) on the posterior torso. These results agree with lead placements previously proposed for the study of $\mathrm{AF}[66,67]$ although the exact location of the electrodes is not identical. Errors in reconstruction of atrial fibrillatory waves from these limited lead sets are similar (42.2\% to $44.1 \%)$ and always improve the $52.8 \%$ error achieved with the standard 12-lead ECG. Performance of the standard 12-lead electrocardiogram is more optimized for the study of the QRS complex or the $\mathrm{P}$ wave. In fact, improvement after lead repositioning (3.6\% improvement for the QRS complex and 6.4\% improvement for the $\mathrm{P}$ wave) is not significant. The standard electrocardiogram may be adequately sampling these non-fibrillating signals that can be represented with some accuracy with a dipolar model. Any of the proposed locations for lead sets limited to 8 leads allow at least $8.7 \%$ less error (and up to $10.6 \%$ error)in reconstruction than the standard 12-lead ECG without detriment to the performance on the QRS complex or $\mathrm{P}$ wave. The clinical relevance of this $8.7 \%$ improvement is questionable and an increase of the number of exploring electrodes is needed if a reasonable accuracy is desired, but if this is not possible, a change in the position of the standard 12-lead ECG may allow some improvement in the study of atrial fibrillatory signals while the performance on other ECG waves is not altered.

\section{Study Limitations}

We have computed optimum lead sets based on an information index and sequential search algorithm described by Lux et al [88]. This method is based in an optimization of the error in reconstruction of leads not included in any lead set and not based in any clinical parameter. Also, this sequential search algorithm is suboptimal compared to exhaustive search algorithms that test all possible combination of leads for any number of leads [37]. This search strategy is computationally intensive and as their authors reported, results are not more satisfactory than lead sets obtained by using Lux's algorithm.

A main limitation of our study is the limited number of patients enrolled and limited length of our recordings, specially in the case of atrial fibrillatory signals that are variable in time. Consequently our results may not be valid for a different population of patients and are specific for our database. 


\subsection{Conclusions}

Two strategies for lead selection from BSPM recordings have been investigated. Although the criterion for lead selection was different in each method, the rationale was to choose, by using an iterative procedure, the lead that adds more information to the leads selected in the previous iteration. In general, errors in reconstruction of the surface potentials in a real case are significant, even if a high number of electrodes is considered, reflecting the existence of non-dipolar contents in the ECG. This problem can be accentuated in some pathological conditions where the activation does not follow a dipolar pattern, such as bundle branch block and specially in atrial fibrillation.

Nevertheless, in case a number of leads is to be selected, we consider that slight differences on lead positioning have little effect on the signal content of a group of patients, in which the most appropriate lead set may differ from one patient to another. Any reasonable lead selection method can be used for this purpose, although if one is to choose a lead selection method, the method proposed by Lux appears to be the best option.

Our results suggest that if a lead system is limited to 9 electrodes, then the 12-lead ECG is appropriate for the study of $\mathrm{P}$ waves and QRS complexes. However, limited lead sets do not allow an accurate reconstruction of all the electrical information available on the body surface in the case of atrial fibrillation. At least 23 leads are needed in order to have the same degree of accuracy as the 12-lead ECG for a normal QRS complex.

All these results suggest that the fibrillatory waves are significantly more heterogeneous than P-waves and QRS complexes. Accordingly, in order to characterize and analyze the atrial signal during AF, our recommendation would be to employ as many leads as possible (with up to 34 leads instead of using a reduced lead system), considering also the possibility of employing a BSPM recording system. Nevertheless, if a limited lead set is to be chosen, a repositioning of only four electrodes from the standard ECG improves the accuracy of the characterization of atrial fibrillatory waves, allowing a reconstruction of atrial fibrillatory signals with $10.6 \%$ less error than the error achieved with the standard 12-lead ECG. This repositioning of electrodes may include more right anterior electrodes and one posterior electrode. 


\section{Chapter 6}

\section{Non-invasive mapping of human $\mathrm{AF}^{1}$}

\author{
6.1 Introduction \\ 6.2 Materials and Methods \\ 6.3 Results
}

\author{
6.4 Discussion \\ 6.5 Conclusions
}

Results from previous chapters have evidenced the inability of the standard ECG for the extraction of spatial indicators of the propagation of AF waves. This limitation of the standard ECG is a consequence of the lack of dipolarity of atrial activation during AF. For a non-dipolar activation, potentials on each region of the body surface may reflect preferentially the local activation of the most proximal myocardial area. These local potentials may not be adequately sampled by the standard ECG but accurately reflected in BSPM recordings. In this chapter we evaluate the use of BSPM for the quantification of atrial organization during AF in a non-invasive manner. The low signal-to-noise ratio in AF signals together with the complexity of analyzing these chaotic activations has prevented the creation of BSPM maps during AF in the past. We have been able to generate and analyze BSPM maps by applying additional signal processing techniques and map display concepts never used before.

\footnotetext{
${ }^{1}$ Chapter based on the manuscript: M. S. Guillem, A. M. Climent, F. Castells, D. Husser, J. Millet, A. Arya, C. Piorkowski, and A. Bollmann. Non-invasive mapping of human atrial fibrillation. Journal of Cardiovascular Electrophysiology (accepted for publication).
} 


\subsection{Introduction}

Atrial fibrillation is a supraventricular arrhythmia in which the electrical activation shows an uncoordinated pattern. The surface electrocardiogram is a valuable tool not only for the diagnosis of $\mathrm{AF}[8,11,105]$ but also for better non-invasive characterization of the fibrillatory process. In particular, lead $V_{1}$ is the most commonly used lead, as fibrillatory waves appear with higher amplitude compared to other leads [9].

However, the 12-lead ECG offers a limited amount of information about the organization degree in the atria in an individual or for further understanding of the mechanisms maintaining AF in each patient. Optical mapping and modelling studies have shown that the uncoordinated electrical activity observed in $\mathrm{AF}$ can be maintained by anatomical or functional barriers which may provide a substrate for reentry $[2,69]$. Rotors, spiral waves and multiple wavelets have been observed in invasive mapping experiments and are thought to be important mechanisms for AF maintenance $[15,34,69,68,93,92,91]$. Although both invasive studies in animals and model studies are of main interest for the understanding of mechanisms underlying AF, they are not directly applicable into clinical practice.

Activation mapping in humans during AF revealed that different atrial activation patterns ranging from organized AF with single wavefronts to disorganized activity with multiple simultaneous wavefronts exist [71]. Previous non-invasive studies have attempted to increase the amount of information recorded in the standard electrocardiogram by replacing the electrodes into non-standard positions $[66,67]$. However, their advantage over the 12-lead ECG is limited because they do not provide information about mechanisms of AF or the degree of organization in the atria. Also, the vectorcardiogram has been proposed as a tool for the evaluation of atrial wavefronts $[102,131]$. However, the use of vectorcardiograms for AF characterization can be questioned by the fact that the electrical activity during $\mathrm{AF}$ is sometimes far from being a single wavefront or dipolar. Body surface potential mapping (BSPM) has been previously applied to the study of many cardiac diseases. Despite the advantages of body surface electrical maps over the conventional ECG, they have not been applied so far for the characterization of activation patterns during AF.

In this study, we present, for the first time, the potential of body surface maps obtained with a custom-made lead system for the non-invasive evaluation of activation patterns in patients with AF. 


\subsection{Materials and Methods}

\subsubsection{Study Population}

This study included 14 consecutive patients with persistent AF with electrode configuration 2 (see chapter 3). Their clinical and echocardiographic characteristics are summarized in Table 6.1. Baseline recordings were acquired in 10 patients for 4 minutes and in four patients for 2 minutes with the patients in a supine position after a 5 -minute resting period.

\begin{tabular}{|c|c|}
\hline Age, years & $68 \pm 14$ \\
\hline Male/female & $10 / 4$ \\
\hline AF duration, months & $12 \pm 18$ \\
\hline Hypertension & 10 \\
\hline Coronary artery disease & 4 \\
\hline Beta blocker & 10 \\
\hline Calcium channel blocker & 4 \\
\hline Digoxin & 5 \\
\hline LAD, mm & $46 \pm 6$ \\
\hline LVEF, $\%$ & $54 \pm 8$ \\
\hline
\end{tabular}

Table 6.1: Patient characteristics

\subsubsection{ECG Signal Processing}

ECG signals were processed using Matlab 7.0.1 (The Mathworks Inc, The Neatherlands). Baseline wandering was reduced by subtracting the baseline to the recording, calculated by using a 3rd order low-pass Chebyshev filter. Signals were low-pass filtered in order to avoid myoelectric interference by using a low-pass filter $(\mathrm{fc}=40 \mathrm{~Hz}$ ) and downsampled to $512 \mathrm{~Hz}$ in order to speed up the calculations.

Only long segments free from ventricular content were included in the study. For that purpose, the peaks of QRS complexes were detected in V1. RR intervals longer than $950 \mathrm{~ms}$ were first selected and a TQ segment was defined as starting $400 \mathrm{~ms}$ after the QRS peak and ending $150 \mathrm{~ms}$ before the next QRS peak (Figure 2). All TQ segments were visually inspected in order to avoid wrong detections or inclusion of $\mathrm{T}$ segments. After this step, only segments free from ventricular activity and longer than $350 \mathrm{~ms}$ were considered.

In order to avoid baseline wandering, all AF segments were filtered again. First, the mean value in each lead was subtracted and the baseline was again calculated and subtracted. For the estimation of the baseline, segments were downsampled $(\mathrm{fs}=128 \mathrm{~Hz})$ and low-pass filtered $(\mathrm{fc}=3.3 \mathrm{~Hz})$. Then, the baseline was upsampled to $512 \mathrm{~Hz}$ and subtracted to the segment. After baseline wandering correction, AF segments were low-pass filtered ( $\mathrm{fc}=20 \mathrm{~Hz}$ ). 


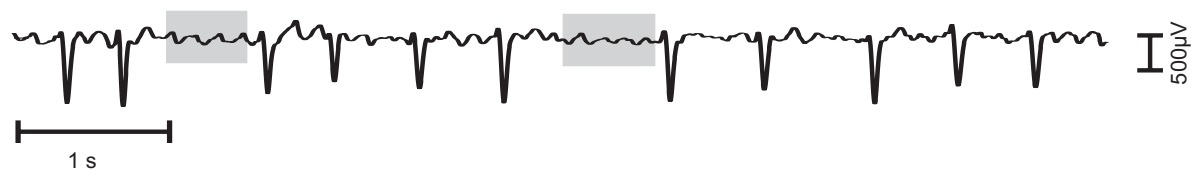

Figure 6.1: Selection of AF segments free from ventricular activity. Selected segments of lead V1 from patient 3 are highlighted on a 10-second fragment.

Notice that this band-pass filtering step is essential for the construction of surface AF maps given the low signal-to-noise ratio of AF signals and without this filtering, meaningful maps cannot be constructes.

All leads in all segments were visually inspected. Leads presenting noticeable noise contributions (typically, a transient loss of contact in one electrode) were discarded and interpolated from its neighboring electrodes by cubic spline interpolation. In order to prevent a critical loss of information by interpolating, segments presenting more than three discarded leads were excluded from study $(N=4)$. Number and duration of segments that were finally included in the study for each patient are summarized in Table 6.2. Notice that the number of segments included depend on the duration of RR intervals in each patient.

\begin{tabular}{cccc} 
& N segments & Total duration (s) & $\begin{array}{c}\text { Segment duration }(\mathrm{ms}) \\
(\text { mean } \pm \mathrm{SD})\end{array}$ \\
\hline Patient 1 & 8 & 3.84 & $456 \pm 32$ \\
Patient 2 & 97 & 51.15 & $548 \pm 144$ \\
Patient 3 & 13 & 7.24 & $628 \pm 136$ \\
Patient 4 & 103 & 62.16 & $632 \pm 140$ \\
Patient 5 & 10 & 4.62 & $468 \pm 20$ \\
Patient 6 & 103 & 66.42 & $640 \pm 228$ \\
Patient 7 & 39 & 16.28 & $418 \pm 97$ \\
Patient 8 & 141 & 75.85 & $584 \pm 205$ \\
Patient 9 & 80 & 36.73 & $471 \pm 64$ \\
Patient 10 & 85 & 38.62 & $453 \pm 122$ \\
Patient 11 & 69 & 27.76 & $416 \pm 90$ \\
Patient 12 & 189 & 110.33 & $560 \pm 173$ \\
Patient 13 & 25 & 9.08 & $397 \pm 106$ \\
Patient 14 & 71 & 27.56 & $382 \pm 63$
\end{tabular}

Table 6.2: Number and duration of AF segments selected for each patient. 


\subsubsection{Map display and wavefront propagation descriptors}

Once AF segments had been isolated and processed, maps for each time instant belonging to each segment were constructed. For that purpose, potentials at a given time instant were arranged in two matrices: one for the front $(5 \times 8)$ and one for the back $(4 \times 4)$. In such matrices, signals recorded from neighbouring electrodes are placed together. In order to obtain a smooth representation of the maps, finer matrices for the front $(50 \times 80)$ and the back $(40 \times 40)$ were created. Potentials for unknown positions were interpolated by cubic spline interpolation.

Two display modes were implemented: the isopotential mode and the wavefront propagation mode. In the isopotential mode, voltages at a given time instant were represented according to a color scale. A video can be generated of any segment as a succession of maps. In the wavefront propagation mode, the time series of the propagation of a single wave or multiple waves is summarized in a single map. A succession of lines connecting points on the surface with positive slope and a voltage equals to zero at any time instant is displayed. This can be interpreted as displaying the points on the surface that are perpendicular to the electric field generated by the wavefront. Notice that we only take those points with a positive slope in order to have a single representation of each wavefront instead of having both the forward and backward movement of the wavefront. The rationale to employ this criterion is that at these points the slope of the signal is maximum, and hence the trigger is more robust to noise. The isochrone map summarizes the information given in a video and allows a more comprehensive visualization of the propagation of wavefronts. Visual explanation of how these maps are calculated is given in Figure 6.2. The succession of wavefronts is represented using a color scale according to the time instant in which each wavefront appears. The time lag considered for representing each map was defined according to the activity observed, and so separation between maps was done when no zero-voltage line was detected.

Maps of all AF segments of each patient were generated and visually inspected. Wavefront propagation maps were classified into three different groups according to the depolarization pattern observed. We used the same criteria and terminology for classification as that of Konings et al. [71] although applied to surface recordings instead of electrograms. Type I includes those segments that present a single wavefront propagating across the body surface. Type II includes segments in which a single wavefront is observed, although it may suffer from blockage or splitting. Type III includes segments in which no clear wavefront can be observed or multiple wavefronts that do not propagate across the body surface can be observed simultaneously. 
1

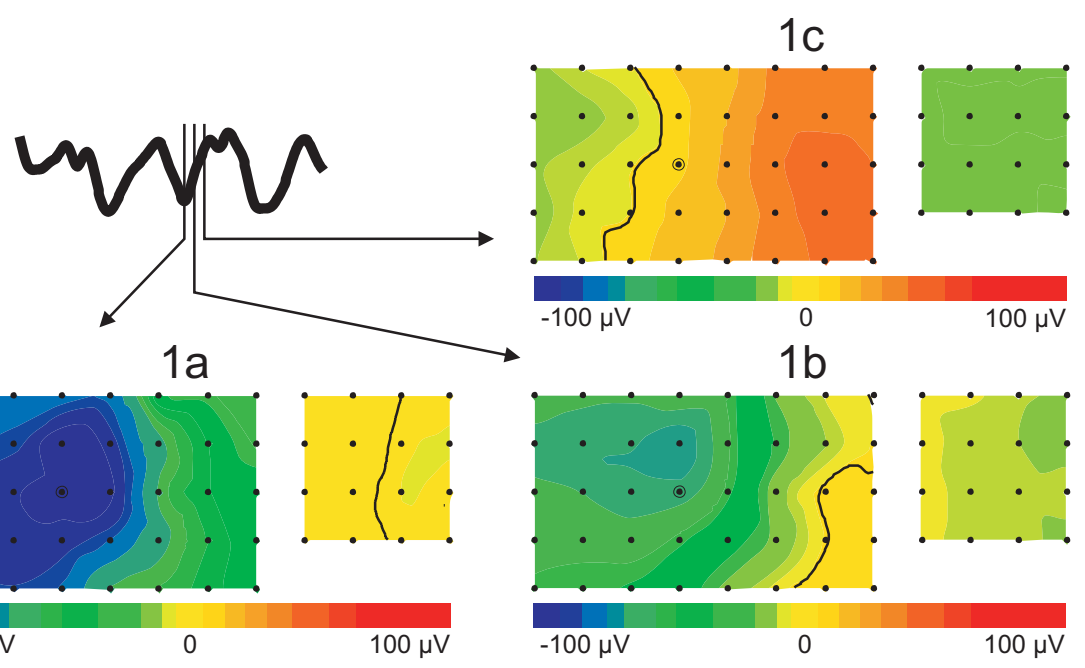

2
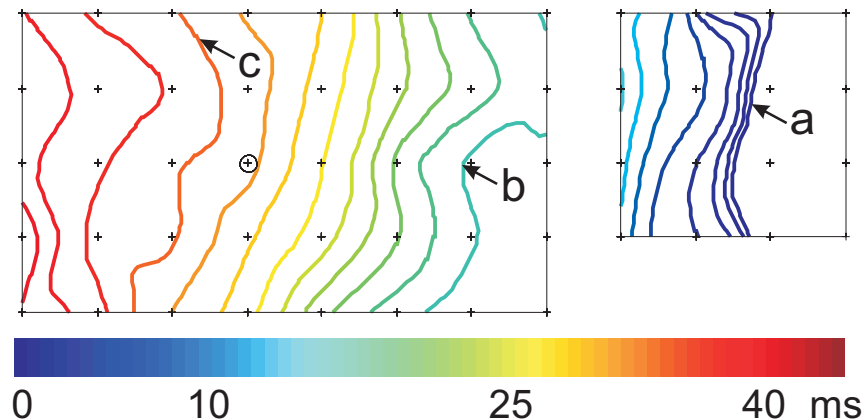

25

$40 \mathrm{~ms}$

Figure 6.2: Examples of isopotential and wavefront propagation modes. Panels 1a to 1c show isopotential maps at different time instants in an AF segment. The solid line connects points on the surface with zero voltage. Panel 2 shows the wavefront propagation map of the same segment. Wavefront propagation lines are drawn every $2 \mathrm{~ms}$. 


\subsubsection{Assessment of short-term reproducibility}

In order to assess the reproducibility in our measurements, minutes 1-2 and minutes 3-4 were analyzed separately. Percentage of time at which each type of wave is present was calculated both for minutes 1-2 and minutes 3-4. Each patient was classified into group 1, 2 or 3 depending on the predominant type of waves present. Patients were included into group 1 if they presented type-I activation during more than $50 \%$ of the time, into group 3 if type III activation was present more than $50 \%$ of the time and into group 2 otherwise. Comparison between classifications of patients in both periods of time was computed by Pearson correlation. A p value $<0.05$ was considered statistically significant. Relationship among the number of segments studied and their duration in each patient with the dominant AF type was assessed by an unpaired sample t-test.

\subsection{Results}

Four selected segments, representative of typical wavefronts that were observed, are depicted in Figure 6.3. Two segments were selected from patient 3 with a difference in time of $2 ' 39$ ". One segment was selected from both patient 5 and patient 6. Existence of AF and exclusion of ventricular activity in the segments under study can be confirmed.

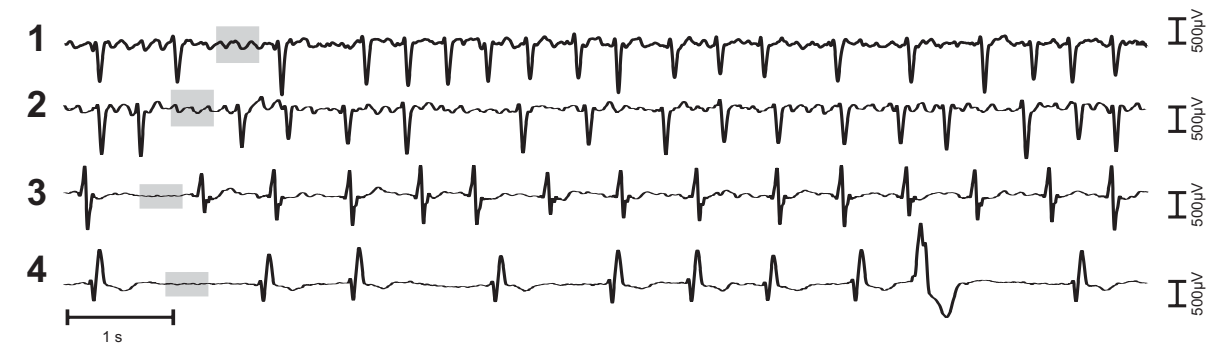

Figure 6.3: 10-second recordings showing the same AF segments as in Figures 6.4 and 6.5 (highlighted). Panels 1 and 2 show two AF segments from patient 3 while panels 3 and 4 represent AF segments from patients 5 and 6 respectively.

\subsubsection{Atrial fibrillatory signals on the body surface}

As it can be observed in Figure 6.4, in some cases similar signals can be found in any position on the body surface in terms of their amplitude, rate and number of deflections (Figure 6.4.1). In other cases, there is no clear relationship between the signals observed in different points on the body surface (see Figure 6.4.2$6.4 .4)$. 


\subsubsection{Surface activation patterns during AF}

Maps were generated for all the segments and visually inspected. Single or multiple simultaneous wavefronts, wave breakages and disorganized electrical activity could be observed. Figure 6.5 shows some selected wavefront propagation maps in which some representative wavefront patterns can be observed.

In panel 1a, a succession of wavefronts from patient 3 is observed. A single wavefront can be observed in both panels $1 \mathrm{a}$ and $1 \mathrm{~b}$. The time interval between segments a and b was not displayed, as it represents the repolarization wavefront. In panel 1a, wavefronts are mostly parallel to each other, with activation starting in the back (this would change if a different time lag was selected) and spreading towards the front. Panel $1 \mathrm{~b}$ shows the next succession of wavefronts. The direction of propagation in panel $1 b$ of the second wavefront does not differ significantly from panel 1a.

Panel 2 shows a segment covering at least one cycle length of the fibrillatory waves, which has been divided into two consecutive intervals displayed in panels $2 \mathrm{a}$ and $2 \mathrm{~b}$. As can be observed in panel $2 \mathrm{a}$, the wavefront exhibits a rotation around $270^{\circ}$. In panel $2 \mathrm{~b}$, the wavefront propagation completes the $360^{\circ}$ circle and starts a new cycle. The same propagation pattern was observed in the consecutive intervals (2.5 more cycles).

Panel 3 shows two consecutive depolarization waves from patient 5 . Depolarization wave shown in panel $3 \mathrm{a}$ is first shown in the leftmost part on bottom of the grid and spreads towards the center and top until it gets blocked. A second depolarization wave starts in the uppermost part on the right of the grid and propagates towards the bottom and the left until it gets blocked in roughly the same position where the previous wave arrived (see panel $3 \mathrm{~b}$ ). It can be observed that one wavefront is blocked by the previous propagation contributing to a chaotic propagation.

Panels $4 \mathrm{a}$ and $4 \mathrm{~b}$ show depolarization waves in patient 6 . Multiple waves arise at the front part at the same time, propagate, join or break in an uncoordinated fashion while a more coordinated pattern is observed in the back. There is no clear propagation pattern, and even consecutive segments vary to a great extent.

\subsubsection{Surface activation patterns during AF and similarity among AF signals on the body surface}

Similarity among atrial fibrillatory signals observable on the body surface can be understood by analyzing the surface activation patterns that correspond to each segment. A very regular pattern can be observed in Figure 6.4.1 which corresponds to a single wavefront propagating across the whole surface (see Figure 6.5.1). In this case, all atrial fibrillatory signals are similar in terms of amplitude, rate, number of zero-crossings and wave deflections and study of a 
1

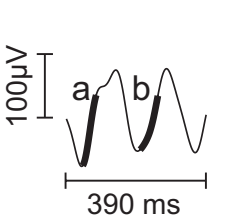

2

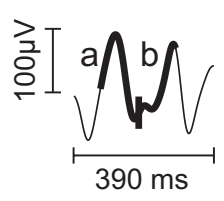

3

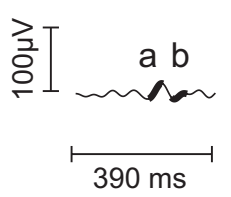

4

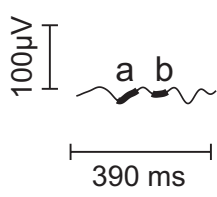

$1 a$

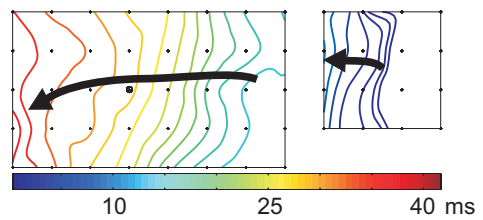

$2 a$

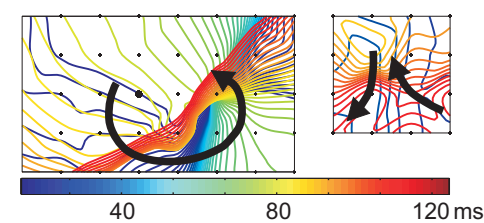

$3 a$

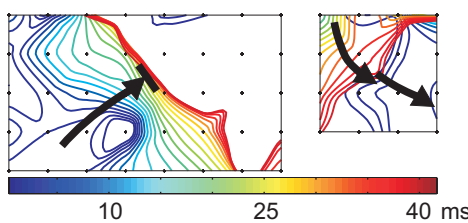

$4 a$

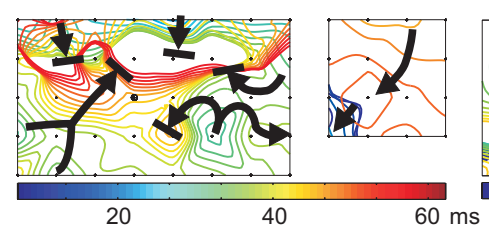

$1 b$

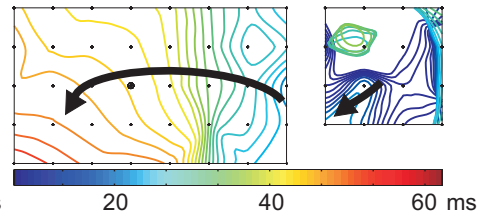

$2 b$

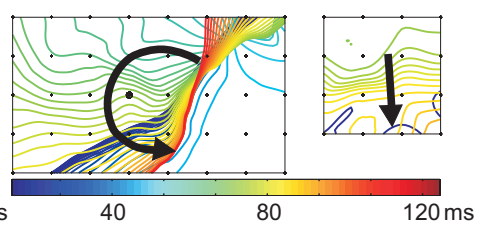

$3 b$

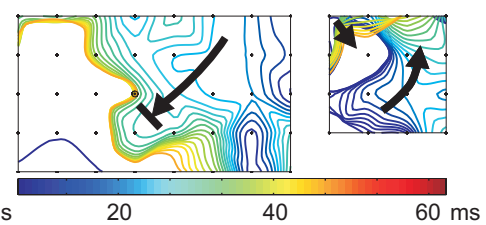

$4 \mathrm{~b}$

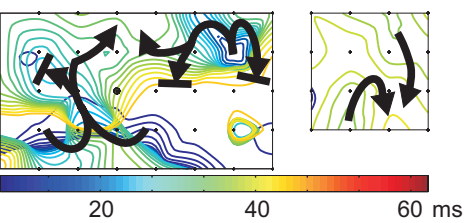

Figure 6.5: Wavefront propagation maps of the four selected AF segments depicted in Figure 6.3. On the left, tracings corresponding to V1 for the segments chosen. Panels a and b correspond to wavefront propagation maps of two intervals of the same segment in a color scale. The segments represented in panels $\mathrm{a}$ and $\mathrm{b}$ are denoted by heavier lines in the tracings. On the left in each panel, maps corresponding to the front part of the thorax. On the right, maps corresponding to the back. Each electrode position is labelled with a '+' sign. V1 is represented by a circle. Wavefront propagation lines are drawn every $2 \mathrm{~ms}$. According to the color scale, wavefronts appearing first are drawn in blue, while wavefronts appearing last are drawn in red. Notice that the color scale is different in each figure according to the length of the interval selected. Arrows indicate the direction of propagation of each wavefront. 
single lead (e.g. $V_{1}$ ) may be representative of the electrical activity observed in the whole body surface.

In figure 6.5.2 a rotating wavefront is depicted. In this case, a fairly regular pattern can be appreciated in most leads in Figure 6.4.2, except for the locations close to the rotation axis (e.g. for the lead highlighted in a dashed line). As observed, the leads around the rotation axis present lower amplitude and more deflections.

A similar observation can be extracted from Figure 6.4.3, where a regular pattern of the atrial fibrillatory wave is observed both in the upper right and bottom left corners, corresponding to activations propagating across these sites. On the contrary, sites close to diagonal where the blockage of the wavefront propagation occurs (see Figure 6.5.3) show different morphology and frequency. Lead V1 stands among the leads belonging to this area, which differ in amplitude, number of deflections or rate from signals recorded from other parts of the body.

Finally, in Figure 6.4.4, the morphology of fibrillatory waves is different in every area of the body surface, except for the back, where the pattern is more regular. Wavefront propagation maps confirm a very irregular pattern, with multiple simultaneous wavefronts on the front part of the torso.

\subsubsection{Short-term reproducibility of dominant activation patterns}

Wavefront propagation maps were classified by visual inspection. Maps in figures $6.5 .1 \mathrm{a}, 6.5 .1 \mathrm{~b}, 6.5 .2 \mathrm{a}$ and $6.5 .2 \mathrm{~b}$ were classified as type I because single wavefronts appear without splitting or blocking. Notice that the direction of propagation in different segments from one patient may differ to a greater extent. However, these changes may occur gradually and are not visible in a single segment. Maps 3a and 3b were classified as type II because blockage of single wavefronts is observed. Maps $4 \mathrm{a}$ and $4 \mathrm{~b}$ were classified as type III because multiple simultaneous wavefronts are observed.

Table 6.3 shows the classification of all maps of all patients. As it can inferred from Table 6.3, wavefront propagation patterns were repeatable and similar results were observed when analyzing different time intervals except for patients 6 and 11, resulting in a correlation value of $0.95(\mathrm{p}<0.01)$. There was no statistical difference neither in the number of segments studied nor in their mean length in type 1 patients and compared to type 3 patients: $86 \pm 78$ vs. $48 \pm 47$ segments $(\mathrm{p}=\mathrm{ns})$ and $512 \pm 109$ vs. $497 \pm 85 \mathrm{~ms}(\mathrm{p}=\mathrm{ns})$. 


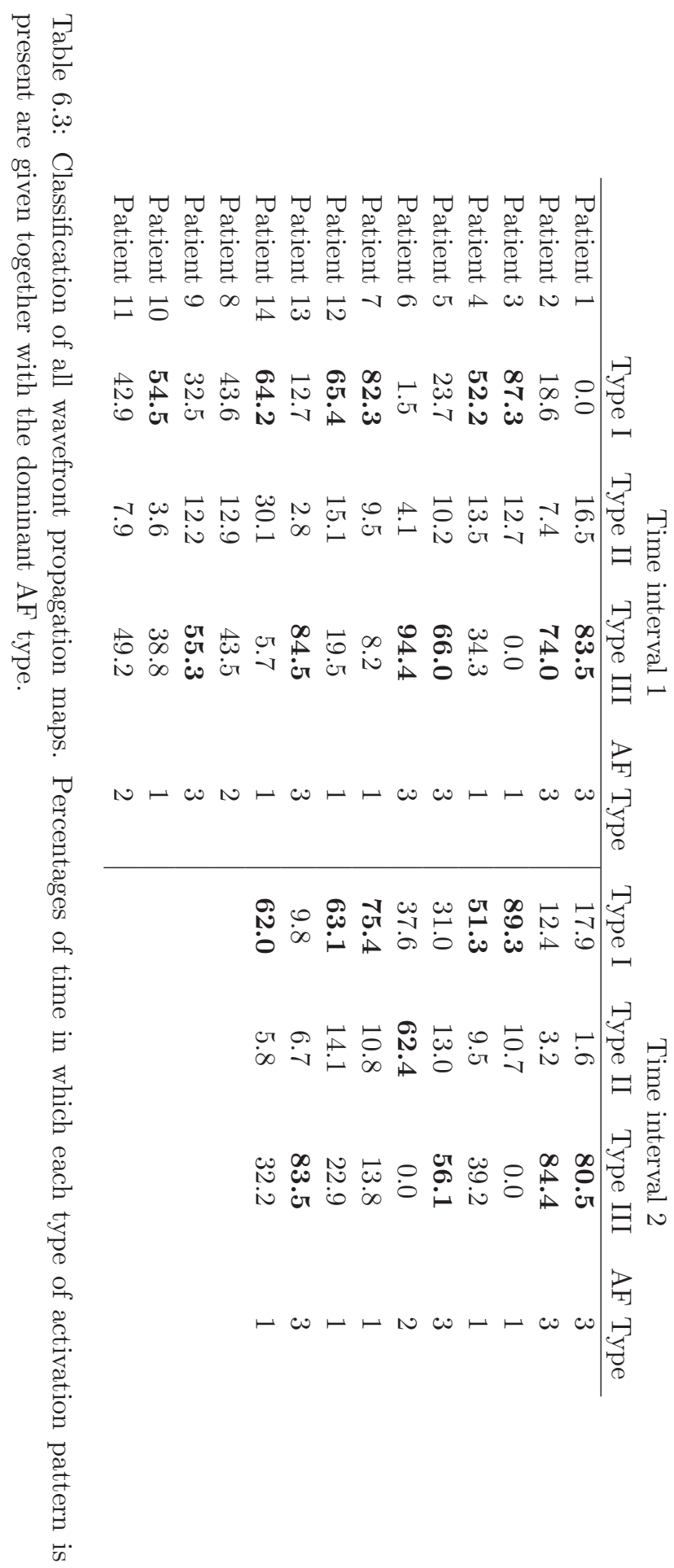




\subsection{Discussion}

\subsubsection{Main findings}

This study is the first to apply a custom-made ECG lead system to perform non-invasive high-density surface mapping during AF. Despite the low signal-tonoise ratio of AF signals, several activation patterns with different organization degrees could be identified by using this mapping system and signal processing described. Interestingly, there was inter-individual variability of the dominant activation pattern, which was highly reproducible.

\subsubsection{Comparison with invasive mapping data}

Our observations are consistent with the circuits observed in invasive studies or optical mapping experiments in which rotors, spiral waves, wave splitting and wave breaking have been observed $[2,33,3]$. Despite the smoothing effect of the body organs plus the addition of noise, AF signals recorded on the body surface allow the construction of wavefront propagation maps in which either a rotating wavefront, or multiple wavefronts can be observed.

Three different waveform propagation patterns could be identified similar to those observed by Konings et al. [71], related to different degrees of complexity in atrial electrical activation. Type I propagation maps correspond to activation of the atria due to a single wavefront. Type II propagation maps correspond to activation of the atria due to a limited number of wavefronts that do not propagate across the entire atrial mass, suffering breakage and splitting. Type III propagation maps correspond to a more complex pattern in which multiple wavefronts appear simultaneously. In our study group, $43 \%$ of patients showed mostly type I activation while $43 \%$ of patients showed mostly type III activation during minutes 1-2 of the recording, while $14 \%$ patients presented mixed type I and type III patterns. Our percentages differ from those reported by Konings (40\% type I, $32 \%$ type II, $28 \%$ type III in 25 patients with induced AF) but this difference can be attributed to the clinical characteristics of their patients as all their patients presented apparently normal atria and normal spread of activation during sinus rhythm and pacing, which may not be representative of the characteristics of patients with chronic AF in which the atrial remodelling may be present. A low percentage of type II segments were identified in our database compared to those reported by Konings. This can be attributed to our larger sampling region that allows the observation of a higher number of simultaneous wavefronts.

Apart from illustrative purposes, identification of the type of fibrillation may be useful for quantifying the degree of organization of AF in each patient. Everett et al. [32] demonstrated that previous knowledge of the degree of organization in the atria by analyzing the frequency spectrum of atrial electrograms is useful for optimizing shock efficacy during electrical 
cardioversion. Our method could help to obtain similar information in a non-invasive manner and thus reducing risks and costs.

\subsubsection{Comparison with non-invasive studies}

Body Surface Mapping of atrial events was first described by Sippensgroenewegen at al. in characterizing $\mathrm{P}$ waves during ectopic atrial activation [118] and during atrial flutter [115]. However, this is the first time a BSPM system has been used to represent body surface maps during AF and different activation patterns have been described.

Analysis of the frequency content of AF signals on the body surface has been shown to be correlated with the analysis of the frequency content of electrograms $[108,66]$. This technique seems to be useful for monitoring and predicting response to therapy, such as antiarrhythmic drugs or cardioversion [11]. However, the study of standard ECG signals during AF has the limitation that only a few signals are available and most of them face the left ventricle. In addition, fibrillatory frequency is just one parameter that describes part of the fibrillatoy process. Among the leads available, V1 is the most used lead for characterizing AF signals because its proximity to the atria. However, as it can be inferred from our study, the use of a limited number of leads, or the use of V1 alone, may or may not be representative of the voltages that can be recorded from the whole body surface. In case a single activation wavefront is present, a single lead (e.g. $V_{1}$ ) may be representative of the overall activation of the atria. However, this is not true when $V_{1}$ faces the rotating axis of the wavefront. If there is a single activation wavefront that does not propagate across the whole atria or multiple wavefronts coexist, it cannot be stated that the signal recorded in lead $V_{1}$ is representative for the overall atrial activation and additional information extracted from other sites is required.

\subsubsection{Limitations}

Correspondence of our findings with the actual mechanisms of AF in each patient has not been demonstrated because of the lack of simultaneous invasive recordings. Wavefront propagation maps represent a simplification of the electrical field generated by the electrical activity of the entire atrial myocardium projected in two dimensional views from the anterior and posterior sides of the thorax. Although activity in the right atrium may be represented preferentially on the anterior torso and the left atrium on the posterior torso the same way than $V_{1}$ represents mostly right atrial activity and esophageal recordings reflect mostly left atrial activity, further studies including simultaneous intracardiac signals and body surface maps will help in clarifying the potential of this non-invasive technique for the characterization of activation patterns in the atria. It needs to be emphasized, however, that the current technology is not capable of but also not intended to describe local 
atrial activity such as spiral waves or local micro-reentry. It may be viewed as another attempt to non-invasively subdivide AF patterns that is in analogy with fibrillatory rate of ECG lead V1. This measure is also a rather general parameter of electrical atrial activity and not intended for describing fibrillatory rates of certain atrial areas.

As a feasibility study, no analysis has been performed in relation with outcome parameters, e.g. drug effects or recurrence after cardioversion. The clinical relevance of the existence of different activation patterns as observable from the body surface needs to be assessed in future studies.

We used 56 electrodes in order to construct our maps. Although this number is higher than the number of electrodes used in clinical practice, we may not be capturing all information related with the complex dynamics of AF signals and thus our spatial resolution is limited. BSPM maps during AF need to be interpreted carefully because their degree of accuracy may be dependent on the electrical organization degree in the atria. A single wavefront will most likely result in a single wavefront in BSPM maps and its direction could be obtained. In our classification of patients, the direction of each type I and type II wavefronts can be assessed from the body surface. When multiple wavefronts appear simultaneously we may not be able to determine the number of wavefronts and/or their direction. However, we may be able to determine that multiple simultaneous wavefronts appear. The spatial resolution of our maps could be improved by the use of a higher number of electrodes, although the smoothing effect of the inner organs sets a limit in the spatial resolution achievable.

Finally, this study was conducted in a consecutive, but small series of patients with heterogeneous medications and underlying heart diseases

\subsection{Conclusions}

Using electrocardiographic body surface mapping during $\mathrm{AF}$, different activations patterns could be observed with a non-invasive procedure. Inter-individual activation differences can be observed while showing an excellent short-term reproducibility. 



\section{Chapter 7}

\section{Discussion and conclusion}

7.1 Discussion

\subsection{Conclusion}

7.3 Guides for future work

In this chapter a global conclusion of the results given in this dissertation is presented. This chapter has been structured in three sections. First, the main findings of this thesis are summarized and previous works relevant to this topic are discussed in light of our results. Limitations of the methods presented in the previous sections are highlighted. Secondly, the main conclusions of this work are explicitly presented and it is summarized how they satisfy the objectives previously established. Finally, a third section is dedicated to future research lines.

\subsection{Discussion}

Mechanisms sustaining AF are poorly understood. This has motivated the efforts of many research groups that have focused their attention in providing new insights into the understanding of this arrythmia. The Bioengineering group belonging to ITACA, at Universidad Politecnica de Valencia has devoted its last years to this field with a number of research projects, publications and international collaborations. The contribution of this thesis lies in the study of the atrial fibrillatory process from a new perspective, released from the limitations imposed by the dipolar model of myocardial activity. 


\subsubsection{Main findings}

Extraction of spatial characteristics from the surface ECG can be accomplished in the case of sinus rhythm by deriving orthogonal leads from the surface ECG. This derivation is usually performed by making use of transform matrices that optimize for ventricular activity (i.e. Dower's inverse transform). In order to extract spatial indicators for atrial activity during sinus rhythm, a transform matrix which optimizes for atrial activity offers a higher degree of accuracy than a ventricular-based transform matrix. This result confirms our hypothesis that the difference in location of the atria with respect to the ventricles has a nonnegligible effect on the derivation of orthogonal indicators from the standard ECG.

In case of AF, spatial indicators cannot be derived with accuracy from the surface ECG neither with a ventricular-based transform, nor with an atrial based transform. Observation of multiple simultaneous potential maxima and minima on body surface maps confirms the lack of dipolarity of AF signals. This lack of dipolarity of AF signals appears as the main cause of the inaccuracy of the standard ECG for the determination of spatial parameters during AF and not the difference in location of the atria with respect to the ventricles.

Accurate extraction of spatial parameters can be accomplished by obtaining the heart potentials on the entire body surface. The standard electrocardiogram, again, has shown to offer a poor performance in capturing surface potentials during AF although its performance is satisfactory for normal QRS complexes and $\mathrm{P}$ waves. The number and location of the exploring electrodes optimized for capturing heart potentials on the entire body surface during AF has been evaluated in this thesis. First, we evaluated two different methods proposed in the literature for the determination of the number and location of electrodes in optimum lead sets. Although the performance of both algorithms is similar for a high number of electrodes, the performance of the algorithm proposed by Lux [88] offers the best performance for limited lead sets. In the case of AF, the number of electrodes needed is of the order of 23 to have an accuracy similar to the accuracy of the standard ECG on the QRS complex (25\% error). In fact, the location of electrodes may not be a crucial issue for BSPM systems once a certain number of leads has been reached and if the electrodes cover the entire body surface. The number of exploring electrodes, however, is crucial for obtaining a desired accuracy. In the case of AF, a repositioning of the eight standard ECG electrodes improves significantly its accuracy although a real improvement in accuracy is only obtained by increasing the number of electrodes.

The relevance of our results regarding the reconstruction of body surface potentials during $\mathrm{AF}$ and the number and location of exploring electrodes needed for achieving this objective is only justified by the clinical relevance of BSPM maps during AF. We have demonstrated that meaningful BSPM maps can be constructed during AF despite the low signal-to-noise conditions of AF signals. We have been able to observe different activation patterns during AF 
including a single rotating wavefront, single wavefronts that do not propagate across the entire surface or multiple simultaneous wavefronts. These observations were repeatable over time confirming that these propagation maps are not entirely random and may convey some clinical information of the patient.

\subsubsection{Comparison with previous studies}

During the last years, many studies have focused in the extraction of organization measurements of atrial fibrillatory signals from the surface ECG. Spectral analysis of AF signals is related to the temporal organization of AF and in fact the dominant frequency in the spectrum is related to the atrial cycle length [108]. Also, study of the atrial vectorcardiogram has the potential use of providing spatial organization indexes that cannot be extracted by analyzing individual ECG leads. However, most of these studies present limitations that are consequence of the assumption of a dipolar model of the myocardial activation that are not valid for fibrillating atria.

Early BSPM studies demonstrated the existence of a non-dipolar content of the vectorcardiogram that was reflected in the occasional observation of more than three potential extremes on body surface maps [126]. However, the nondipolar content of the QRS complex in healthy individuals was considered to be insignificant [43] and it was accepted that the recording of more than three orthogonal leads was unnecessary. Departing from this assumption, the VCG and the standard ECG contain all relevant diagnostic information displayed in space and time domains respectively. In fact, in chapter 5 we arrive to similar conclusions to those reported in the literature, finding the standard ECG as a good estimate for QRS complexes and P waves.

Conversely, this assumption of dipolarity of the electrical activation is inaccurate for $\mathrm{AF}$ because the observation of multiple potential extremes on body surface maps is not occasional and residual. Consequently, the VCG and the standard ECG may not convey all relevant diagnostic information. Previous studies have already reported the inaccuracy of the standard ECG for the study of AF and proposed a repositioning of the ECG leads moving some electrodes to positions near the atria $[66,67]$. From our results, repositioning of the exploring electrodes improves to some extent the amount of information recorded but does not reach an acceptable accuracy unless the number of exploring electrodes is increased.

The use of a limited number of leads for the study of AF is suboptimal, but in any case it contributes to some knowledge of the state of the atria in each patient without an additional cost. In fact, spectral analysis of lead $V_{1}$ can predict to some extent the success of cardioversion [8] and the effect of certain drugs [10]. However, the use of leads derived from a small subset of leads is completely inaccurate for AF signals. The lack of dipolarity of AF signals has been underestimated in the literature and spatial indicators of AF organization 
obtained from derived VCGs have been reported $[102,106]$. Spatial parameters obtained from orthogonal leads derived from the standard ECG are not related to spatial parameters obtained from non-derived orthogonal leads according to our results presented in chapter 4. More surprisingly, these studies claimed the validity of the analysis of planes of best fit of AF loops without reporting if AF loops fit to a plane. From our results, AF loops do not fit to a plane and thus it is difficult to understand how this analysis can report meaningful information.

Instead of deriving orthogonal leads from the surface ECG, spatial parameters may be obtained by directly recording them, keeping in mind that their amount of information is limited. VCG leads can be obtained with Frank's lead system with the limitation that the electrical origin is positioned in the ventricles -not in the atria- and thus Frank's lead system is not orthogonal. Instead, a VCG optimized for atrial activity can be employed [131].

As previously stated, a lead system optimized for studying atrial activity should employ more electrodes than the standard ECG and more electrodes than for studying a QRS complex with the same degree of accuracy. According to our results presented in chapter 5, 23 leads are needed in order to achieve the same degree of accuracy than 8 leads for the QRS complex. The higher degree of complexity of AF signals is in consonance with previous studies from Hoekema [61] and Ihara [67] who reported that there are 8 independent components in the QRS complex and around 60 for AF signals.

With a sufficient number of leads, body surface potential maps during AF can be constructed. These maps contain most information observable on the body surface and thus spatial organization parameters can be obtained. BSPM maps allow the observation of different degrees of organization that are compatible to those reported by Konings [71] in epicardial mapping studies: a single wavefront, wavefronts that split or break and multiple simultaneous wavefronts. Experiments performed by Konings did not report the possibility of a mother rotor sustaining AF as reported by the group of Jalife [68], but this can be related to the narrow spatial field of view of her multiple electrode. A narrow field of view may prevent the observation of the rotation axis that could be determined in optical mapping experiments in which the electrical activity of entire heart is observed. Our results concerning rotating wavefronts are compatible with those reported by the group of Jalife, although experimental evidence relating surface maps to endocardial or epicardial recordings is needed. In some patients single wavefronts could not be observed, a finding that is compatible both with fibrillatory conduction originated in a mother rotor [69] or with a totally disorganized atrial activity consequence of either electrical or structural remodeling [1].

The advantage of BSPM maps over aforementioned mapping systems is that they are recorded non-invasively and they offer a wide field of view of atrial electrical activation. The limitations of BSPM maps over invasive mapping 
systems is that surface potential signals contain both information from the atria and the ventricles and that spatial resolution is lower due to the smoothing effect of the inner organs.

\subsubsection{Limitations}

The main limitation of this work is that the number of patients studied is not sufficient for extrapolating our results to a general population. However, the methods and techniques described in all chapters of this thesis may be applicable to larger populations in order to confirm our observations.

Another limitation of this study is the lack of a satisfactory method for extracting atrial activity from the surface ECG free from ventricular content. Due to this drawback we had to concentrate our analysis in the study of ECG segments in between ventricular beats. This has prevented us to study repeatability over consecutive beats and our temporal organization analysis had a poor time resolution.

\subsection{Conclusion}

In this thesis we have accomplished all the objectives that were established. A brief response to each objective stated in section 1 is detailed in this section.

\section{Objective:}

- Evaluate the possibility of extracting spatial parameters from the standard $E C G$

Response: Orthogonal leads, and thus spatial indicators, cannot be derived with accuracy from the standard ECG during AF neither with a ventricle-based transform nor with an atrial-based transform. This lack of accuracy is related to the non-dipolarity of the atrial electrical activation to a greater extent than the different location of the atria with respect to the ventricles.

\section{Objective:}

- Determine the number and location of exploring ECG electrodes most appropriate for the recording of atrial electrical activity during AF

Response: The number of exploring electrodes should be as high as possible in order to capture most electrical information in non-dipolar AF signals. If a limited lead set needs to be chosen, lead systems covering the entire body surface including right anterior, left anterior and posterior electrodes are preferable to the standard ECG. Accuracy of limited lead systems is, however, limited by the number of exploring electrodes and 
will not reach the same accuracy than the standard ECG for the QRS complex unless more than 23 leads are recorded.

\section{Objective:}

- Extract spatial organization patterns from BSPM recordings during AF

Response: Different activation patterns can be observed by analyzing body surface potential maps during AF. These activation patterns reflect different degrees of spatial organization that are repeatable over time.

\subsection{Guides for future work}

This thesis opens a new research line for researchers interested in a better understanding of the mechanisms of $\mathrm{AF}$ and in the disposal of new procedures for the selection of the most suitable therapies for AF in each patient. In this section we will outline some future research lines derived from the results of this thesis.

\subsubsection{Spatiotemporal quantification of atrial organization}

The methodology described in the present thesis for the analysis of spatial properties of the activation patterns during $\mathrm{AF}$ is restricted to time intervals in the ECG without ventricular content and thus many atrial waves cannot be evaluated. Development of a new method for cancellation of ventricular activity in BSPM recordings would allow a continuous mapping and a better understanding of the dynamics of atrial fibrillatory waves. This cancellation method could be based in spatiotemporal alignment for average beat subtraction and exploit the large number of leads recorded and their spatial relationship to improve its performance.

Most signal processing techniques described regarding signal conditioning and detection of TQ intervals can be applied with a minimum intervention of a technician for reviewing detections. Quantification of maps was performed by visual inspection but automatic quantification methods could be implemented. These quantification methods could automatically detect the number of simultaneous wavefronts and their direction providing new markers based both in the number of wavefronts and the repeatability of their directions.

Analysis of BSPM recordings during AF could be extended to the study of both spatial and temporal variability and include spectral analysis of all leads recorded. It would be of interest to compare organization indexes obtained by spatial analysis of maps, temporal variability of wavefronts and spectral analysis of multiple leads with classical organization measurements such as the dominant frequency in $V_{1}$ or spatial parameters derived from recorded VCGs. According to our observations regarding the variability of simultaneous ECG recordings 
obtained at different locations on the body surface, we expect that the study of more leads apart from $V_{1}$ or $X, Y$ and $Z$ leads will provide a more detailed description of the fibrillatory process in each patient. However, a more rigorous analysis would help clarifying the relevance of both approaches.

\subsubsection{Clinical applications}

Clinical relevance of the methods presented in this thesis should be assessed by comparison with either intracardiac or epicardial mapping recordings. This comparison would help in determining whether organization measurements obtained from surface maps are similar to those obtained from intracardiac recordings, with less prominent ventricular interference, higher signal to noise ratios and without the smoothing effect of the lungs, bones and other inner organs.

Rotating wavefronts could be observed in some of our patients. It would be of interest to study whether these rotors are the mechanism sustaining AF in those patients and thus if BSPM recordings allow to determine the underlaying mechanism of AF in these group of patients. More importantly, the possible determination of the existence of ectopic focus near the pulmonary veins by studying the wavefront propagation patterns and organization indexes in the anterior and posterior faces of the torso would be of a great clinical relevance. Findings regarding the mechanisms sustaining AF in each patient could be used for predicting the success of different therapeutical strategies. Determination of a pulmonary vein origin should be related to a greater success of pulmonary vein isolation therapies while a very low organization degree would point to pharmacologic therapy as the most reasonable alternative. Additionally, organization measurements obtained with the methods described in this thesis could also be of interest for determining the effect on atrial organization of some antiarrhythmic drugs whose mechanism of action is not completely understood.

Finally, techniques and methods developed in this thesis could be applied for the analysis of other atrial arrhythmias such as atrial flutter. Determination of the propagation pattern of AFL may help in planning the ablation strategy in cases of atypical flutter in which the uncertainty on the location of the entrainment circuit needs to be determined during the intervention with electroanatomical mapping systems.

\subsubsection{Transfer of technology}

The methods needed for measuring spatial organization during AF from BSPM recordings have been successfully implemented and tested. Application of these methods would be of interest for researchers, clinicians and manufacturers of medical monitoring devices. 
Multiple research groups around the world are working in clarifying the mechanisms underlaying AF, which are not completely understood. Many of these research groups, some of which are already collaborating with us, will be interested in exploring the capabilities of the methods developed for this thesis. Also, many clinicians might be interested in applying these methods for the diagnosis of their patients and selection of the most suitable therapy in each case.

In case future studies provide clinical evidence of the benefits of this technology, transfer to manufacturer companies will be a matter of time. New monitoring devices could record more surface leads, compute maps and quantify organization helping clinicians in their diagnosis.

High acquisition times and elevated costs per recording have prevented BSPM systems to be introduced into clinical practice despite their diagnostic capabilities which are specially relevant in life threatening conditions such as AMI. Methods described in this thesis allow the recording and analysis of BSPM signals in the same time needed for a standard ECG. Transfer of technology of both the electrode supporting system and signal processing methods described in this thesis could extend the use of BSPM to other clinical applications beyond the diagnosis of atrial fibrillation. 


\section{Chapter 8}

\section{Contributions}

As it occurs in most research works, this dissertation can not be regarded as an isolated item. On the contrary, it has been developed within the framework of current research projects, and having established close contacts with other research groups and institutions. In addition, the most important advances achieved in this thesis have been divulged in top level conferences and journals with high impact factor indices.

\subsection{Publications}

This thesis is supported by the publication of part of its content in two articles already published in an international journal, two articles in review process of two different international journals and one paper already accepted for publication in a high impact journal, all of them listed in the Scientific Citation Index.

\subsubsection{Main contributions of this thesis}

- Derivation of orthogonal leads from the 12-lead electrocardiogram. Performance of an atrial-based transform for the derivation of $\mathbf{P}$ loops [58], which appeared in Journal of Electrocardiology (Impact Factor: 1.069, Ranking 55/74 in category Cardiac and Cardiovascular systems). This publication evaluated the performance of classical methods for deriving orthogonal leads from the standard ECG on atrial components during sinus rhythm and proposes a new transform method optimized for atrial activity.

- Limitations of Dower's inverse transform for the study of atrial loops during atrial fibrillation [51], which is under revision process in Pacing and Clinical Electrophysiology (Impact Factor: 1.561, Ranking 
22/44 in category Biomedical Engineering). This manuscript evaluated the performance of Dower's inverse transform and for the derivation of orthogonal leads during atrial fibrillation. Other P-wave optimized and QRS-optimized transforms were also evaluated.

- Evaluation of lead selection methods for optimal reconstruction of body surface potentials [58], which appeared in Journal of Electrocardiology. This publication evaluates two different methods proposed in the literature for the determination of optimum lead sets departing from BSPM recordings.

- How many leads are necessary for a reliable reconstruction of surface potentials during atrial fibrillation? [47], which is under revision in IEEE Transactions on Information Technology in Biomedicine (Impact Factor: 1.436, ranking 27/92 in category Computer Science, Information Systems). This paper evaluated the minimum number of electrodes which allow an accurate reconstruction of body surface potentials during atrial fibrillation.

- Non-invasive mapping of atrial fibrillation [52], which has been accepted for publication in Journal of Cardiovascular Electrophysiology (Impact Factor: 3.475, Ranking 15/74 in category Cardiac and Cardiovascular systems). This publication describes the techniques used for constructing body surface potential maps during atrial fibrillation and the propagation patterns that we have observed in humans.

In addition, the main contributions of this thesis have been spread in diverse conferences, with a high acceptance $[57,50,55,17,54,53]$.

Regarding awards, it must be pointed out that the work presented at the conference Biosignal'08 in Brno, Czech Republic, was awarded as the best student paper.

\subsubsection{Contributions related to this thesis}

- Analysis of the extension of Q-waves after infarction with body surface map: Relationship with infarct size [6], which appeared in International Journal of Cardiology (Impact Factor: 2.878, Ranking 22/74 in category Cardiac and Cardiovascular systems). This publication evaluated the clinical relevance of pathological Q waves in the surface ECG and how their extension on the body surface relates to infarct size.

- Bidimensional histogram profiles of RR intervals. A novel noninvasive method for the evaluation of preferential AV nodal conduction during atrial fibrillation [19], which is In Press in IEEE Transactions on Biomedical Engineering (Impact Factor: 1.622, Ranking 19/44 in category Biomedical Engineering). In this article, a new 
method for the evaluation of the ventricular response during atrial fibrillation is proposed.

- Improving The Diagnosis Of Bundle Branch Block By The Analysis Of Body Surface Potential Maps [24], which is under revision in Journal of Electrocardiology. This publication describes the possible use of BSPM for an automatic diagnosis of bundle branch block.

Part of the work accomplished in advance to this thesis regarding the electrode holding system resulted in the publication of a Spanish patent [56].

Finally, research works related to this thesis have been presented in Spanish and international conferences in the fields of biomedical engineering, signal processing and cardiology, with a total of 29 contributions.

\subsection{Framework of the Dissertation}

This dissertation has been carried out within the Bioengineering group at the Institute for the Applications of Advanced Information and Communication Technologies (Bio-ITACA) at Universitat Politècnica de València. The researchers in the Bioengineering area, leaded by Dr. José Millet, have contrasted expertise in the analysis of cardiac arrhythmias from the ECG, such as ventricular fibrillation, ventricular tachycardia, etc. Research at BeT has been devoted during the last years to the analysis of atrial fibrillatory signals, and particulary to the extraction of AF components from the surface ECG. More recently, the research at Bio-ITACA has focused in the study of BSPM recordings as an improvement in the diagnosis of several diseases, being the only Spanish research group in possession of a BSPM system.

\subsubsection{Research projects}

This work has been developed within the framework of several research projects. The interest and relevance of the research activity carried out in the Bio-ITACA group has been acknowledged by public administrations, which have contributed with important economical support to the following research projects:

- Ministerio de Educación y Ciencia.

Exploración de nuevas técnicas de procesado y analisis de la fibrilación auricular. Aportación de los sistemas multi-derivación.

From Oct. 15th, 2005 until Oct. 14th, 2008.

Budget: $84.133 €$.

- Ministerio de Ciencia y Tecnología.

Extracción y caracterización de la actividad auricular mediante Blind Source Separation como ayuda al diagnóstico clínico. 
From Dec. 1st, 2002 until Dec. 1st, 2005.

Budget: $141.890 €$.

- UPV. Vicerrectorado de Investigación, Desarrollo e Innovación.

Exploración de nuevas técnicas de procesado y analisis de la fibrilación auricular. Aportación de los sistemas multi-derivación.

From Dec. 21st, 2006 until Dec. 21st, 2007.

Budget: $13.200 €$.

- Generalitat Valenciana. Conselleria de Empresa, Universitat y Ciencia

Clasificación y caracterización de taquiarritmias auriculares mediante distribuciones tiempo-frecuencia

From Jan. 1st, 2004 until Jan. 1st, 2006.

Budget: $20.450 €$.

- UPV. Vicerrectorado de Investigación, Desarrollo e Innovación.

Sistemas de Adquisición, representación y análisis de señales BSPM.

From Dec. 10th, 2004 until Dec. 10th, 2006.

Budget: $7.250 €$.

- Ministerio de Educación y Ciencia.

Efectos electrofisiológicos del ejercicio físico sobre la excitabilidad miocárdica.

From Oct. 1st, 2006 until Oct. 1st, 2009.

Budget: $7.028 €$.

- Instituto de Salud Carlos III.

Valoración de la cardiopatía isquémica mediante métodos avanzados de imagen.

From Dec. 30th, 2004 until Dec. 30th, 2007.

Budget: $21.160 €$.

- Generalitat Valenciana. Conselleria de Empresa, Universitat y Ciencia Ayuda complementaria $G V$ al proyecto: valoración de la cardiopatía isquémica

From Jan. 1st, 2006 until Jan. 1st, 2007.

Budget: $5.600 €$. 


\subsubsection{International research stays}

During the development of this thesis MS Guillem was awarded with the degree of Master's in Biomedical Engineering in 2006 from Northwestern University in Evanston, USA thanks to a Fulbright fellowship. During the academic year 2005-2006 her research activity was carried out within the group leaded by Prof. Alan Sahakian and Dr. Steven Swiryn, focusing in the analysis of the electrocardiogram in atrial arrhythmias.

Later, a 3 months research stay was performed within the group leaded by Dr. Andreas Bollmann at Leipzig Heart Center, Leipzig University (Germany). In this stage, it was started a joint project for the analysis of BSPM recordings during AF.

In addition, a stay of 1 month and two weeks was also performed within the group leaded by Dr. Ramon Brugada, at Montreal Heart Institute in Canada. In this stage, it was started a research project for the contributions of BSPM to the understanding of electrocardiographic patterns present in Brugada syndrome and during cardiac resynchronization therapy.

\subsubsection{Collaborations}

Within the general framework of this thesis, the contacts with the following research groups and institutions have been established and reinforced:

Hospital Clínico Universitario de Valencia, Spain. The cardiologists at the Servicio de Hemodinámica, leaded by Dr. Chorro Gascó and Dr. Bodí, have helped us with the acquisition of BSPM recordings of patients with $\mathrm{BBB}$, old $\mathrm{MI}$ and $\mathrm{VH}$ and also with the interpretation of the results of our studies. This collaboration has resulted in several publications and communications [6, 49, 24, 17, 50].

Northwestern University, Evanston, USA During a stay at the group leaded by Pr. Alan Sahakian and Dr. Swiryn a project regarding the accuracy of classical derivation methods for the reconstruction of orthogonal leads from the surface ECG was performed. This collaboration resulted in the publication of one manuscript [58] and one conference communication [57].

University of Madgeburg and Leipzig University, Germany Dr. Andreas Bollmann is currently one of the most active cardiologists in AF research. After carrying out a 3-month stage within his research group, the author is further collaborating with Dr. Bollmann in a joint work whose main objective is to extend the analysis of BSPM maps during AF and its possible use as a predictor of success of cardioversion and ablation therapies. This collaboration has resulted in several publications and collaborations $[52,51,47,55,54,53]$ 
Montreal Heart Institute, Canada After a stay at Montreal Heart Institute under the supervision of Dr. Ramon Brugada, several research projects including the analysis of body surface maps of patients with Brugada syndrome and the potential use of BSPM for the optimization of programming parameters in cardiac resynchronization devices have been started and have resulted in one conference communication [48].

Participation in research networks: The groups in Lund University and Leipzig, together with other research groups from Finland, Norway and Denmark, are collaborating in a research network financially supported by nordic countries. This network is composed of technical and clinical researchers, and the main objective is to improve current knowledge on AF. The Bio-ITACA research group at UPV is also integrated in this research network as an external partner. 


\section{Bibliography}

[1] M. Allessie, J. Ausma, and U. Schotten. Electrical, contractile and structural remodeling during atrial fibrillation. Cardiovascular Research, 54(2):230-246, 2002.

[2] R. Arora, S. Verheule, L. Scott, A. Navarrete, V. Katari, E. Wilson, D. Vaz, and J. E. Olgin. Arrhythmogenic substrate of the pulmonary veins assessed by high-resolution optical mapping. Circulation, 107(13):18161821, Apr 2003.

[3] F. Atienza and J. Jalife. Reentry and atrial fibrillation. Heart Rhythm, 4(3):S13-S16, 2007.

[4] V. Batchvarov, K. Hnatkova, A. Ghuran, J. Poloniecki, A. J. Camm, and M. Malik. Ventricular gradient as a risk factor in survivors of acute myocardial infarction. Pacing and Clinical Electrophysiology, 26(1 Pt 2):373-376, Jan 2003.

[5] D. W. Benson, R. Sterba, J. J. Gallagher, A. Walston, and M. S. Spach. Localization of the site of ventricular preexcitation with body surface maps in patients with Wolf-Parkinson-White syndrome. Circulation, 65(6):1259-1268, June 1982.

[6] V. Bodi, J. Sanchis, M. S. Guillem, J. Nunez, M. P. Lopez-Lereu, C. Gomez, D. Moratal, F. J. Chorro, J. Millet, and A. Llacer. Analysis of the extension of Q-waves after infarction with body surface map: Relationship with infarct size. International Journal of Cardiology, 111(3):399-404, Aug 2006.

[7] A. Bollmann. Quantification of electrical remodeling in human atrial fibrillation. Cardiovascular Research, 47(2):207-209, 2000.

[8] A. Bollmann, D. Husser, M. Stridh, L. Sörnmo, M. Majic, H. U. Klein, and S. B. Olsson. Frequency measures obtained from the surface electrocardiogram in atrial fibrillation research and clinical decisionmaking. Journal of Cardiovascular Electrophysiology, 14(10):S154-S161, Oct 2003. 
[9] A. Bollmann, D. Husser, M. Stridh, L. Sörnmo, M. Majic, H. U. Klein, and S. B. Olsson. Frequency measures obtained from the surface electrocardiogram in atrial fibrillation research and clinical decisionmaking. Journal of Cardiovascular Electrophysiology, 14(10 Suppl):S154S161, Oct 2003

[10] A. Bollmann, N. K. Kanuru, K. K. McTeague, P.F. Walter, D. B. DeLurgio, and J. J. Langberg. Frequency analysis of human atrial fibrillation using the surface electrocardiogram and its response to ibutilide. The American Journal of Cardiology, 81(15):1439-1445, 1998.

[11] A. Bollmann and F. Lombardi. Electrocardiology of atrial fibrillation Current knowledge and future challenges. IEEE Engineering in Medicine and Biology Magazine, 25(6):15-23, Nov 2006.

[12] G.W. Botteron and J.M. Smith. A technique for measurement of the extent of spatial organization of atrial activation during atrial fibrillation in the intact human heart. IEEE Transactions on Biomedical Engineering, 42(6):579-586, Jun 1995.

[13] R. Brugada, T. Tapscott, G. Z. Czernuszewicz, A. J. Marian, A. Iglesias, L. Mont, J. Brugada, J. Girona, A. Domingo, L. L. Bachinski, and R. Roberts. Identification of a genetic locus for familial atrial fibrillation. New England Journal of Medicine, 336(13):905-911, 1997.

[14] H. C. Burger and J. B. van Milaan. Heart-vector and leads. British Heart Journal, 8:157-161, 1946.

[15] G. Calcagnini, F. Censi, A. Michelucci, and P. Bartolini. Descriptors of wavefront propagation - Endocardial mapping of atrial fibrillation with basket catheter. IEEE Engineering in Medicine and Biology Magazine, 25(6):71-78, Nov 2006.

[16] J. Carlson, R. Havmoller, A. Herreros, P. Platonov, R. Johansson, and B. Olsson. Can orthogonal lead indicators of propensity to atrial fibrillation be accurately assessed from the 12-lead ECG? Europace, 7:S39S48, 2005.

[17] F. Castells, M.S. Guillem, A.M. Climent, V. Bodi, F.J. Chorro, and J. Millet. Performance evaluation in the reconstruction of body surface potentials from reduced lead systems. a comparative study of lead selection algorithms. Computers in Cardiology, Durham, EE.UU., 2007.

[18] F. Castells, C. Mora, J. J. Rieta, D. Moratal-Perez, and J. Millet. Estimation of atrial fibrillatory wave from single-lead atrial fibrillation electrocardiograms using principal component analysis concepts. Medical and Biological Engineering and Computing, 5:557-560, 2005. 
[19] A. M. Climent, M. S. Guillem, D. Husser, F. Castells, J. Millet, and A. Bollmann. Bidimensional histogram profiles of RR intervals. A novel noninvasive method for the evaluation of preferential AV nodal conduction during atrial fibrillation. IEEE Transactions on Biomedical Engineering, In Press, 2008.

[20] J.D. Cooksey, M. Dunn, and E. Massie. Clinical vectorcardiography and electrocardiography. Year book medical publishers, Chicago-London, 1977.

[21] I. K. Daskalov and I. I. Christov. Electrocardiogram signal preprocessing for automatic detection of QRS boundaries. Medical Engineering and Physics, 21(1):37-44, 1999.

[22] T. Date, T. Yamane, K. Inada, S. Matsuo, Y. Kanzaki, S. Miyanaga, K. Shibayama, H. Miyazaki, K. Sugimoto, I. Taniguchi, and S. Mochizuki. The effects of pulmonary vein isolation on the morphology of $\mathrm{P}$ waves: the contribution of pulmonary vein muscle excitation to the formation of $\mathrm{P}$ waves. Pacing and Clinical Electrophysiology, 30(1):93-101, Jan 2007.

[23] A. De Simone, G. Stabile, D. F. Vitale, P. Turco, M. Di Stasio, F. Petrazzuoli, M. Gasparini, C. De Matteis, R. Rotunno, and T. Di Napoli. Pretreatment with verapamil in patients with persistent or chronic atrial fibrillation who underwent electrical cardioversion. Journal of the American College of Cardiology, 34(3):810-814, Sept 1999.

[24] V. Donis, M.S. Guillem, A.M. Climent, F. Castells, F.J. Chorro, and J. Millet. Improving the diagnosis of bundle branch block by the analysis of body surface potential maps. Journal of Electrocardiology, Under revision, 2008.

[25] G. E. Dower. A lead synthesizer for the Frank system to simulate the standard 12-lead electrocardiogram. Journal of Electrocardiology, 1(1):101-116, 1968.

[26] G. E. Dower. The ECGD: a derivation of the ECG from VCG leads. Journal of Electrocardiology, 17(2):189-191, 1984.

[27] G. E. Dower and H. B. Machado. XYZ data interpreted by a 12lead computer program using the derived electrocardiogram. Journal of Electrocardiology, 12(3):249-261, Jul 1979.

[28] G. E. Dower, H. B. Machado, and J. A. Osborne. On deriving the electrocardiogram from vectoradiographic leads. Clinical Cardiology, 3(2):87-95, Apr 1980.

[29] L. Edenbrandt and O. Pahlm. Vectorcardiogram synthesized from a 12-lead ECG: superiority of the Inverse Dower Matrix. Journal of Electrocardiology, 21(4):361-367, 1988. 
[30] W. Einthoven. Le telecardiogramme. Archives Internationales de Physiologie, 4:132-64, 1906.

[31] W. Einthoven. Weiteres ber das elektrokardiogram. Pflger Arch. ges. Physiol., 122:517-48, 1908.

[32] T. H. Everett, J. R. Moorman, L. C. Kok, J. G. Akar, and D. E. Haines. Assessment of global atrial fibrillation organization to optimize timing of atrial defibrillation. Circulation, 103(23):2857-2861, Jun 2001.

[33] T. H. Everett and J. E. Olgin. Basic mechanisms of atrial fibrillation. Cardiology Clinics, 22(1):9-20, Feb 2004.

[34] T. H. Everett, E. E. Wilson, S. Verheule, J. M. Guerra, S. Foreman, and J. E. Olgin. Structural atrial remodeling alters the substrate and spatiotemporal organization of atrial fibrillation: a comparison in canine models of structural and electrical atrial remodeling. American Journal of Physiology-Heart and Circulatory Physiology, 291(6):H2911-H2923, Dec 2006.

[35] T.H. Everett, Lai-Chow Kok, R.H. Vaughn, R. Moorman, and D.E. Haines. Frequency domain algorithm for quantifying atrial fibrillation organization to increase defibrillation efficacy. IEEE Transactions on Biomedical Engineering, 48(9):969-978, Sep 2001.

[36] L. Faes and F. Ravelli. A morphology-based approach to the evaluation of atrial fibrillation organization. IEEE Engineering in Medicine and Biology Magazine, 26(4):59-67, July-Aug. 2007.

[37] D. D. Finlay, C. D. Nugent, M. P. Donnelly, R. L. Lux, P. J. McCullagh, and N. D. Black. Selection of optimal recording sites for limited lead body surface potential mapping: a sequential selection based approach. $B M C$. Medical Informatics and Decision Making, 6:9, 2006.

[38] D. D. Finlay, C. D. Nugent, J. A. Kors, Herpen G. van, M. P. Donnelly, P. J. McCullagh, and N. D. Black. Optimizing the 12lead electrocardiogram: a data driven approach to locating alternative recording sites. Journal of Electrocardiolpgy, 40(3):292-299, Jul 2007.

[39] N. C. Flowers, L. G. Horan, and J. C. Johnson. Anterior infarctional changes ossuring during mid and late ventricular activation detectable by surface mapping techniques. Circulation, 54(6):906-913, Dec 1976.

[40] The Research Resource for Complex Physiologic Signals. www.physionet.org. physionet.mit room e25-505a, 2006.

[41] E. Frank. Determination of the electrical center of ventricular depolarization in the human heart. American Heart Journal, 49(5):670692, May 1954. 
[42] E. Frank. The image surface of a homogeneous torso. American Heart Journal, 47(5):757-768, May 1954.

[43] E. Frank. Absolute quantitative comparison of instantaneous QRS equipotentials on a normal subject with dipole potentials on a homogeneous torso model. Circulation Research, 3:243-251, 1955.

[44] E. Frank. An accurate, clinically practical system for spatial vectorcardiography. Circulation, 13(5):737-749, 1956.

[45] V. Fuster, L. E. Ryden, D. S. Cannom, H. J. Crijns, A. B. Curtis, K. A. Ellenbogen, J. L. Halperin, J. Y. Le Heuzey, G. N. Kay, J. E. Lowe, S. B. Olsson, E. N. Prystowsky, J. L. Tamargo, S. Wann, S. C. Jr. Smith, A. K. Jacobs, C. D. Adams, J. L. Anderson, E. M. Antman, S. A. Hunt, R. Nishimura, J. P. Ornato, R. L. Page, B. Riegel, S. G. Priori, J. J. Blanc, A. Budaj, A. J. Camm, V. Dean, J. W. Deckers, C. Despres, K. Dickstein, J. Lekakis, K. McGregor, M. Metra, J. Morais, A. Osterspey, and J. L. Zamorano. ACC/AHA/ESC 2006 Guidelines for the management of patients with atrial fibrillation: a report of the American College of Cardiology/American Heart Association Task Force on Practice Guidelines and the European Society of Cardiology Committee for Practice Guidelines (Writing committee to revise the 2001 guidelines for the management of patients with atrial fibrillation): developed in collaboration with the European Heart Rhythm Association and the Heart Rhythm society. Circulation, 114(7):e257-e354, Aug 2006.

[46] E. P. Gerstenfeld, A. V. Sahakian, and S. Swiryn. Evidence for transient linking of atrial excitation during atrial fibrillation in humans. Circulation, 86(2):375-382, Aug 1992.

[47] M. S. Guillem, A. Bollmann, A. M. Climent, D. Husser, J. Millet, and F. Castells. How many leads are necessary for a reliable reconstruction of surface potentials during atrial fibrillation? IEEE Transactions on Information Technology in Biomedicine, (Under revision), 2008.

[48] M. S. Guillem, R. Brugada, B. Thibault, A.M. Climent, and J. Millet. Analysis of body surface maps in cardiac resynchronization therapy. Computers in Cardiology, (Bologna, Italy), 2008.

[49] M. S. Guillem, F. Castells, A. M. Climent, V. Bodi, F. J. Chorro, and J. Millet. Evaluation of lead selection methods for optimal reconstruction of body surface potentials. Journal of Electrocardiology, 41(1):26-34, Jan 2008.

[50] M. S. Guillem, F. Castells, A.M. Climent, F.J. Chorro, V. Bodi, and J. Millet. Lead selection methods from multilead electrocardiographic 
recordings. Annual ISCE Conference (International Society for Computerized Electrocardiology), (Cancun, Mexico), 2007.

[51] M. S. Guillem, A. M. Climent, A. Bollmann, D. Husser, J. Millet, and F. Castells. Limitations of Dower's inverse transform for the study of atrial loops during atrial fibrillation. PACE, (Under revision), 2008.

[52] M. S. Guillem, A. M. Climent, F. Castells, D. Husser, J. Millet, A. Arya, C. Piorkowski, and A. Bollmann. Non-invasive mapping of human atrial fibrillation. Journal of Cardiovascular Electrophysiology, (Accepted for publication), 2008 .

[53] M. S. Guillem, A.M. Climent, A. Bollmann, D. Husser, J. Millet, and F. Castells. Can orthogonal leads be derived from the standard electrocardiogram during atrial fibrillation? European Congress for Medical and Biomedical Engineering, (Antwerp, Belgium), 2008.

[54] M. S. Guillem, A.M. Climent, A. Bollmann, D. Husser, J. Millet, and F. Castells. Performance of reduced lead systems in the study of atrial fibrillation. Biosignal Conference, (Brno, Czech Republic), 2008.

[55] M. S. Guillem, A.M. Climent, D. Husser, J. Millet, and A. Bollmann. Noninvasive, high-density mapping of human atrial fibrillation - Introduction and illustration of a novel diagnostic tool. Computers in Cardiology, (Durham, EE.UU.), 2007.

[56] M. S. Guillem, J. Millet, and F. J. Chorro. Dispositivo portaelectrodos para la aplicacion de una pluralidad de electrodos al torso de una persona, y conjunto que comprende la pluralidad de tales dispositivos. Patent number:ES2264905, Jan 2008.

[57] M. S. Guillem, A. V. Sahakian, and S. Swiryn. Derivation of orthogonal leads from the 12-lead ECG: accuracy of a single transform. Computers in Cardiology, (Valencia, Spain), 2006.

[58] M. S. Guillem, A. V. Sahakian, and S. Swiryn. Derivation of orthogonal leads from the 12-lead electrocardiogram. Performance of an atrial-based transform for the derivation of $\mathrm{P}$ loops. Journal of Electrocardiology, 41(1):19-25, Jan 2008.

[59] M. Haissaguerre, P. Jais, D. C. Shah, A. Takahashi, M. Hocini, G. Quiniou, S. Garrigue, A. Le Mouroux, P. Le Metayer, and J. Clementy. Spontaneous initiation of atrial fibrillation by ectopic beats originating in the pulmonary veins. New England Journal of Medicine, 339(10):659-666, Sept 1998. 
[60] K. Hisamatsu, K. F. Kusano, H. Morita, S. Takenaka, S. Nagase, K. Nakamura, T. Emori, H. Matsubara, and T. Ohe. Usefulness of body surface mapping to differentiate patients with Brugada syndrome from patients with asymptomatic Brugada syndrome. Acta Medica Okayama, 58(1):29-35, Feb 2004.

[61] R. Hoekema, G. Uijen, and Oosterom A. van. The number of independent signals in body surface maps. Methods of Information in Medicine, 38(2):119-124, Jun 1999.

[62] R. Hoekema, G. J. Uijen, and A. van Oosterom. On selecting a body surface mapping procedure. Journal of Electrocardiology, 32(2):93-101, Apr 1999.

[63] M. Holm, S. Pehrson, M. Ingemansson, L. Sörnmo, R. Johansson, L. Sandhall, M. Sunemark, B. Smideberg, C. Olsson, and S. B. Olsson. Non-invasive assessment of the atrial cycle length during atrial fibrillation in man: introducing, validating and illustrating a new ECG method. Cardiovascular Research, 38(1):69-81, 1998.

[64] L. G. Horan, N. C. Flowers, and D. A. Brody. Principal factor waveforms of the thoracic QRS complex. Circulation Research, 15:131-145, August 1964.

[65] R. P. M. Houben and M. A. Allessie. Processing of intracardiac electrograms in atrial fibrillation - Diagnosis of electropathological substrate of AF. IEEE Engineering in Medicine and Biology Magazine, 25(6):40-51, Nov 2006.

[66] D. Husser, M. Stridh, L. Sörnmo, I. Toepffer, H. U. Klein, S. B. Olsson, and A. Bollmann. Electroatriography - Time-frequency analysis of atrial fibrillation from modified 12-lead ECG configurations for improved diagnosis and therapy. Medical Hypotheses, 68(3):568-573, 2007.

[67] Z. Ihara, Oosterom A. van, V. Jacquemet, and R. Hoekema. Adaptation of the standard 12-lead electrocardiogram system dedicated to the analysis of atrial fibrillation. Journal of Electrocardiology, 40(1):68-68, Jan 2007.

[68] J. Jalife. Rotors and spiral waves in atrial fibrillation. Journal of Cardiovascular Electrophysiology, 14(7):776-780, Jul 2003.

[69] J. Jalife, O. Berenfeld, and M. Mansour. Mother rotors and fibrillatory conduction: a mechanism of atrial fibrillation. Cardiovascular Research, 54(2):204-216, May 2002.

[70] A. Kadish, J. Hauck, B. Pederson, G. Beatty, and C. Gornick. Mapping of atrial activation with a noncontact, multielectrode catheter in dogs. Circulation, 99(14):1906-1913, 1999. 
[71] K. T. S. Konings, C. J. H. J. Kirchhof, J. R. L. M. Smeets, H. J. J. Wellens, O. C. Penn, and M. A. Allessie. High-density mapping of electricallyinduced atrial-fibrillation in humans. Circulation, 89(4):1665-1680, Apr 1994.

[72] F. Kornreich and D. Brismee. The missing waveform information in the orthogonal electrocardiogram (Frank leads). ii. diagnosis of left ventricular hypertrophy and myocardial infarction from "total" surface waveform information. Circulation, 48(5):996-1004, Nov 1973.

[73] F. Kornreich, T. J. Montague, M. Kavadias, J. Segers, P. M. Rautaharju, M. B. Horacek, and B. Taccardi. Qualitative and quantitative analysis of characteristic body surface potential map features in anterior and inferior myocardial infarction. American Journal of Cardiology, 60(16):1230-1238, Dec 1987.

[74] F. Kornreich, T. J. Montague, and P. M. Rautaharju. Identification of first acute $\mathrm{Q}$ wave and non-Q wave myocardial infarction by multivariate analysis of body surface potential maps. Circulation, 84(6):2442-2453, Dec 1991.

[75] F. Kornreich, T. J. Montague, and PM. Rautaharju. Body surface potential mapping of ST segment changes in acute myocardial infarction. Implications for ECG enrollment criteria for thrombolytic therapy. Circulation, 87(3):773-782, 1993.

[76] F. Kornreich, T. J. Montague, G. van Herpen, P. M. Rautaharju, P. Smets, and M. Dramaix. Improved prediction of left ventricular mass by regression analysis of body surface potential maps. American Journal of Cardiology, 66(4):485-492, Aug 1990.

[77] J. A. Kors, Herpen G. van, A. C. Sittig, and J. H. van Bemmel. Reconstruction of the frank vectorcardiogram from standard electrocardiographic leads: diagnostic comparison of different methods. European Heart Journal, 11(12):1083-1092, Dec 1990.

[78] J. A. Kors and G. van Herpen. How many electrodes and where?: A poldermodel for electrocardiography. Journal of Electrocardiology, 35 Suppl:7-12, 2002.

[79] P. Langley, J.J. Rieta, M. Stridh, J. Millet, L. Sörnmo, and A. Murray. Comparison of atrial signal extraction algorithms in 12-lead ECGs with atrial fibrillation. IEEE Transactions on Biomedical Engineering, 53(2):343-346, Feb. 2006.

[80] S. Lazar, S. Dixit, F. E. Marchlinski, D. J. Callans, and E. P. Gerstenfeld. Presence of left-to-right atrial frequency gradient in paroxysmal but not 
persistent atrial fibrillation in humans. Circulation, 110(20):3181-3186, 2004 .

[81] Ch. L. Levkov. Orthogonal electrocardiogram derived from the limb and chest electrodes of the conventional 12-lead system. Medical $\&$ Biological Engineering \& Computing, 25:155-164, 1987.

[82] T. Lewis. Auricular fibrillation and its relationship to clinical irregularity of the heart. British Heart Journal, 1:306-372, 1910.

[83] L. S. Lilly. Pathophysiology of heart disease. A collaborative study of medical students and faculty. Lippincott Williams and Wilkins, Baltimore, Maryland, USA, 3 edition, 2003.

[84] R. L. Lux. Electrocardiographic body surface potential mapping. Crit Reviews in Biomedical Engineering, 8(3):253-279, 1982.

[85] R. L. Lux, M. J. Burgess, R. F. Wyatt, A. K. Evans, G. M. Vincent, and J. A. Abildskov. Clinically practical lead systems for improved electrocardiography: comparison with precordial grids and conventional lead systems. Circulation, 59(2):356-363, Feb 1979.

[86] R. L. Lux and R. Greg. New leads for P wave detection and arrhythmia classification. Journal of Electrocardiology, 37(4 Pt 2):80, Oct 2004.

[87] R. L. Lux and F. Kornreich. Crossroads in electrocardiographic lead development: a roadmap to the future of electrocardiographic leads in clinical electrocardiology. Journal of Electrocardiology, 41(3):183-186, 2008 .

[88] R. L. Lux, C. R. Smith, R. F. Wyatt, and J. A. Abildskov. Limited lead selection for estimation of body surface potential maps in electrocardiography. IEEE Transactions on Biomedical Engineering, 25(3):270-276, May 1978.

[89] J. E. Madias. Comparison of $\mathrm{P}$ waves recorded on the standard electrocardiogram, the Lewis lead, and saline-filled central venous catheterbased intracardiac electrocardiogram. American Journal of Cardiology, 94(4):474-478, 2004.

[90] J. Malmivuo and R. Plonsey. Bioelectromagnetism. Principles and applications of bioelectric and biomagnetic fields. Oxford University Press, New York, 1995.

[91] R. Mandapati, A. Skanes, J. Chen, O. Berenfeld, and J. Jalife. Stable microreentrant sources as a mechanism of atrial fibrillation in the isolated sheep heart. Circulation, 101(2):194-199, Jan 2000. 
[92] M. Mansour. Highest dominant frequencies in atrial fibrillation: a new target for ablation? J.Am.Coll.Cardiol., 47(7):1408-1409, Apr 2006.

[93] M. Mansour, R. Mandapati, O. Berenfeld, J. Chen, F. H. Samie, and J. Jalife. Left-to-right gradient of atrial frequencies during acute atrial fibrillation in the isolated sheep heart. Circulation, 103(21):2631-2636, May 2001.

[94] S. J. Maynard, I. B. Menown, G. Manoharan, J. Allen, Anderson J. McC, and A. A. Adgey. Body surface mapping improves early diagnosis of acute myocardial infarction in patients with chest pain and left bundle branch block. Heart, 89(9):998-1002, Sep 2003.

[95] A. J. McClelland, C. G. Owens, I. B. Menown, M. Lown, and A. A. Adgey. Comparison of the 80-lead body surface map to physician and to 12-lead electrocardiogram in detection of acute myocardial infarction. American Journal of Cardiology, 92(3):252-257, Aug 2003.

[96] M. Medvegy, I. Preda, P. Savard, A. Pinter, G. Tremblay, J. B. Nasmith, D. Palisaitis, and R. A. Nadeau. New body surface isopotential map evaluation method to detect minor potential losses in non-Q-wave myocardial infarction. Circulation, 101(10):1115-1121, Mar 2000.

[97] I. B. Menown, G. Mackenzie, and A. A. Adgey. Optimizing the initial 12-lead electrocardiographic diagnosis of acute myocardial infarction. European Heart Journal, 21(4):275-283, Feb 2000.

[98] G. K. Moe and J. A. Abildskov. Atrial fibrillation as a self-sustaining arrhythmia independent of focal discharge. American Heart Journal, 58(1):59-70, 1959 .

[99] T. J. Montague, D. E. Johnstone, C. A. Spencer, L. D. Lalonde, M. J. Gardner, M. G. O'Reilly, and B. M. Horacek. Non-q-wave acute myocardial infarction: body surface potential map and ventriculographic patterns. American Journal of Cardiology, 58(13):1173-1180, Dec 1986.

[100] S. Nattel. New ideas about atrial fibrillation 50 years on. Nature, 415(6868):219-216, Jan 2002.

[101] J. Ng, A. V. Sahakian, W. G. Fisher, and S. Swiryn. Atrial flutter vector loops derived from the surface ECG: does the plane of the loop correspond anatomically to the macroreentrant circuit? Journal of Electrocardiology, 36 Suppl:181-186, 2003.

[102] J. Ng, A. V. Sahakian, W. G. Fisher, and S. Swiryn. Surface ECG vector characteristics of organized and disorganized atrial activity during atrial fibrillation. Journal of Electrocardiology, 37 Suppl:91-97, 2004. 
[103] C. G. Owens, A. J. McClelland, S. J. Walsh, B. A. Smith, A. Tomlin, J. W. Riddell, M. Stevenson, and A. A. Adgey. Prehospital 80-lad mapping: Does it add significantly to the diagnosis of acute coronary syndromes? Journal of Electrocardiology, 37(4 Pt 2):223-232, Oct 2004.

[104] J. Pan and W. J. Tompkins. A real-time QRS detection algorithm. IEEE Transactions on Biomedical Engineering, 32(3):230-236, 1985.

[105] S. Petrutiu, G. M. Nijm, H. Al Angari, S. Swiryn, and A. V. Sahakian. Atrial fibrillation and waveform characterization - a time domain perspective in the surface ECG. IEEE Engineering in Medicine and Biology Magazine, 25(6):24-30, Nov 2006.

[106] U. Richter, M. Stridh, A. Bollmann, D. Husser, and L. Sörnmo. Spatial characteristics of atrial fibrillation electrocardiograms. Journal of Electrocardiology, 41(2):165-172, Mar 2008.

[107] J.J. Rieta, F. Castells, C. Sanchez, V. Zarzoso, and J. Millet. Atrial activity extraction for atrial fibrillation analysis using blind source separation. IEEE Transactions on Biomedical Engineering, 51(7):11761186, July 2004.

[108] F. X. Roitinger, A. SippensGroenewegen, M. R. Karch, P. R. Steiner, W. S. Ellis, and M. D. Lesh. Organized activation during atrial fibrillation in man. Journal of Cardiovascular Electrophysiology, 9(5):451-461, 1998.

[109] KM Ropella, AV Sahakian, JM Baerman, and S Swiryn. The coherence spectrum. A quantitative discriminator of fibrillatory and nonfibrillatory cardiac rhythms. Circulation, 80(1):112-119, 1989.

[110] P. Rubel, I. Benhadid, and J. Fayn. Quantitative assessment of eight different methods for synthesizing Frank VCGs from simultaneously recorded standard ecg leads. Journal of Electrocardiology, 24 Supp:197202,1992 .

[111] S. Sangwatanaroj, S. Prechawat, B. Sunsaneewitayakul, S. Sitthisook, P. Tosukhowong, and K. Tungsanga. New electrocardiographic leads and the procainamide test for the detection of the Brugada sign in sudden unexplained death syndrome survivors and their relatives. European Heart Journal, 22(24):2290-2296, Dec 2001.

[112] W. Shimizu, K. Matsuo, M. Takagi, Y. Tanabe, T. Aiba, A. Taguchi, K. Suyama, T. Kurita, N. Aihara, and S. Kamakura. Body surface distribution and response to drugs of ST segment elevation in Brugada syndrome: clinical implication of eighty-seven-lead body surface potential mapping and its application to twelve-lead electrocardiograms. Journal of Cardiovascular Electrophysiology, 11:396-404, 2000. 
[113] H. J. Sih, D. P. Zipes, E. J. Berbari, and J. E. Olgin. A high-temporal resolution algorithm for quantifying organization during atrial fibrillation. IEEE Transactions on Biomedical Engineering, 46(4):440-450, Apr 1999.

[114] D. U. Silverthorn. Human Physiology. An integrated approach. Benjamin Cummings, Pearson education, San Francisco, California, USA, 3 edition, 2004 .

[115] A. SippensGroenewegen, M. D. Lesh, F. X. Roithinger, W. S. Ellis, P. R. Steiner, L. A. Saxon, R. J. Lee, and M. M. Scheinman. Body surface mapping of counterclockwise and clockwise typical atrial flutter: a comparative analysis with endocardial activation sequence mapping. Journal of the American College of Cardiology, 35(5):1276-1287, Apr 2000.

[116] A. SippensGroenewegen, A. Natale, N. F. Marrouche, D. Bash, and J. Cheng. Potential role of body surface ECG mapping for localization of atrial fibrillation trigger sites. Journal of Electrocardiology, 37 Suppl:47$52,2004$.

[117] A. SippensGroenewegen, H. A. Peeters, E. R. Jessurun, A. C. Linnenbank, E. O. Robles de Medina, M. D. Lesh, and N. M. van Hemel. Body surface mapping during pacing at multiple sites in the human atrium: P-wave morphology of ectopic right atrial activation. Circulation, 97(4):369-380, Feb 1998.

[118] A. SippensGroenewegen, F. X. Roithinger, H. A. Peeters, A. C. Linnenbank, N. M. van Hemel, P. R. Steiner, and M. D. Lesh. Body surface mapping of atrial arrhythmias: atlas of paced $\mathrm{P}$ wave integral maps to localize the focal origin of right atrial tachycardia. Journal of Electrocardiology, 31 Suppl:85-91, 1998.

[119] A. C. Skanes, R. Mandapati, O. Berenfeld, J. M. Davidenko, and J. Jalife. Spatiotemporal periodicity during atrial fibrillation in the isolated sheep heart. Circulation, 98(12):1236-1248, Sep 1998.

[120] J. Slocum, A. V. Sahakian, and S. Swiryn. Diagnosis of atrial fibrillation from surface electrocardiograms based on computer-detected atrial activity. Journal of Electrocardiology, 25(1):1-8, 1992.

[121] L. Sörnmo and P. Laguna. Bioelectrical signal processing in cardiac and neurological applications, volume 1, chapter The electrocardiogram - A brief background, pages 411-452. Elsevier Academic Press, Amsterdam, The Netherlands, 2005.

[122] M. Stridh, A. Bollmann, S. B. Olsson, and L. Sörnmo. Detection and feature extraction of atrial tachyarrhythmias. IEEE Engineering in Medicine and Biology Magazine, 25(6):31-39, Nov.-Dec. 2006. 
[123] M. Stridh and L. Sörnmo. Spatiotemporal qrst cancellation techniques for analysis of atrial fibrillation. IEEE Transactions on Biomedical Engineering, 48(1):105-111, Jan 2001.

[124] M. Stridh, L. Sörnmo, C. J. Meurling, and S. B. Olsson. Characterization of atrial fibrillation using the surface ECG: time-dependent spectral properties. IEEE Transactions on Biomedical Engineering, 48(1):19-27, Jan 2001.

[125] M. Stridh, L. Sörnmo, C. J. Meurling, and S.B. Olsson. Sequential characterization of atrial tachyarrhythmias based on ECG time-frequency analysis. IEEE Transactions on Biomedical Engineering, 51(1):100-114, Jan. 2004.

[126] B. Taccardi. Distribution of heart potentials on the thoracic surface of normal human subjects. Circulation Research, 12:341-352, 1963.

[127] B. Taccardi, B. B. Punske, R. L. Lux, R. S. MacLeod, P. R. Ershler, T. J. Dustman, and Y. Vyhmeister. Useful lessons from body surface mapping. Journal of Cardiovascular Electrophysiology, 9(7):773-786, Jul 1998.

[128] M. Takagi, I. Toda, K. Takeuchi, and J. Yoshikawa. Utility of right precordial leads at higher intercostal space positions to diagnose Brugada syndrome. Pacing and Clinical Electrophysiology, 25(2):241-242, Feb 2002 .

[129] I. Tonooka, I. Kubota, Y. Watanabe, K. Tsuiki, and Yasui S. Isointegral analysis of Body Surface Maps for the assessment of location and size of myocardial infarction. American Journal of Cardiology, 52:1174-1180, Dec 1983.

[130] G. J. Uijen and A. van Oosterom. The performance of informationtheoretic criteria in detecting the number of independent signals in multilead ECGs. Methods of Information in Medicine, 31(4):256-262, Nov 1992.

[131] A. van Oosterom, Z. Ihara, V. Jacquemet, and R. Hoekema. Vectorcardiographic lead systems for the characterization of atrial fibrillation. Journal of Electrocardiology, 40(4):343.e1-343.e11, Oct 2006.

[132] A. Vulpian. Note sur les effets de la faradisation directe des ventricules du coeur chez le chien. Archives de Physiologie Normale et Pathologique, 6:975-980, 1874 .

[133] A. Waller. On the electromotive changes connected with the beat of the mammalian heart and of the human heart in particular. Philos Trans $R$ Soc, B180:169-194, 1889. 
[134] W. A. Wattigney, G. A. Mensah, and J. B. Croft. Increasing trends in hospitalization for atrial fibrillation in the united states, 1985 through 1999. Circulation, 108:711-716, 2003.

[135] F. N. Wilson, A. M. Macleod, P. S. Barker, and F. D. Johnston. The determination and the significance of the areas of the ventricular deflections of the electrocardiogram. American Heart Journal, 10:46-61, 1934.

[136] H. K. Wolf, P. M. Rautaharju, V. C. Unite, and J. Stewart. Evaluation of synthesized standard 12-leads and frank vector leads. Advances in Cardiology., 16:87-97, 1976.

[137] D. G. Wyse and B. J. Gersh. Atrial fibrillation: a perspective. thinking inside and outside the box. Circulation, 109:3089-3095, 2004. 